NOTE TO USERS

This reproduction is the best copy available.

(B)

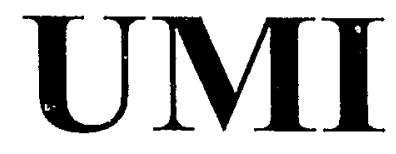

Reproduced with permission of the copyright owner. Further reproduction prohibited without permission. 
Reproduced with permission of the copyright owner. Further reproduction prohibited without permission. 


\title{
A COMPUTATIONAL STUDY OF STRUCTURE DEVELOPMENT AND TEXTURE FORMATION IN CARBONACEOUS MESOPHASE FIBERS
}

By

\author{
Shujuan Hong, B. Eng. \\ Tianjin University, Tianjin, China, 1989
}

A thesis presented to Ryerson University in partial fulfillment of the requirements for the degree of Master of Applied Science in the Program of

Chemical Engineering

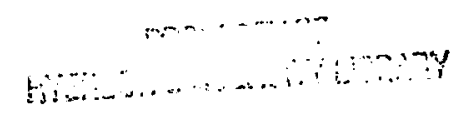

Toronto, Ontario, Canada, 2004

(C) Shujuan Hong, 2004 
UMI Number: EC52924

\section{INFORMATION TO USERS}

The quality of this reproduction is dependent upon the quality of the copy submitted. Brol en or indistinct print, colored or poor quality illustrations and photographs, print bleed-through, substandard margins, and improper alignment can adversely affect reproduction.

In the unlikely event that the author did not send a complete manuscript and there are missing pages, these will be noted. Also, if unauthorized copyright material had to be removed, a note will indicate the deletion.

(B)

\section{UMI}

UMI Microform EC52924

Copyright 2008 by ProQuest LLC.

All rights reserved. This microform edition is protected against unauthorized copying under Title 17, United States Code.

ProQuest LLC

789 E. Eisenhower Parkway

PO Box 1346

Ann Arbor, MI 48106-1346 


\section{Borrower's Page}

Ryerson University requires the signatures of all persons using or photocopying this thesis. Please sign below, and give address and date. 


\title{
Abstract
}

A Computational Study of Structure Development and Texture Formation in Carbonaceous Mesophase Fibers

\author{
Master of Applied Science
}

2004 Shujuan Hong

\author{
Chemical Engineering \\ Ryerson University
}

In this thesis, thermal relaxation phenomena after the melt-extrusion of a rigid discotic uniaxial nematic mesophase pitch were studied using mathematical modeling and computer simulation. The Ericksen and Landau-de Gennes continuum theories were used to investigate the structure development and texture formation across mesophase pitch based carbon fibers.

It is found that during the thermal relaxation, discotic nematic molecules stored elastic free energy decays. The distorted nematic molecular profile reoriented to release the stored elastic free energy. The difference in time scales for molecular reorientation and thermal relaxation resulted in different transverse textures. The rate at which the fibers are cooled is the main factor in controlling the structure development. A slow cooling rate would permit nematic discotic molecules to reorient to a well developed (radial or onion) texture. The random texture is a result of rapid quenching. The numerical results are consistent with published experimental observations. 


\section{Acknowledgements}

I would like to express my gratitude to my supervisor, Philip Chan, for his continuous guidance, advice, and inspiration throughout my graduate study.

A note of special thanks also gives to my committee, for their helpful discussions and encouragement.

Financial support from Chemical Engineering Department of Ryerson University is also acknowledged.

Finally, I would like to thank my family for their love, understanding and support throughout my life-long learning. 


\section{Table of Contents}

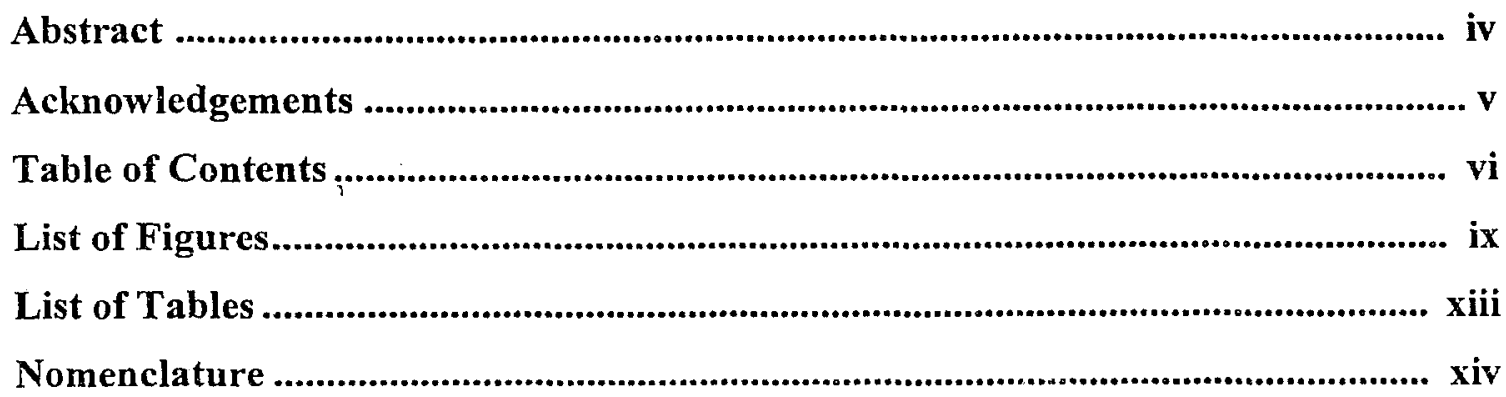

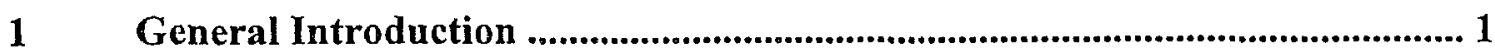

1.1 Introduction to Carbon Fibers ........................................................... 1

1.2 Mesophase Pitch Based Carbon Fibers .................................................... 4

1.3 Mesophase Pitch Based Carbon Fibers Structure Formation ..................... 4

1.3.1 Mode Selection.................................................................. 5

1.3.2 Structure Development....................................................... 5

1.3.3 Structure and Properties Correlation .................................... 5

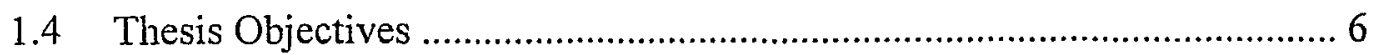

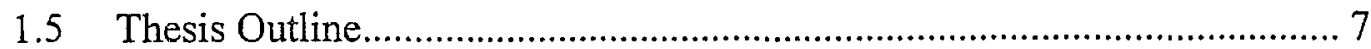

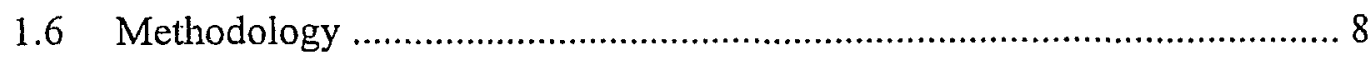

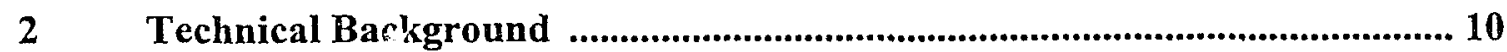

2.1 Liquid Crystal Basic Concepts........................................................ 10

2.1.1 Definition of Liquid Crystal Phases (Mesophases) .................. 10

2.1.2 Liquid Crystal Classification .............................................. 11

2.1.3 Orientational Order .......................................................... 12

2.2 Frank-Oseen-Zocher Static Elastic Continuum Theory ....................... 15

2.3 Leslie-Ericksen Continuum Theory …............................................... 17

2.3.1 Mass Balance .................................................................. 18

2.3.2 Linear Momentum Balance.................................................... 18

2.3.3 Angular Momentum Balance ............................................... 19

2.4 Landau-de Gennes Nematic Continuum Theory ................................ 21 
2.5 Ericksen Continuum Theory for Nonhomogenously Ordered Nematic

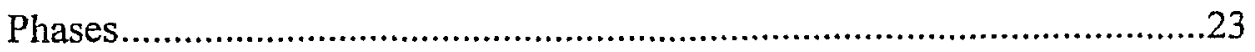

2.6 Nematic Potential and Equilibrium Orientational Order …….....................24

$3 \quad$ Literature Review ............................................................................................. 27

3.1 Introduction to Mesophase Pitch ……………………………………..... 27

3.2 Melt Spinning and Process Effect on Fibers Structures and Properties..... 29

3.2.1 Melt Spinning Process and Process Parameters ......................... 30

3.2.2 Process Variable Dependent Textures ......................................... 31

3.2.3 Different Textures Observed from The Experimental Work........ 32

3.2.4 Texture Dependent Properties....................................................... 33

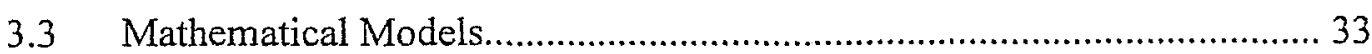

3.3.1 Wang and Rey's Elastic Model................................................... 34

3.3.2 McHugh and Edie's Model...................................................... 36

4 Gne-Dimensional Model Development, Results and Discussion ...................... 39

4.1 Introduction to One-Dimensional Model .................................................. 39

4.2 Balance Equation Formulation ............................................................... 42

4.2.1 Landau-de Gennes Free Energy .................................................. 42

4.2.2 Angular Momentum Balance Equation and Scalar Order Parameter Balance Equation ......................................................... 43

4.2.3 Dimensionless Equations ........................................................... 45

4.2.4 Auxiliary Equations ................................................................... 48

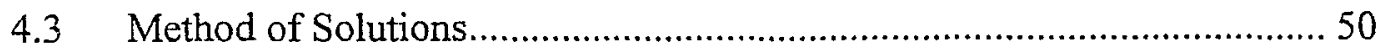

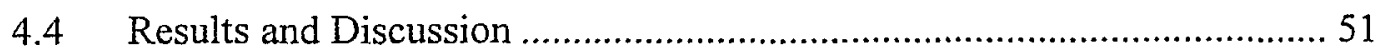

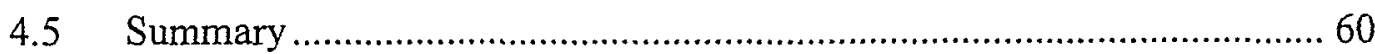

5 Two-Dimensional Model Development, Results and Discussion..................... 61

5.1 Introduction to Two-Dimensional Model .................................................. 63

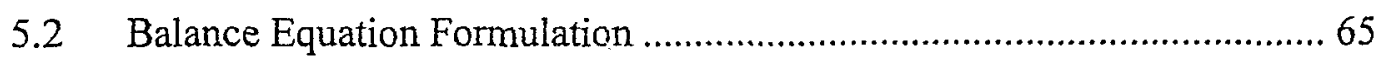

5.2.1 Landau de-Gennes Free Energy ................................................. 65 


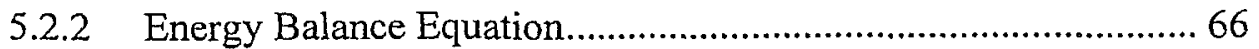

5.2.3 Angular Momentum and Scalar Order Parameter Balance

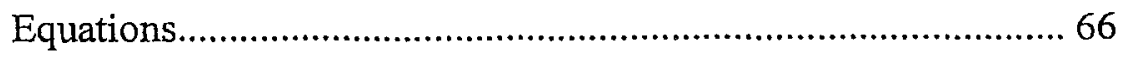

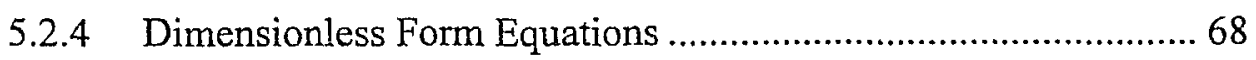

5.3 Auxiliary Equations ........................................................................ 70

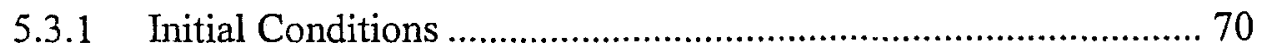

5.3.2 Boundary Conditions ................................................................ 71

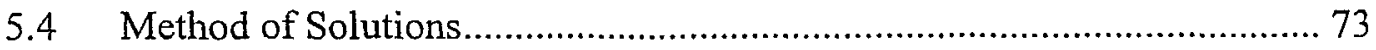

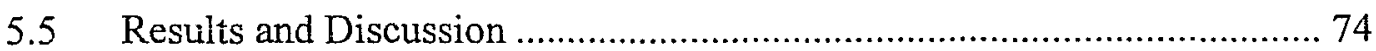

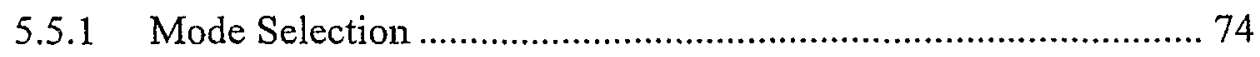

5.5.2 Structure Development ................................................................ 75

5.5.3 Time Evolution of Frank Elastic Free Energy $F_{\mathrm{d}}{ }^{*}$..................... 78

5.5.4 Temperature and The Scalar Order Parameter Profiles................ 80

5.5.5 Time Evolution of Molecular Free Energy $F_{\mathrm{S}}{ }^{*}$............................ 85

5.5.6 Effect of $\alpha$ on The Thermal Relaxation..................................... 87

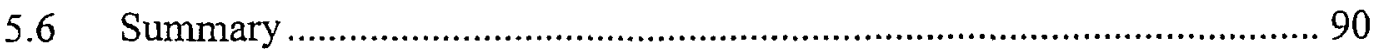

6 Conclusions and Recommendations for Future Work ..................................91

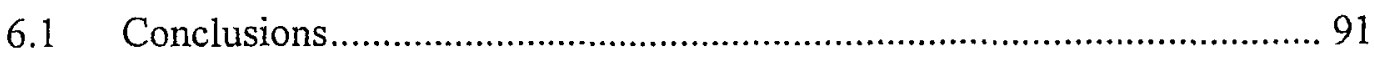

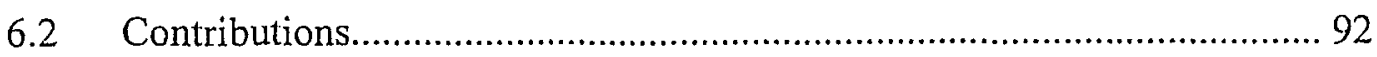

6.3 Recommendations for Future Work......................................................... 93

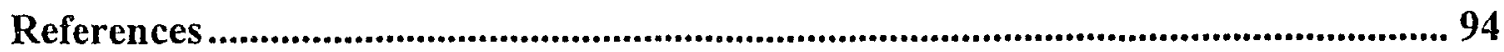

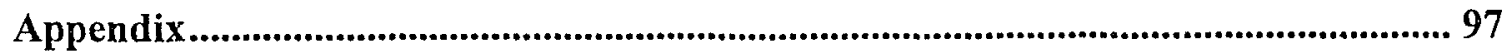




\section{List of Figures}

Figure 2.1 The liquid crystal phases: (a) Nematic, (b) Cholesteric and (c) Smectic-A.. 12

Figure 2.2 Schematic representation of molecular director in (a) rod-like nematic and (b) discotic nematic phases ............................................................................ 12

Figure 2.3 Schematic representation of orientation angle $\theta$ in (a) rod-like nematic and (b) discotic nematic ....................................................................................... 13

Figure 2.4 Typical variations of the scalar order parameter $S$ for (a) thermotropic liquid crystals with temperature $T$ and (b) lyotropic liquid crystals with concentration $c . T_{\mathrm{NI}}$ and $c_{\mathrm{NI}}$ are the first order transition points 14

Figure 2.5 Three types of deformation in nematics: (a) rod-like molecules (b) discotic molecules. $K_{1}$-splay deformation, $K_{2}$-twist deformation and $K_{3}$-bend deformation 16

Figure 2.6 Typical dependency of the free energy on the scalar order parameter $S$. For $U<8 / 3$, the stable phase is isotropic; $8 / 3<U<3$, nematic and isotropic liquid phase coexist; $U>3$, the system always approaches the nematic state

Figure 3.1 Typical poly-nuclear aromatic hydrocarbons in mesophase produced from coal and petroleum 28

Figure 3.2 The dependency of viscosity on temperature for mesophase pitch, isotropic pitch and nylon- 6

Figure 3.3 Melt spinning process and process parameters 31 
Figure 3.4 Temperature variation in elastic constants and transverse textures predicted by Wang and Rey. The temperature is scaled by the transition temperature $T_{\mathrm{N} 1}$ at which $K=K_{3}=K_{1}$, and the elastic constants are scaled by $K$. 35

Figure 3.5 McHugh and Edie's model results. It shows that the round shape spinnerets generate a radial texture fiber; the non-circular cross sectional shape spinnerets will create a fiber with linear texture, which would have higher degree of orientation 37

Figure 4.1 Schematic representation of the disc-like molecules within a section of a carbonaceous mesophase fiber, and the definition of the Cartesian coordinate system. $\theta$ is the planar director orientation angle measured in radians, and $R$ is the radius of the fiber 41

Figure 4.2 Typical relaxation phenomena for molecular orientation for the case $\alpha=10^{2}$ and $\beta=10^{4}$, in fiber radial direction at the following dimensionless times $t^{*}$ : (a) 0.0 , (b) 0.001 , (c) 0.002 , (d) 0.006 , and (e) 0.05 . 52

Figure 4.3 Time evolution of the dimensionless Frank elastic free energy $F_{\mathrm{d}}^{*}$ for the case $\alpha=10^{2}$ and $\beta=10^{4} . \tau_{\mathrm{d}}$ and $\tau_{\mathrm{o}}$ are defined as the time required for the molecules to reorient such that the Frank elastic free energy $F_{\mathrm{d}}{ }^{*}$ decreases by $86.5 \%$ and $98 \%$, respectively 54

Figure 4.4 Typical relaxation phenomena for (a) the temperature profile and (b) the scalar order parameter profile in fiber radial direction for the case $\alpha=10^{2}$ and $\beta=10^{4}$ at the following dimensionless times $t^{*}: 0.0001$ (diamond), 0.001 (square), 0.005 (triangle), and 0.05 (circle). 56 
Figure 4.5 Time evolution of the dimensionless molecular free energy $F_{\mathrm{S}}^{*}$ for the case $\alpha=10^{2}$ and $\beta=10^{4} . \tau_{\alpha}$ is the time for the molecular free energy to reach steady state. 57

Figure 4.6 Thermal diffusivity $\alpha$ effect on the thermal relaxation. The dasined line represents the thermal relaxation time scale versus $\alpha$. The two solid lines represent the molecule reorientation time scales versus $\alpha . \alpha_{0}$ is defined as the cross point between $\tau_{0}$ and $\tau_{\alpha}$, and $\alpha_{\mathrm{d}}$ is the cross point between $\tau_{\mathrm{d}}$ and $\tau_{\alpha}$

Figure 5.1 Schematic representation of the disc-like molecules within a section of a carbonaceous mesophase fiber of radius $R$, and the definition of the coordinate systems. The polar coordinates $r-\varphi$ are expressed by dashed lines. The Cartesian coordinates $x-y$ are expressed by solid lines. $\theta$ is the planar director orientation angle measured in radians, which is the angle between director $\mathbf{n}$ and polar $r$ at point $(x, y)$ 64

Figure 5.2 Typical relaxation phenomena for molecular orientation for the case $\alpha=10^{3}$ and $\beta=10^{4}$, across fiber cross section at the following dimensionless times $t^{*}:$ (a) 0.0 , (b) 0.01 , (c) 0.016 , and (d) 0.05

Figure 5.3 Time evolution of the dimensionless Frank elastic free energy $F_{\mathrm{d}}^{*}$ for the case $\alpha=10^{3}$ and $\beta=10^{4} . \tau_{\mathrm{d}}$ and $\tau_{\mathrm{o}}$ are defined as the time required for the molecules to reorient such that the Frank elastic free energy $F_{\mathrm{d}}{ }^{*}$ decreases by $86.5 \%$ and $98 \%$, respectively. 79 
Figure 5.4 Typical relaxation phenomena of the temperature profile for the case $\alpha=10^{3}$ and $\beta=10^{4}$ at the following dimensionless times $t^{*}:$ (a) 0.02 , (b)

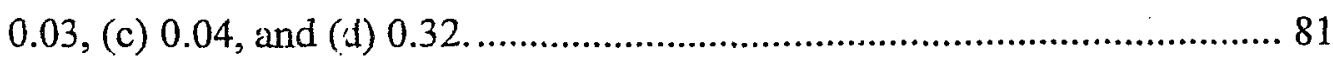

Figure 5.5 Typical relaxation phenomena of the scalar order parameter profile for the case $\alpha=10^{3}$ and $\beta=10^{4}$ at the following dimensionless times $t^{*}$ : (a) 0.02 , (b)

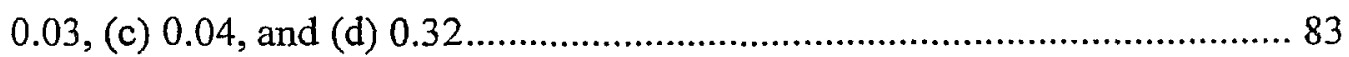

Figure 5.6 Time evolution of the dimensionless molecular free energy $F_{\mathrm{S}}^{*}$ for the case $\alpha=10^{3}$ and $\beta=10^{4} . \tau_{\alpha}$ is the time for the molecular free energy to reach steady state 86

Figure 5.7 The effect of the thermal diffusivity $\alpha$ on the thermal relaxation. The dashed line represents the thermal relaxation time scale versus $\alpha$. The two solid lines represent the molecule reorientation time scales versus $\alpha . \alpha_{0}$ is defined as the cross point between $\tau_{0}$ and $\tau_{\alpha}$, and $\alpha_{\mathrm{d}}$ is the cross point between $\tau_{\mathrm{d}}$ and $\tau_{\alpha}$ 89 


\section{List of Tables}

Table 1.1 Property comparisons between PAN and MP-based carbon fibers ................ 2

Table 1.2 Applications and characteristics of carbon fiber composites ....................... 3

Table 4.1 Elastic constants........................................................................... 47

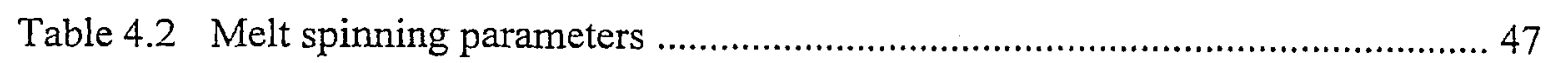

Table 4.3 Physical properties for carbon fibers ................................................. 47

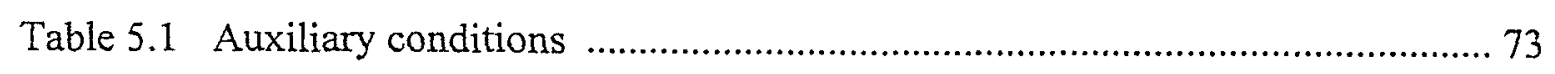




\section{Nomenclature}

\section{Roman Symbols}

A

A

B

$c$

$C_{p}$

$c_{\mathrm{NI}}$

C

$f_{0}(T)$

$f_{\mathrm{C}}$

$F_{\mathrm{C}}$

$F_{\mathrm{C}}{ }^{*}$

$f_{\mathrm{d}}$

$F_{\mathrm{d}}$

$F_{\mathrm{d}}{ }^{*}$

$f_{\mathrm{e}}$

$F_{\mathrm{e}}$

$F_{\mathrm{e}}^{*}$

$f_{\mathrm{L}}$

$F_{\mathrm{L}}$

$F_{i}{ }^{*}$

$f_{\mathrm{S}}$

$F_{\mathrm{S}}$

$F_{\mathrm{S}}{ }^{*}$

F

h

$\mathbf{h}_{\mathrm{B}}$
Landau-de Gennes free energy density coefficient

rate of deformation tensor

Landau-de Gennes free energy density coefficient

concentration

heat capacity

nematic-isotropic phase transition concentration

Landau-de Gennes free energy density coefficient

isotropic free energy density at temperature $T$

coupling elastic free energy density

coupling elastic free energy

dimensionless coupling elastic free energy

Frank elastic free energy density

Frank elastic free energy

dimensionless Frank elastic free energy

molecular elastic free energy density

molecular elastic free energy

dimensionless molecular elastic free energy

Landau-de Gennes free energy density

Landau-de Gennes free energy

dimensionless Landau-de Gennes free energy

molecular free energy density

molecular free energy

dimensionless molecular free energy

external body force per unit volume

the molecular field

bend component of $\mathbf{h}$ 


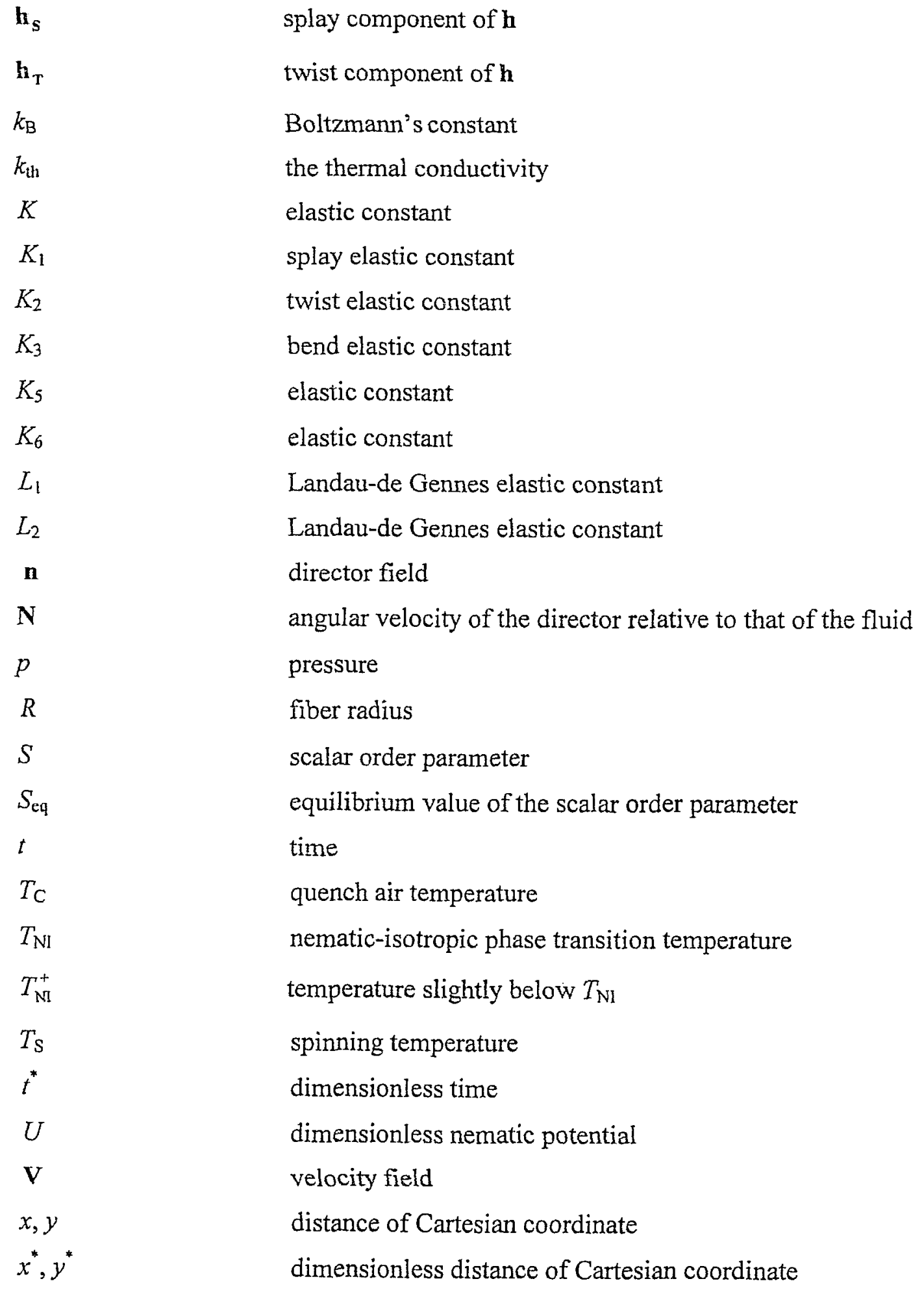




\section{Greek Symbols}

$\alpha$

$\alpha_{\mathrm{i}} \quad i=1, \ldots, 6$

$\alpha_{i}^{s} i=1, \ldots, 6$

$\beta$

$\beta_{\mathrm{i}}$

$\beta_{i}^{S}$

$\gamma_{1}$

$\gamma_{2}$

$\gamma_{1}^{S}$

$\gamma_{2}^{S}$

$\Gamma_{\mathrm{e}}$

$\Gamma_{v}$

$\theta$

$\kappa_{i}^{*}$

$\kappa_{i}$

$\pi$

$\rho$

$\Omega$

$v$

$\frac{\delta}{\delta(*)}$

\section{$\nabla$}

$\tau$

$\tau_{\alpha}$

$\tau_{d}$

$\tau_{0}$ dimensionless thermal diffusivity

Leslie viscosity coefficients

Leslie viscosities that depend on S

ratio of short range order elasticity to long range order elasticity

viscosities in equation (2.17), that not dependent on $S$

viscosities in equation (2.17), that dependent on $S$

rotational viscosity, that not dependent on $S$

irrotational viscosity, that not dependent on $S$

rotational viscosity that depend on $S$

irrotational viscosity that depend on $S$

elastic torque on the director per volume

viscous torque on the director per volume

director orientation angle

dimensionless elastic functions

elastic functions

pi

nematic density

vorticity tensor

disc concentration

functional derivative with respect to $\left(^{*}\right)$

gradient field

viscous stress tensor

time for the molecular free energy to reach steady state

time for the Frank free energy decreased by $86.5 \%$

time for the Frank free energy decreased by $98 \%$ 


\section{Chapter 1}

\section{General Introduction}

In this chapter, carbon fibers formation, structure and specific properties are briefly introduced. This is followed by a description of the thesis objectives, thesis outline and methodology of the thesis development.

\subsection{Introduction to Carbon Fibers}

Carbon fiber is a new class of advanced engineering material, which consists of 90 to 100 percent carbon content, with imperfect graphite crystalline or amorphous structure arranged along the fiber axis [1]. Because of their lightweight, high strength, high modulus and stiffness, carbon fibers are used in many high demand applications ranging from the aerospace industry to sporting goods. The excellent mechanical and transport properties of carbon fibers originate from the molecular structure.

The three most commonly used precursors to produce carbon fibers are viscous rayon, polyacrylonitrile (PAN) and petroleum or coal tar pitch $[1,2]$. The first commercialized rayon-based carbon fibers have been phased out due to the low yield and high cost of stabilization and high temperature heat treatment under high tension. Presently, the majority of carbon fiber is produced from PAN or mesophase pitch (MP) through filament spinning and post treatment processes.

PAN-based carbon fibers contain folded and interlinked fibril structure with no regular three-dimensional order [3]. The fibrillar structure gives this kind of carbon fiber high tensile and compressional strength. High strength PAN-based carbon fibers are usually used as hybrid composite with other materials, such as polymers, plastics, metals, and 
even cement. PAN-based carbon fiber reinforced composites are widely used in the main structure of military combat aircrafts, missiles, spacecraft and passenger airplanes.

In contrast, mesophase pitch based carbon fibers possess high degree of order both in the longitudinal and transverse directions of the fiber axis. The ordered structure gives mesophase pitch based carbon fibers high modulus and excellent transport properties such as thermal and electrical conductivities. High performance carbon fibers made from mesophase pitch have modulus up to $1000 \mathrm{GPa}$ and thermal conductivity 3 times that of copper [4]. High modulus mesophase pitch based carbon fibers are mainly used in engineering applications, such as the aerospace industry. Because of the high thermal conductivity and low thermal expansion, this class of carbon fibers is also being developed for applications where heat transfer is critical, such as brakes and engine rotating blades [5]. To provide some comparative information, Table 1.1 compares the properties of PAN based and mesophase pitch based carbon fibers, and Table 1.2 shows applications and characteristics of carbon fibers.

Table 1.1 Property comparisons between PAN and MP-based carbon fibers [3]

\begin{tabular}{lcc}
\multicolumn{1}{c}{ Property } & $\begin{array}{c}\text { PAN-based carbon } \\
\text { fibers }\end{array}$ & $\begin{array}{c}\text { Mesophase pitch-based carbon } \\
\text { fibers }\end{array}$ \\
\hline Tensile modulus, $\mathrm{GPa}$ & 290 & $690-965$ \\
Tensile strength, $\mathrm{MPa}$ & 5650 & 2410 \\
Elongation at break, \% & 1.8 & $0.4-0.27$ \\
Electrical resistance, $\mu \Omega \cdot \mathrm{cm}$ & 14.5 & 10 \\
Thermal conductivity, $\mathrm{W} / \mathrm{m} \cdot \mathrm{K}$ & 15 & $400-1100$ \\
Density, g/cm & & \\
Carbon content, $\%$ & 1.81 & 2.2 \\
Filament diameter, $10^{-6} \mathrm{~m}$ & 97 & 99 \\
Manufacturers & 11 & 10 \\
\hline
\end{tabular}


Table 1.2 Applications and characteristics of carbon fiber composites [3]

\begin{tabular}{|c|c|}
\hline Characteristics & Applications \\
\hline $\begin{array}{l}\text { High strength, specific toughness, } \\
\text { light weight }\end{array}$ & $\begin{array}{l}\text { Aerospace, road, train, and marine } \\
\text { transport, sporting goods }\end{array}$ \\
\hline $\begin{array}{l}\text { High dimensional stability, low } \\
\text { coefficient of thermal expansion and } \\
\text { low abrasion. }\end{array}$ & $\begin{array}{l}\text { Missiles, aircraft brakes, and aerospace } \\
\text { antenna }\end{array}$ \\
\hline $\begin{array}{l}\text { Good vibrational damping, strength, } \\
\text { and toughness }\end{array}$ & $\begin{array}{l}\text { Audio equipment, speakers of } \mathrm{Hi}-\mathrm{Fi} \\
\text { equipment, robotic arms }\end{array}$ \\
\hline Electrical conductivity & $\begin{array}{l}\text { Automobile hoods, casting and bases for } \\
\text { electronic equipment }\end{array}$ \\
\hline $\begin{array}{l}\text { Biological inertness and } x \text {-ray } \\
\text { permeability }\end{array}$ & $\begin{array}{l}\text { Surgery and } x \text {-ray equipment, implants, } \\
\text { medical applications in prostheses }\end{array}$ \\
\hline $\begin{array}{l}\text { Fatigue resistance, self-lubrication, } \\
\text { high damping }\end{array}$ & Textile machinery, general engineering \\
\hline $\begin{array}{l}\text { Chemical inertness, high corrosion } \\
\text { resistance }\end{array}$ & $\begin{array}{l}\text { Chemical industry, nuclear field valves, } \\
\text { seals }\end{array}$ \\
\hline Electromagnetic properties & $\begin{array}{l}\text { Large generator retaining } \\
\text { radiological equipment }\end{array}$ \\
\hline
\end{tabular}




\subsection{Mesophase Pitch Based Carbon Fibers}

Nowadays researchers and industry are more interested in the mesophase pitch based carbon fibers. Compared with PAN-based carbon fiber, mesophase pitch based carbon fibers have the following advantages: (1) Lower cost of raw materials $(\$ 0.25 / \mathrm{kg}$ comf red to $\$ 0.90 / \mathrm{kg}$ for PAN) [6]; (2) Higher yield (80\% compared to $40-45 \%$ for PAN) [2]; (3) Superior mechanical, thermal and electrical properties if produced properly [2]; and (4) The most important characteristic of the mesophase pitch material is its structure can be controlled by manipulating the material properties and melt spinning parameters. The process of producing mesophase pitch based carbon fibers begins with pitch being converted into liquid crystalline state through thermal treatment, then extruded using a melt spinning process followed by a series of post treatment specifically named as stabilization, carbonization and graphitization.

\subsection{Mesophase Pitch Based Carbon Fibers Structure Formation}

High performance mesophase pitch based carbon fibers consist of nematic discotic molecules that can be aligned in certain directions to form an ordered structure. Studies show that fiber structure is generated during the melt spinning process. Successful manufacturing of high performance carbon fiber from mesophase pitch depends on the control of texture formation, i.e., molecular orientation. The different orientations of the discotic molecules give rise to various ordered cross-sectional textures. The most commonly observed are random, zigzagged radial, radial, quasi-onion, and onion textures [1-3]. The structure formation is a comprehensive process involving mode (radial or onion) selection and the degree of molecule orientation evolution. 


\subsubsection{Mode Selection}

The mesophase pitch based carbon fibers structure formation results from the application of a series of extremely complex stress and thermal fields on a textured anisotropic viscoelastic material. Previous work [7-9] indicated that the mode selection between radial and onion is controlled by the spinning temperature, pitch structure and its anchoring behaviours, and the dimensions of the spinnerets. Mochida et al [10] and Ogale et al [11] observed that higher spinning temperature tend to form an onion texture; lower spinning temperature favors a radial texture. An intermediate temperature would produce fibers with a random texture. Matsumoto [12] and Hamada et al [13] formed the onion concentric texture by stirring the upstream pitch melt and using a capillary with large diameter.

\subsubsection{Structure Development}

Melt spinning is a complex non-isothermal process involving uniaxial flow, cooling and solidification. After leaving the spinneret, the melt pitch is cooled and solidified to form a fiber. This thermal relaxation involves heat transfer from the melt pitch to the cooling medium, which creates a radial temperature gradient across the fiber. Moreover, it is also during this thermal relaxation that the flow-induced structure starts to relax from a stressed high-energy to a low-energy state. The orientation of the molecules evolves from a random to an ordered structure during the thermal relaxation period. The reorientation process is sensitive to the melt viscosity and the cooling rate. High viscosity and fast quenching hinder the order development, causing a random texture. Lower viscosity and slow cooling impart the molecule reorientation and lead to an ordered structure [14].

\subsubsection{Structure and Properties Correlation}

It is the well-defined structures that give the mesophase pitch based carbon fibers excellent mechanical and transport properties. Studies [4] show that the high-degree of 
molecular orientation develop higher modulus, and the strengths can be increased by controlling the molecular orientation during fiber formation. It is also found that linearizing molecular orientation during fiber formation can yield carbon fibers with enhanced thermal conductivities $[15,16]$. Although considerable progress has been made over the past four decades, the origin and development of structure development and texture formation is still not completely understood for mesophase pitch based carbon fibers. Currently research work is being made to control the molecule orientation of fibers in order to optimize preferred properties, such as thermal conductivity.

\subsection{Thesis Objectives}

The overall goal of this thesis is to study the carbon fibers transverse texture formation and structure development for mesophase pitch based carbon fiber during the melt spinning process in a nonhomogenous temperature field. The aim is to investigate the effects of the process variables and material properties on the texture evolutions. The objectives of this thesis are as follows:

(1) To develop a mathematical model that describes the thermodynamic behaviour of liquid crystalline molecules under thermal relaxation process after steady extensional flow for a model incompressible uniaxial nematic pitch phase composed of rigid disc-like molecules using the Ericksen and Landau-de Gennes nematic continuum theories.

(2) To implement, solve and validate a one-dimensional model as specified and developed. To characterize the structure development and texture formation during thermal relaxation after cessation of extensional flow. An emphasis is placed on the understanding of the effect of process variables and material properties on the texture evolution.

(3) To implement, solve and validate a two-dimensional model as specified and developed. An emphasis is placed on the analysis of the structural morphology development from random to well-defined textures. To examine the effects of 
mesophase pitch material properties and process conditions on the texture formation and evolution.

(4) To explain the experimental observations reported in the literature on texture formed after cessation of extensional flow.

\subsection{Thesis Outline}

This thesis consists of six chapters. Chapters 1-3 provide necessary background knowledge. Chapters 4 and 5 show the one-dimension and two-dimension mathematical model development, and present the computer simulation results, respectively. The last chapter summarizes the conclusions drawn from the study and provides recommendations for future work. The contents of each chapter are described below:

Chapter 1 is a general introduction to this thesis study. It provides the motivation, objectives, and method of study and organization of the thesis.

Chapter 2 presents the theoretical background that is required for the mathematical model development in the study. It includes the liquid crystal basic concepts, the Frank elastic free energy theory, the Leslie-Ericksen theory, and Landau de-Gennes free energy theory.

Chapter 3 summarizes the literature review for the study, it provides reviews on the formation of mesophase pitch, and the process effects on the texture formation and properties of the mesophase pitch based carbon fibers. The exiting models that describe such process are also analyzed.

Chapter 4 presents the development of one-dimensional mathematical model and numerical method that is used for the thesis. The numerical solution techniques for solving these equations are also presented. Furthermore the chapter presents the numerical modeling and simulation results for texture formation and structure 
development at the thermal (i.e., cooling) relaxation process. The process parameters and mesophase pitch properties effects on texture evolution are also discussed.

Chapter 5 presents the two-dimensional numerical modeling development and results for structure development and texture formation. It also includes the governing auxiliary equations that are used for achieving the objectives of the model. The process parameters such as cooling rate and mesophase pitch properties (such as the melt texture, anchoring behaviour) effects on texture selection and evolutions are discussed.

Chapter 6 presents the conclusions for the presented work. Simulation results from onedimension and two-dimension model are compared. The contributions to current study and the recommendations for future studies are presented.

\subsection{Methodology}

In this thesis the following steps were followed in the computational modeling of structure formation of mesophase pitch based carbon fibers.

Step1: Specify the system under observation, the independent variables (time and space dimensions), and the dependent variable of the model. The dependent variables are the fibers temperature profile, the scalar order parameter, and the planar director orientation angle.

Step 2: Derive the governing time-dependent partial differential equations to describe the structure formation process using the Ericksen and Landau-de Gennes nematic continuum theories $[17,18]$.

Step 3: Determine the arbitrary initial and boundary conditions to solve the governing equations. The initial condition represent the melt texture formed before the thermal relaxation process, and the boundary conditions mimic the anchoring behaviours of nematic liquid crystalline molecules. 
Step 4: Nondimensionlize the governing equations, the initial and boundary conditions obtained in above steps.

Step 5: Develop the Galerkin finite element formulations of the dimensionless governing equations

Step 6: Implement and solve the Galerkin Finite Element formulations obtained in step 5 by writing a FORTRAN code.

Step 7: Analyze the computer simulation results obtained in step 6 and present them in scientific visualization methods using software AXUM and MATLAB.

Step 8: Interpret the simulation results mathematically and physically.

Step 9: Validate the mathematical model using published experimental observations. 


\section{Chapter 2}

\section{Technical Background}

This chapter provides the technical background for the mathematical modeling of mesophase pitch based carbon fibers structure development and texture formation. As mentioned before, mesophase pitch based carbon fibers are made from liquid crystalline materials, where the structure of the fiber originates from the mesophase pirch molecular orientations. Theoretical study of the texture formation in these carbon fibers requires the understanding of the physics of liquid crystal molecules. Therefore this chapter will briefly introduce the fundamentals of liquid crystal physics needed to model the mesophase pitch molecular behavior. This includes the definition of mesophases, director and the scalar order parameter, and the continuum theories of liquid crystals used in the mathematical model.

\subsection{Liquid Crystal Basic Concepts}

\subsubsection{Definition of Liquid Crystal Phases (Mesophases)}

Liquid crystal (mesophase) is a name for an intermediate phase between solid and liquid phases. Like mesophase pitch, many organic materials uridergo more than one intermediate phase before heated into liquid phase from solid state [17]. These phases, also called mesophases, have both liquid-like fluidity and crystalline molecular order. The phases flow like a liquid. However, their molecules maintain some degree of rotational order. The flow behavior of mesophase is much more complex than that of simple fluid flow due to the simultaneous effect of orientation and fluid flow. 


\subsubsection{Liquid Crystal Classification}

According to how the phase transition is effected, liquid crystal phases are classified as thermotropic or lyotropic [17]. Lyotropic mesophase is obtained by changing solute concentration or adding certain components. On the other hand, thermotropic phase transition is usually effected by temperature changes. Thermotropic materials can be processed by melt spinning or injection molding at temperature range when they are liquid crystal meli [18].

Liquid crystal phases are further classified into nematic, cholesteric and smectic according to the phase symmetry. The three different phases are shown in Figure 2.1. In a nematic phase the molecules tend to align parallel to each other. The preferred average molecular orientation is defined as director $\mathbf{n}$, which is a unit vector [19]. Cholesteric phase has a similar nematic order on a local scale, i.e., the molecules tend to align with the director. However, on the larger scale, the director exhibits a helical pattern. Smectic phase has a layered structure and the molecules are aligned paraliel within the layer. There are several types of smectic phases. Figure 2.1c depicts the smectic-A phase.

Compared to smectic and cholesteric phases, the nematic phase has the following characteristics: (1) nematic has cylindrical symmetry; and (2) nematic phase has longrange orientational order but no long-range translational order. Although mesophase pitch behaves as either thermotropic or lyotripic, it is usually modeled as a discotic nematic thermotropic phase [9]. 


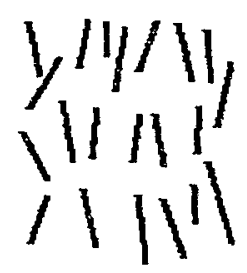

(a)

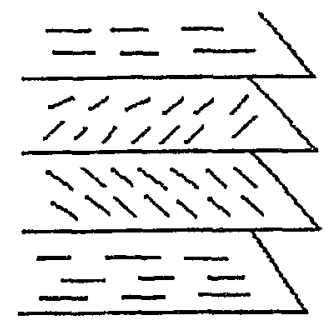

(b)

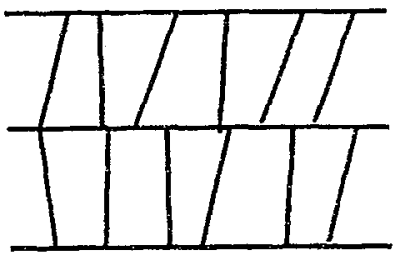

(c)

Figure 2.1 The liquid crystal phases: (a) Nematic, (b) Cholesteric and (c) Smectic-A.

\subsubsection{Orientational Order}

In a nematic phase, the molecules tend to align parallel to each other. The orientational order has uniaxial symmetry. This preferred direction is defined by the director $\mathbf{n}$. This unit vector represents the local mean direction of molecules. As Figure 2.2 shows $\mathbf{n}$ is parallel to the long axis in a rod-like nematics, and normal to the molecules if the molecule is discotic. A second local variable is the scalar order parameter, $S$, which is a measurement of the degree of molecular alignment along the director.

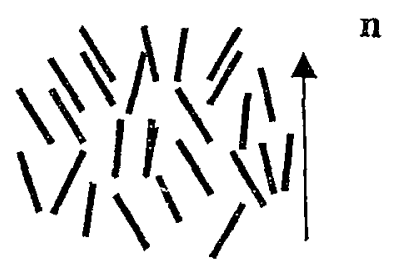

(a)

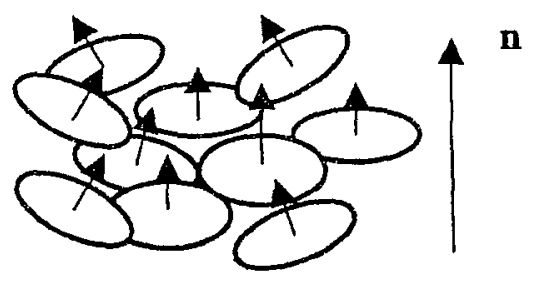

(b)

Figure 2.2 Schematic representation of molecular director in (a) rod-like nematic and (b) discotic nematic phases 
The order parameter for a nematic liquid crystal is expressed as [17-22]:

$S=\frac{1}{2}\left(3<\cos ^{2} \theta>-1\right)$

where $\theta$ is the angle between the molecule symmetry axis and the director $\mathbf{n}$, as shown in Figure 2.3. For rod-like molecules, $\theta$ is the angle between molecules and director $\mathbf{n}$; whereas, for discotic molecules, $\theta$ is the angle between the disc normal and the director n. $\left\langle\cos ^{2} \theta>\right.$ represents averages of all the molecules in the nematic phase. When all the molecules are randomly distributed in any direction, the value of the average is $1 / 3$, which results in $S=0$ for the disordered isotropic phase. When all the molecules are fully aligned with $\mathbf{n}$, all $\theta=0$ which results in $\left\langle\cos ^{2} \theta\right\rangle=1$, and $S=1$ for the completely ordered nematic phase.

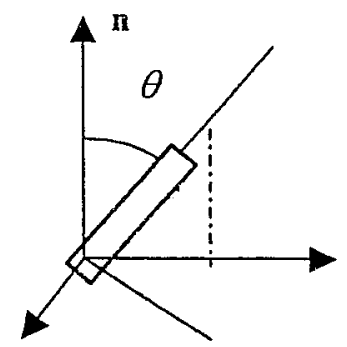

(a)

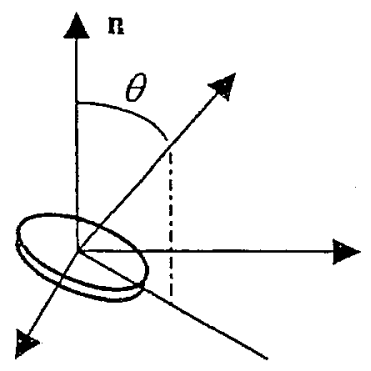

(b)

Figure 2.3 Schematic representation of orientation angle $\theta$ in

(a) rod-like nematic and (b) discotic nematic 
A value between zero and unity describes degrees of ordering intermediate between completely isotropic and totally ordered. The typical value of the scalar order parameter for nematic is $0.4<S<0.8$ [17]. Figure 2.4 shows schematically how the scalar order parameter varies for the temperature-effected thermotropic and concentration-effected lyotropic. $T_{\mathrm{Nl}}$ is defined as critical temperature where the first-order transition occurs for thermotropic liquid crystal; $c_{\mathrm{NI}}$ is defined as critical concentration where the first-order transition occurs for lyotropic liquid crystal [17].

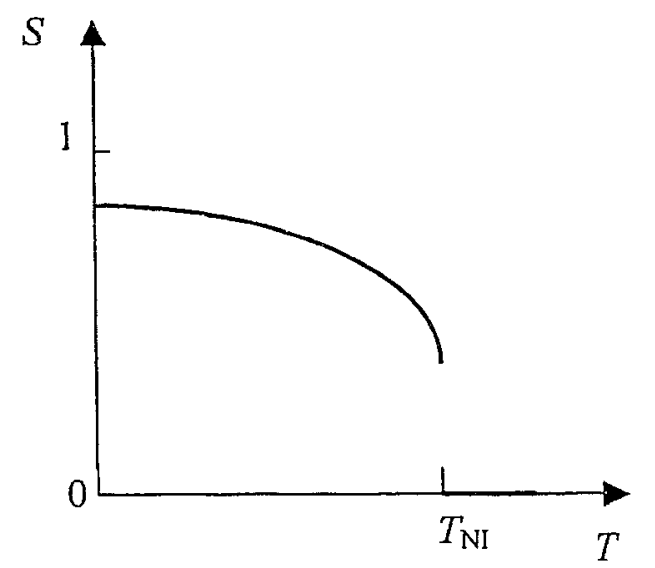

(a)

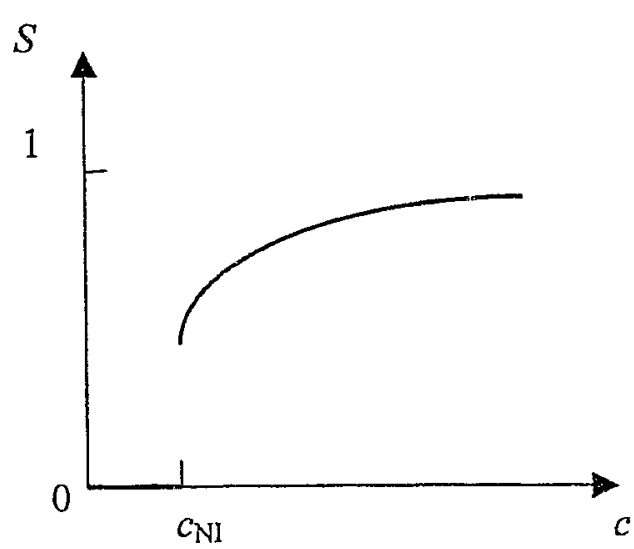

(b)

Figure 2.4 Typical variations of the scalar order parameter $S$ for (a) thermotropic liquid crystals with temperature $T$ and $(b)$ lyotropic liquid crystals with concentration $c$. And $T_{\mathrm{NI}}$ and $c_{\mathrm{NI}}$ are the first order transition points. 


\subsection{Frank-Oseen-Zocher Static Elastic Continuum Theory}

When we consider a spatial region inside a macroscopic sample of nematic liquid crystal, it contains a sufficiently large number of molecules so that the long-range orientational order is well defined within that region. In each of the region we have an orientational director $\mathbf{n}$. The variations of $\mathbf{n}$ are only important over distance much larger than the molecular dimensions. A nematic can still be taken locally uniaxial, and $S$ can then be considered the average degree of orientation with respect to the local director. In other words, the spatial dependence of $\mathbf{n}$ and the temperature dependence of $S$ can be treated separately. For a definite value of $S(T)$, the nematic sample may consists different arrangement of director $\mathbf{n}(\mathbf{r})$. This is the basis of the following continuum theory of liquid crystals [19].

In an ideal nematic single liquid crystal, the molecules are aligned along one common director $\mathbf{n}$. If the system is imposed by external fields, such as magnetic, electric or the limiting surface, the orientation of director $\mathbf{n}$ will change from point to point. The distorted state is described in terms of a vector field $\mathbf{n}(\mathbf{r})$. $\mathbf{r}$ is defined as the position of the single liquid crystal in the uniaxial liquid crystal system. The unit length director $\mathbf{n}(\mathbf{r})$ varies its orientation slowly and smoothly everywhere.

The spatial gradients of $\mathbf{n}$ increase the elastic free energy density of the system. If the gradient of the director is small enough to ignore the change in $\mathbf{n}$ over the length of a single molecule, the liquid crystals can be described as a continuous medium with a set of elastic constants. Then the increased free energy $F_{\mathrm{d}}$ invoked by the distortion of $\mathbf{n}$ can be described by a continuum theory. Oseen [23], Zocher [24] and Frank [25] derived the following formula of the elastic free energy density using the cylindrical symmetry of nematic phases: 
$f_{\mathrm{d}}=\frac{1}{2} K_{1}(\nabla \cdot \mathbf{n})^{2}+\frac{1}{2} K_{2}(\mathbf{n} \times \nabla \cdot \mathbf{n})^{2}+\frac{1}{2} K_{3}(\mathbf{n} \times \nabla \times \mathbf{n})^{2}$

where $K_{1}, K_{2}$, and $K_{3}$ are the splay, twist and bend temperature-dependent elastic constants, respectively, and are named collectively as the Frank elastic constants. The type of liquid crystal elasticity is known as orientation elasticity. Figure 2.5 shows the three types of elastic deformation, splay, twist, and bend, and their respective modulus $K_{1}, K_{2}$, and $K_{3}[19]$.

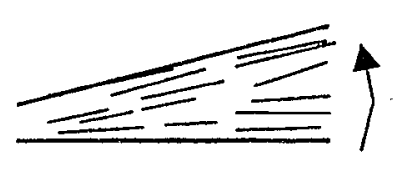

$K_{1}$

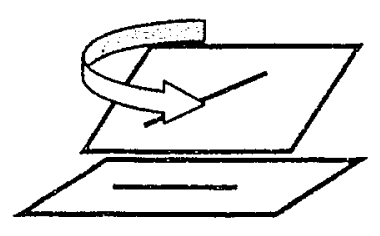

$K_{2}$

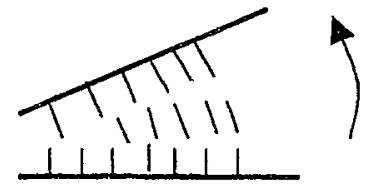

$K_{3}$

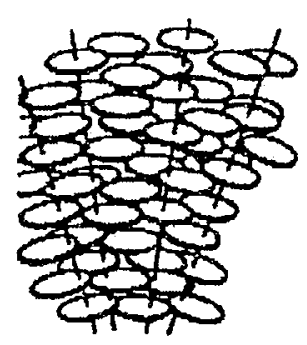

$K_{1}$

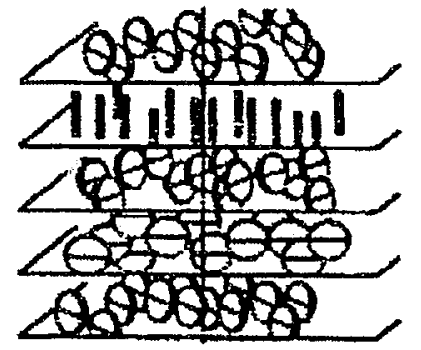

$K_{2}$

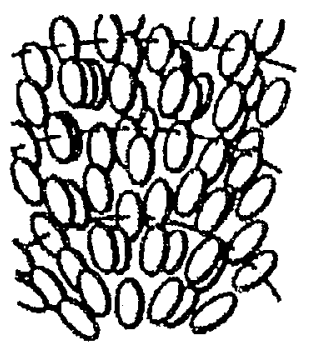

$K_{3}$ (b)

Figure. 2.5 Three types of deformation in nematics: (a) rod-like molecules and (b) discotic molecules. $K_{1}$-splay deformation, $K_{2}$-twist deformation and $K_{3}$-bend deformation 
The common values of the set of elastic constants are on the order of $10^{-12} \mathrm{~N}$ [19]. For rod-like nematic liquid crystals, $K_{3}$ is larger than $K_{1}$ and $K_{2}$ [19], while for disc-like nematic liquid crystals, the twist constant is larger than $K_{1}$ and $K_{3}$ [20]. Usually, the oneconstant approximation is assumed which means that the nematic is elastically isotropic; i.e., $K=K_{1}=K_{2}=K_{3}$ and $f_{\mathrm{d}}$ simplified as [19]:

$$
f_{\mathrm{d}}=\frac{1}{2} K\left[(\nabla \cdot \mathbf{n})^{2}+(\nabla \times \mathbf{n})^{2}\right]
$$

The Frank elastic free energy theory is considered the fundamental of the continuum theory for the cylindrical symmetry of nematic liquid crystals. It means the elastic free energy density $f_{\mathrm{d}}$ comes from three kinds of direction deformations: splay, twist and bend. In another words, the strains of a liquid crystal material are due to the spatial orientation gradients, and are analogous to positional displacements in isotropic materials. The Frank elastic constants play the role of Hocke's modulus of isotropic materials.

\subsection{Leslie-Ericksen Continuum Theory}

The Leslie-Ericksen continuum theory was developed initially for rod-like nematic liquid crystals. It considers the orientation of molecule rotation, and thus requires the incorporation of the Frank free energy density. Due to the coupling between molecular orientation and fluid flow, the flow behaviour of the nematic liquid crystals is much more complex than that of the conventional fluid flow. The following simplified interpretation of the Leslie-Ericksen continuum theory considers the following assumptions:

(a) The nematic is incompressible,

(b) The nematic order parameter $S$ is spatially homogenous, and

(c) Inertial terms in the equation are negligible. 


\subsubsection{Mass Balance}

By applying the conservation law of mass, the mass balance in Cartesian tensorial notation for an incompressible fluid balance equation is $[9,19]$ :

$(\nabla \cdot V)=0$

where $\mathbf{V}$ is the velocity of the nematic phase. The equation is same as an incompressible fluid flow.

\subsubsection{Linear Momentum Balance}

In Cartesian tensorial notation for an incompressible fluid, the linear momentum balance equation is [20]:

$\rho \dot{\mathbf{V}}=\mathbf{F}+\nabla \cdot \boldsymbol{\tau}$

where $\rho$ is the fluid density, $\mathbf{V}$ is the velocity, and $\mathbf{F}$ is the external body force per unit volume. The superposed dot denotes the material time derivative. $\tau$ is the viscous stress tensor and its constitutive expression is given as [21]:

$$
\begin{aligned}
\boldsymbol{\tau}= & -p \boldsymbol{\delta}-\frac{\partial f_{\mathrm{d}}}{\partial \nabla \mathbf{n}} \cdot(\nabla \mathbf{n})^{\mathbf{T}}+\alpha_{1}(\mathbf{n n}: \mathbf{A}) \mathbf{n} \mathbf{n}+\alpha_{2} \mathbf{n N}+\alpha_{3} \mathbf{N} \mathbf{n}+\alpha_{4} \mathbf{A}+ \\
& \alpha_{5} \mathbf{n} \mathbf{n} \cdot \mathbf{A}+\alpha_{6} \mathbf{A} \cdot \mathbf{n n}
\end{aligned}
$$

where the kinematic quantities are defined as [21]:

$$
\begin{aligned}
& \mathbf{A}=\frac{1}{2}\left[(\nabla \mathbf{V})^{\mathrm{T}}+\nabla \mathbf{V}\right] \\
& \mathbf{N}=\dot{\mathbf{n}}-\mathbf{\Omega} \cdot \mathbf{n}
\end{aligned}
$$


$\Omega=\frac{1}{2}\left[(\nabla \mathbf{V})^{\mathrm{T}}-\nabla \mathbf{V}\right]$

In equation (2.7a-c), the $\left\{\alpha_{i}\right\}, i=1, \ldots, 6$ are known as the viscosity coefficients, $p$ is the pressure and $\boldsymbol{\delta}$ is the unit tensor. $\mathbf{A}$ is the rate of deformation tensor, $\mathbf{N}$ is the angular velocity of the director relative to that of the fluid, and $\mathbf{\Omega}$ is the vorticity tensor. Compared with ordinary isotropic flow, nematic flow has six viscous terms in the stress tensor, because the theory accounts the fact that the stress tensor depends not only on the velocity gradients, but also on the orientation and rotation of the director. Note that all the terms on the right hand side involve the director orientation except the fourth term $\alpha_{4} \mathbf{A}$, which is the same term that for an isotropic fluid $\alpha_{4}=2 \eta$ [9].

\subsubsection{Angular Momentum Balance}

The tensorial notation of the internal angular momentum balance equations in Cartesian coordinate for an incompressible fluid is:

$[\mathbf{n} \times \mathbf{h}]=-\left(\alpha_{3}-\alpha_{2}\right)[\mathbf{n} \times \mathbf{N}]-\left(\alpha_{3}+\alpha_{2}\right)[\mathbf{n} \times[\mathbf{A} \cdot \mathbf{n}]]$

where $\mathbf{h}$ is the molecular field. The angular momentum balance governs the director angular motion. The right hand side of the equation (2.8) defines the elastic torque $\Gamma_{\mathrm{e}}$ on the director per unit volume. The molecular internal elastic field arises from the elasticity of the nematic material [22]. Differentiation of the equation (2.2) yields the expression for the molecular internal elastic field defined as follows [9, 19-21]:

$$
\begin{aligned}
& \mathbf{h}=\mathbf{h}_{\mathbf{S}}+\mathbf{h}_{\mathbf{T}}+\mathbf{h}_{\mathbf{B}} \\
& \mathbf{h}_{\mathbf{S}}=K_{1} \nabla(\nabla \cdot \mathbf{n}) \\
& \mathbf{h}_{\mathbf{T}}=-K_{2}[(\mathbf{a} \nabla \times \mathbf{n})+\nabla \times(\mathbf{a n})]
\end{aligned}
$$




$$
\begin{aligned}
& \mathbf{h}_{\mathbf{B}}=K_{3}[(\mathbf{b} \times \nabla \times \mathbf{n})+(\nabla \times(\mathbf{n} \times \mathbf{b})] \\
& \mathbf{a}=\mathbf{n} \cdot \nabla \times \mathbf{n} \\
& \mathbf{b}=\mathbf{n} \times \nabla \times \mathbf{n}
\end{aligned}
$$

The left hand side of equation (2.8) expresses the viscous torque on the director. Viscous torque consists of two components: the first component is pure rotational effect expressed as [22]:

$$
\gamma_{1} \mathbf{n} \times \mathbf{N}
$$

The other component comes from the coupling to the fluid motion expressed as [22]:

$\gamma_{2} \mathbf{n} \times[\mathbf{A} \cdot \mathbf{N}]$

$\gamma_{1}$ is the rotational viscosity and $\gamma_{2}$ is the irrotational viscosity related to the Leslie coefficients by

$\gamma_{1}=\alpha_{3}-\alpha_{2}$

$\gamma_{2}=\alpha_{6}-\alpha_{5}=\alpha_{3}+\alpha_{2}$

Parodi [20] derived the equality in equation; thus there are only five independent Leslie viscosities.

Therefore equation (2.8) is simplified as:

$\Gamma_{\mathbf{e}}+\Gamma_{\mathbf{v}}=0$ 


\subsection{Landau-de Gennes Nematic Continuum Theory}

Frank elastic free energy density describes the energy increased in a nematic phase when liquid crystal molecules deviate from a uniform phase. At equilibrium, the Helmholtz free energy density of a nematic system can be approximated by the following low order polynomial expression in $S$ [19]:

$f_{\mathrm{S}}=f_{\mathrm{o}}(T)+\frac{3}{4} A\left(T-T_{\mathrm{NI}}{ }^{+}\right) S^{2}+\frac{1}{4} B S^{3}+\frac{9}{16} C S^{4}$

where $f_{0}(T)$ is the isotropic free energy density at temperature $T$, and $A, B, C$, are material constants. $T_{\mathrm{NI}}{ }^{+}$is a temperature slightly below the clearing temperature where the first order transition occurs. It is also expressed as $T_{\mathrm{C}}{ }^{*}$ in liquid crystal literatures $[17,19]$.

When the scalar order parameter $S$ is constant across the sample, the nematic is characterized by the director field $\mathbf{n}(\mathbf{r})$. If there is variation of temperature across the sample, the scalar order parameter $S$ will be defined as $S(\mathbf{r})$. The nematic free energy density induced by director gradient is not simply given by Frank expression, but also contains terms coming from the gradients of the scalar order parameter $S$ [17].

Landau [17] made a far-reaching speculation about the functional dependence of the freeenergy density on the order parameter and its spatial derivatives near a second-order transition point. The theory is intended to express the second-order phase transition, but it can be generalized to include the first-order phase transitions [17]. The free energy density is expressed as a power series in terms of one or more long-range order parameters and their spatial derivatives. Since only the leading terms are important near the transition point, the resulting expansion is a low-order polynomial with temperature dependent coefficients. de Gennes [19] later applied Landau's theory to the first order phase transition of nematic phases successfully. For a nematic phase, the order parameter is the scalar order parameter defined by equation (2.1). 
The Landau-de Gennes free energy density of the nematic material is defined as follows [17]:

$$
\begin{aligned}
f_{\mathrm{L}} & =f_{\mathrm{o}}(T)+\frac{3}{4} A\left(T-T_{\mathrm{NI}}{ }^{+}\right) S^{2}+\frac{1}{4} B S^{3}+\frac{9}{16} C S^{4} \\
& +\frac{3}{4}\left(L_{1}+\frac{1}{6} L_{2}\right)(\nabla S)^{2}+\frac{3}{8} L_{2}(\mathbf{n} \cdot \nabla S)^{2} \\
& +\frac{9}{4} S^{2}\left[\left(L_{1}+\frac{1}{2} L_{2}\right)(\nabla \cdot \mathbf{n})^{2}+L_{1}(\mathbf{n} \cdot \nabla \times \mathbf{n})^{2}+\left(L_{1}+\frac{1}{2} L_{2}\right)(\mathbf{n} \times \nabla \times \mathbf{n})^{2}\right] \\
& +\frac{3}{2} L_{2} S(\nabla \cdot \mathbf{n}) \times(\mathbf{n} \cdot \nabla S)+\frac{3}{4} L_{2} S(\mathbf{n} \times \nabla \times \mathbf{n}) \cdot \nabla S
\end{aligned}
$$

Equation (2.15) contains four groups of terms. The first four terms contain only the scalar order parameter. The next two terms account for the spatial variations in $S$. The following three terms account for director spatial variation, and is expanded as such to resemble the Frank-Oseen-Zocher free energy density, which is expressed as equation (2.2). The last two terms in equation (2.15) represent the interaction between spatial variations of $S$ and spatial variations of $\mathbf{n}$. In this paper these four group of terms in equation (2.15) are conveniently called the molecular free energy density $f_{S}$, the molecular elastic free energy density $f_{\mathfrak{e}}$, the Frank elastic free energy density $f_{\mathrm{d}}$, and the coupling elastic free energy density $f c$, respectively [27].

It should be noted that to second order in the Landau expression there are only two independent elastic constants, $L_{1}$ and $L_{2}$ : whereas in the Frank-Oseen-Zocher free energy density there are three independent elastic constants $K_{1}, K_{2}$ and $K_{3}$. They may be related as follows with the scalar order parameter $S[26]$ :

$L_{1}=\frac{K_{2}}{2 S^{2}}$ 
$L_{2}=\frac{K-K_{2}}{2 S^{2}}$

$K=K_{1}=K_{3}$

\subsection{Ericksen Continuum Theory for Nonhomogenously Ordered Nematic Phases}

The Leslie-Ericksen continuum theory describes the dynamic behavior of the nematic directors in an homogenously ordered system and incorporates the Frank elastic free energy density. Ericksen [28] later proposed a modified version of the Leslie-Ericksen continuum theory to model the more complex behavior of nematic directors in a nonhomogenously ordered phase. The modified version contains the essential terms in the elementary static models used to describe the nematic and its scalar order parameter gradient. The most significant changes to the Leslie-Ericksen theory are the addition of $S$, and its spatial and temporal gradients.

The balance equations for $\mathbf{n}$ and $S$ are defined, respectively, as follows [28]:

$$
\begin{aligned}
& \dot{\mathbf{n}}=\mathbf{\Omega} \cdot \mathbf{n}+\frac{\gamma_{2}^{S}(S)}{\gamma_{1}^{S}(S)}\left[\mathbf{n}\left(\mathbf{n}^{\mathbf{T}} \cdot \mathbf{A} \cdot \mathbf{n}\right)-\mathbf{A} \cdot \mathbf{n}\right]-\frac{1}{\gamma_{1}^{S}(S)} \frac{\delta f_{\mathrm{L}}}{\delta \mathbf{n}} \\
& \dot{S}=-\frac{1}{\beta_{2}^{S}(S)} \frac{\delta f_{\mathrm{L}}}{\delta S}-\frac{\beta_{1}^{S}(S)}{\beta_{2}^{S}(S)} \mathbf{n}^{\mathrm{T}} \cdot \mathbf{A} \cdot \mathbf{n}
\end{aligned}
$$

The superposed dot denotes the material time derivative, and $\delta f_{\mathrm{L}} / \delta(*)$ denotes the functional derivative of $f_{\mathrm{L}}$ with respect to $\left(^{*}\right)$. The superscript $S$ denotes that the material properties change with order parameter $S$. The rotational and irrotational viscosities given in equation (2.10) and (2.11) as $\gamma_{1}$ and $\gamma_{2}$ now becomes $\gamma_{1}^{S}$ and $\gamma_{2}^{S}$. The two new 
viscosities introduced by the balance equation for $S$, i.e., $\beta_{1}^{S}$ and $\beta_{2}^{S}$, are also dependent on $S$. Other terms are already defined in Section 2.3. The constitutive equation for the stress tensor is defined as:

$$
\begin{aligned}
\boldsymbol{\tau}= & -p \boldsymbol{\delta}-\frac{\partial f_{\mathrm{L}}}{\partial \nabla \mathbf{n}} \cdot(\nabla \mathbf{n})^{\mathrm{T}}-\frac{\partial f_{\mathrm{L}}}{\partial \nabla S} \nabla S+\beta_{1}^{S}(S) \dot{S} \mathbf{n} \mathbf{n}+\alpha_{1}^{S}(S)(\mathbf{n n}: \mathbf{A}) \mathbf{n n} \\
& +\alpha_{2}^{S}(S) \mathbf{n} \mathbf{N}+\alpha_{3}^{S}(S) \mathbf{N n}+\alpha_{4}^{S}(S) \mathbf{A}+\alpha_{5}^{S}(S) \mathbf{n n} \cdot \mathbf{A}+\alpha_{6}^{S}(S) \mathbf{A} \cdot \mathbf{n} \mathbf{n}
\end{aligned}
$$

where now the Leslie viscosities $\left\{\alpha_{\mathrm{i}}\right\}, i=1, \ldots, 6$ become $\left\{\alpha_{\mathrm{i}}^{\mathrm{s}}\right\}, i=1, \ldots, 6$ and are also dependent on $S$. Ericksen derived expressions in terms of $S$ for these viscosities, but they all contain undetermined coefficients. Beris and Edwards [26] later simplified these formulation and obtained new expressions for these viscosities. The relationships are listed in the Appendix.

\subsection{Nematic Potential and Equilibrium Orientational Order}

Doi and Edwards [29] give a special form for the molecular free energy density of the Landau-de Gennes equation using the mean field theory. They assumed all molecules are rigid and undergo Brownian motion and obtained:

$$
f_{\mathrm{S}}=k_{\mathrm{B}} \cup T\left[\frac{1}{2}\left(1-\frac{1}{3} U\right) S^{2}-\frac{1}{9} U S^{3}+\frac{1}{6} U S^{4}\right]
$$

where $k_{\mathrm{B}}$ is the Boltzmann constant, $v$ is the disc concentration, and $T$ is the absolute temperature. The nematic potential $U$ is the dimensionless temperature for thermotropic or dimentionless concentration for lyotropic system, expressed as [29]:

$$
U=\frac{3 T_{\mathrm{Nl}}}{T}
$$

or 


$$
U=\frac{3 c_{\mathrm{NI}}}{c}
$$

Doi and Edwards [29] further analyzed equation (2.19) to predict the phase transition under static condition and gave a general range of nematic potential for different phases, which is depicted in Figure 2.6.

For $U<8 / 3$, the free energy only has one minimum at $S=0$. The stable phase for this range of potential is isotropic.

For $8 / 3<U<3$, the free energy profile has two local minima, one is at $S=0$, and the other at:

$S=\frac{1}{4}+\frac{3}{4}\left(1-\frac{8}{3 U}\right)^{1 / 2}$

For this range of potential, there exists equilibrium between nematic and isotropic liquid phases.

For $U>3$, there is only one minimum. The point $S=0$ is not a minimum, and the phase becomes unstable; the system always approaches the nematic state. 


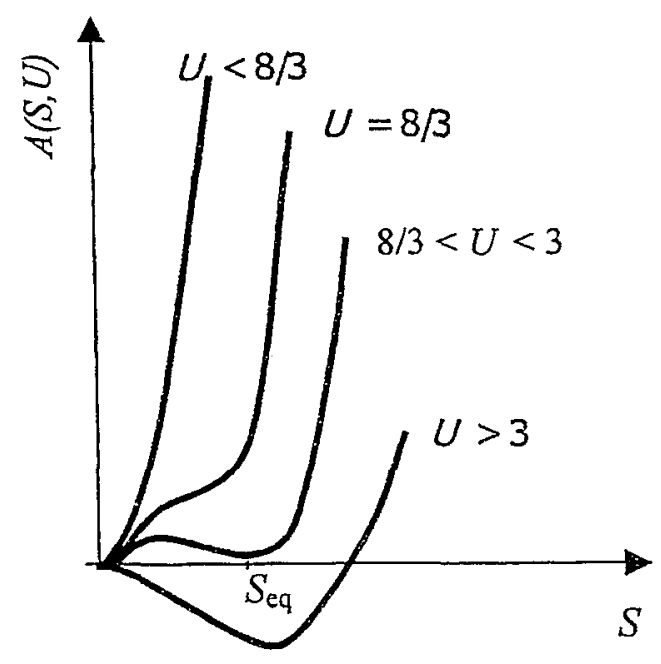

Figure 2.6 Typical dependence of the free energy on the scalar order parameter $S$ [29]. $U<8 / 3$, the stable phase is isotropic, $8 / 3<U<3$, nematic and isotropic liquid phase coexist, $U>3$, the system always approaches the nematic state . 


\section{Chapter 3}

\section{Literature Review}

In this chapter, mesophase pitch liquid crystal properties, melt spinning process and process variables that affect mesophase pitch based carbon fiber structure and properties are introduced. The commonly observed textures from published production and experimental observations are also presented. Lastly, the simulation results from previous mathematical models are analyzed.

\subsection{Introduction to Mesophase Pitch}

Brooks and Taylor [30] first found that in a series of heat-treated coking tar pitch there is another anisotropic phase coexisting with isotropic phase under optical microscope with polarized light. Brooks and Taylor later defined the anisotropic intermediate phase as mesophase. The mesophase is formed by anisotropic spheres, which are aromatic molecules stacked together through oligomer formation, coalescence and adhesion. The optical anisotropy observed is due to the layered stacking of its aromatic sheet as shown in Figure 3.1 [2].

Mochida et al [31] later systemically analyzed the mesophase formation mechanism, its liquid crystalline properties, and its ordered structure due to molecular orientation utilizing optical microscopes, scanning electron microscopes and transmission electron microscopes. The planar molecules in the mesophase pitch stack together and form clusters due to Van Der Waals force. The clusters gather together to form microdomains, 
where the clusters are aligned basically in the same direction, as expressed in Figure 3.1 [2], the microdmains form the textural units of domains that define the macroscopic texture.

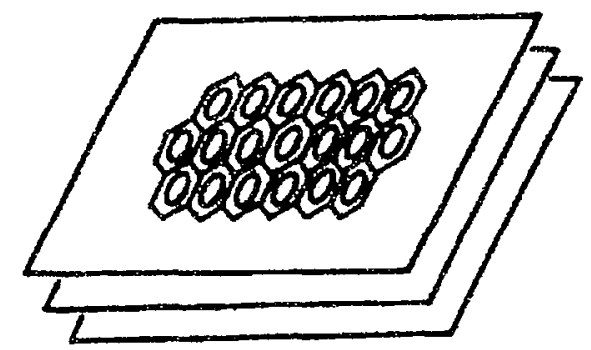

Coal tar derived mesophase

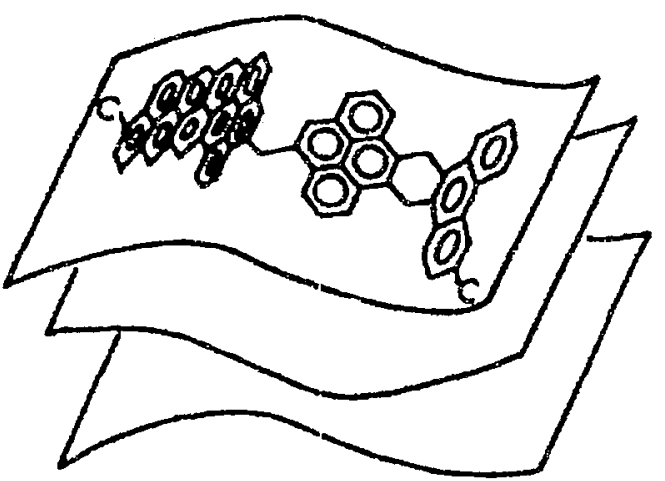

Petroleum derived mesophase

Fig. 3.1 Typical poly-nuclear aromatic hydrocarbons in mesophase produced from coal and petroleum [2].

Mesophase pitch has been found exhibiting both thermotropic and lytropic liquid crystal nature $[32,33]$. It remains anisotropic within a certain temperature range below the transition temperature. The mesophase existence also depends on the pitch composition. It disappears or reappears by adding or removing certain lower molecular weight components. Mesophase pitch also exhibits discotic liquid crystal structural properties. Despite the variety of formation method, the general molecules are disc shape, and the average molecular weight is $400-4000$ [31]. The melt flows in a liquid state above its soften point and maintains the molecule orientation order in a certain temperature range as it flows. The viscosity of the mesophase is very dependent on the temperature, shear rate, 
and mesophase structure. Unlike small molecular liquid, mesophase pitch flow has non-Newtonian behaviour. Nazem [34] compared the viscosity of mesophase pitch and isotropic pitch and other melt spun polymers and found mesophase pitch viscosity is far more temperature dependent than typical melt-spun polymer such as nylon-6. This comparison is shown in Figure 3.2.

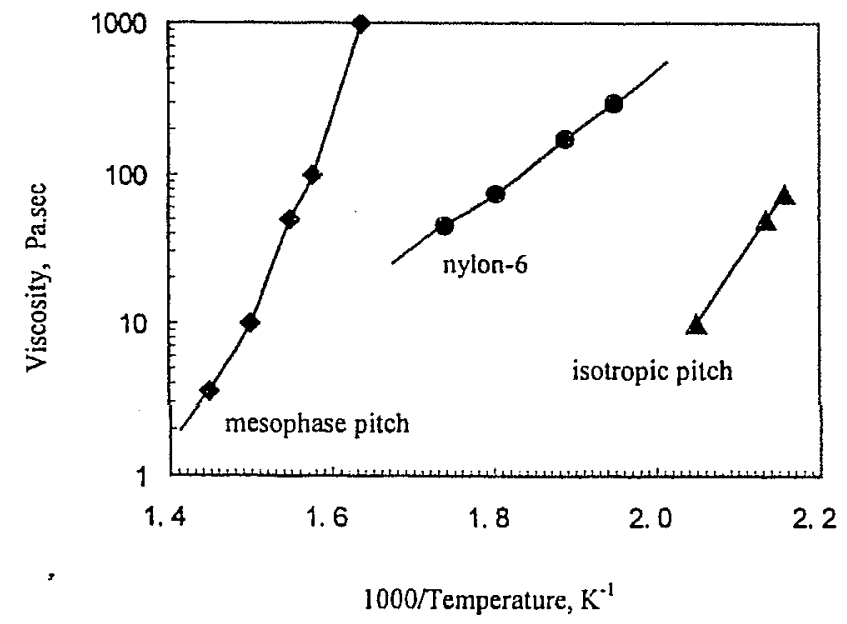

Figure 3.2 Dependency of viscosity on temperature for mesophase pitch, isotropic pitch and nylon-6 [34].

\subsection{Melt Spinning and Process Effect on Fibers Structures and Properties}

The methods of mesophase pitch preparation have been investigated since mesophase melt from petroleum or coal tar came into production in the 1950s. More recently, Singer [35] 
investigated the mesophase pitch spinnability and rlieology, and suggested that it might deform at high temperature by shear or elongational forces before it become an infusible coke. With his coworkers they first designed the monofilament spinning apparatus with piston feed. The first mesophase pitch based carbon fibers with highly oriented and organized structure were produced by Union Carbide in the 1960s. After stabilization, carbonization and graphitization the final fibers can achieve extremely high modulus approaching the theoretical limit of graphite $\left(10^{12} \mathrm{~Pa}\right)$ [35]. Ever since the very beginning of the mesophase pitch production, the correlation among the oriented structure, the properties and the spinning process had become the most interesting subject for experimental observation and theoretical studies.

\subsubsection{Melt Spinning Process and Process Parameters}

A typical melt spinning process is shown in Figure 3.3 [2]. After some preparational heat treatment mesophase pitch is loaded into a set of screw extruder where it is heated and mixed to form a viscous melt. Then the viscous melt is forced into the spin pack. Upon existing the spin pack, the extruded filaments are simultaneously quenched and drawn down by a winding device to form solid fibers. Spinning conditions for melt spinning mesophase pitch include: pitch properties (glass transition temperature $T_{\mathrm{g}}$, density $\rho$, viscosity $\gamma$ ), quench air temperature $T_{\mathrm{C}}$, spinning melt temperature $T_{\mathrm{S}}$, winder speed $V_{\mathrm{l}}$, cross-flow air velocity, spinneret diameter [36].

By applying energy, mass and force balances, Edie and Dunham [36] showed that the mesophase melt-spinning process is extremely sensitive to small changes in process conditions. Although the model did not use liquid crystal theories, their results still indicate that the spinning temperature and heat-treatment rate are the most important process variables among the melt spinning process parameters. This is because the 
mesophase pitch viscosity is extremely temperature sensitive compared to usual fluids. The model predicated a small change in process variables would dramatically affect fiber properties. However, the model could not predict the process effect on the structure formation since the model does not incorporate the physics of mesophase pitch molecules.

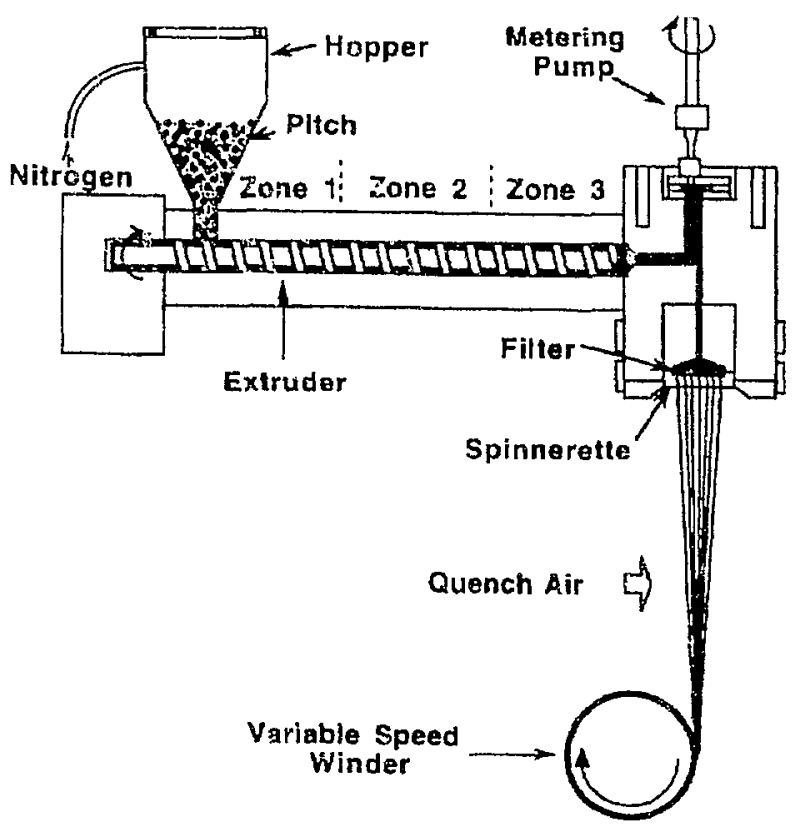

Figure 3.3 Melt spinning process [2].

\subsubsection{Process Variable Dependent Textures}

Hamada et al [13] and McHugh and Edie [37] concluded that different orientations of discotic molecules give rise to various ordered cross sectional textures. Previous work by Hamada et al [13], White and Buechler [14], McHugh and Edie [37] indicated that this 
ordered structure is created during melt spinning process; spinning parameters such as the melt spinning temperatzre, the heat treatment rate, and spinneret geometry all affect the structure texture. Hong et al [38] proved the mesophase pitch based carbon fibers texture is formed during melt spinning stage; the following post treatments only refine the formed texture. They compared the samples taken before and after heat treatment process. The transverse cross-sectional surface was observed by a high-resolution scanning electron microscope (HR-SEM). The as-spun samples were observed after platinum coating, and the heat-treated fibers at $700^{\circ} \mathrm{C}$ or higher without coating. The as-spun fibers spun at $300^{\circ} \mathrm{C}, 310^{\circ} \mathrm{C}$, and $340^{\circ} \mathrm{C}$ exhibit radial, random, and onion textures, respectively. The heat-treated fiber spun at $300^{\circ} \mathrm{C}, 310^{\circ} \mathrm{C}$, and $340^{\circ} \mathrm{C}$ exhibit radial texture with open crack, random, and onion with voids in the fiber center, respectively. They concluded high temperature induces onion structure, low temperature would yield radial structure, and intermediate temperature favors a random structure. White and Buechler [14], however, argued that the random texture results from high viscosity and rapid quenching, while the radial texture would form at lower viscosity and slow cooling.

\subsubsection{Different Textures Observed from The Experimental Work}

In order to explore carbon fibers application, some experimental work is performed to obtain different transverse textures. Hamada et al [13] changed transverse structure by disrupting the flow profile prior to extrusion. Another important experimental observation is the effect of spinneret geometry on the transverse texture formation. Matsumoto [12] and Edie et al [16] used extrusion capillaries with noncircular cross-sections to produce ribbon shaped carbon. The ribbon shaped fiber has high degree of orientation than the commonly produced round-shaped fiber. The experimental results show this ribbon shaped fiber has superior thermal and electrical conductivity and require lower graphitization temperature than the round-shaped fiber. Fortin et al [39] also reported that 
their thin fiber tape extruded from slit-shaped nozzle exhibits $2 \%$ higher degree of orientation than the round shaped fiber spun through circular nozzle under the some spinning conditions. Edie [2] further predicated that this could extend carbon fiber to a new field application where thermal transfer is critical. Gallego and Edie [15] suggested if the degree of the order of the structure could be improved during melt spinning process, it will greatly reduce the production cost by decreasing the graphitization temperature.

\subsubsection{Texture Dependent Properties}

Robinson and Edie [40] indicated that different observed textures exhibit different mechanical and transport properties. For example, mesophase fibers with random textures have higher tensile and compressive strength. By contrast, fibers with ordered transverse textures appear to develop better lattice dependent properties, such as thermal conductivity and electrical conductivity. Bright and Singer [41] also found that the transverse texture could affect the degree of graphitization. Fibers with radial texture developed large crystalline with higher degree of orientation than that of fibers with random texture. The properties, the texture formation and the mode of growth have been the subject of numerous articles [12-16,39].

Beginning with the work by Matsumoto [12], Hamada et al [13], and McHugh and Edie [37], it became apparent that the texture of mesophase fibers was created and thus could be controlled during melt spinning. Since that time numerous researchers have demonstrated that flow during melt spinning can be controlled to create mesophase fibers with a variety of textures, each with certain advantages. 


\subsection{Mathematical Models}

Theoretical studies using available liquid crystal continuum theories to model and simulate the ordered structure selection process have been performed by McHugh and Edie $[9,37]$ and Singh and Rey [42-46]. McHugh and Edie [9,37] modeled mesophase pitch molecules as rigid discotic nematics and studied their molecular orientation during fully developed channel flow. Their numerical results were consistent with pitch observed under a polarized light microscope. Singh and Rey [42-46] examined continuum theories such as Leslie-Ericksen theory developed for rod-like liquid crystals and proposed that the theories can be extended to low molecular weight disc-like liquid crystal molecules to describe the flow behaviour of mesophase pitch. Several studies have analyzed the effect of spinning variables on the structure formation of mesophase pitch based carbon fibers. Spinning temperature and die geometry appear to be the most important variables [47-50].

\subsubsection{Wang and Rey's Elastic Model}

Wang and Rey [50] approximated the melt pitch to be a nematic discotic liquid crystal and assumed the melt spinning process to be an isothermal, incompressible, uniaxial, extensional flow. They modeled the dynamic behavior using the Leslie-Ericksen and Frank elastic free energy theories and assumed a homogeneous order parameter $S$. Their simulation results concluded that: (1) minimizing the Frank elastic free energy is the mechanism of mode selection between radial and onion texture, (2) the onion texture forms at high temperature when bend elastic constant $K_{3}$ is larger than splay elastic constant $K_{1}$, the radial texture forms at low temperature when bend elastic constant $K_{3}$ is smaller than splay elastic constant $K_{1}$, and (3) a random texture is created in an intermediate temperature range when elastic constants $K_{3}$ and $K_{1}$ are equal. The simulation results are shown in Figure 3.4. 


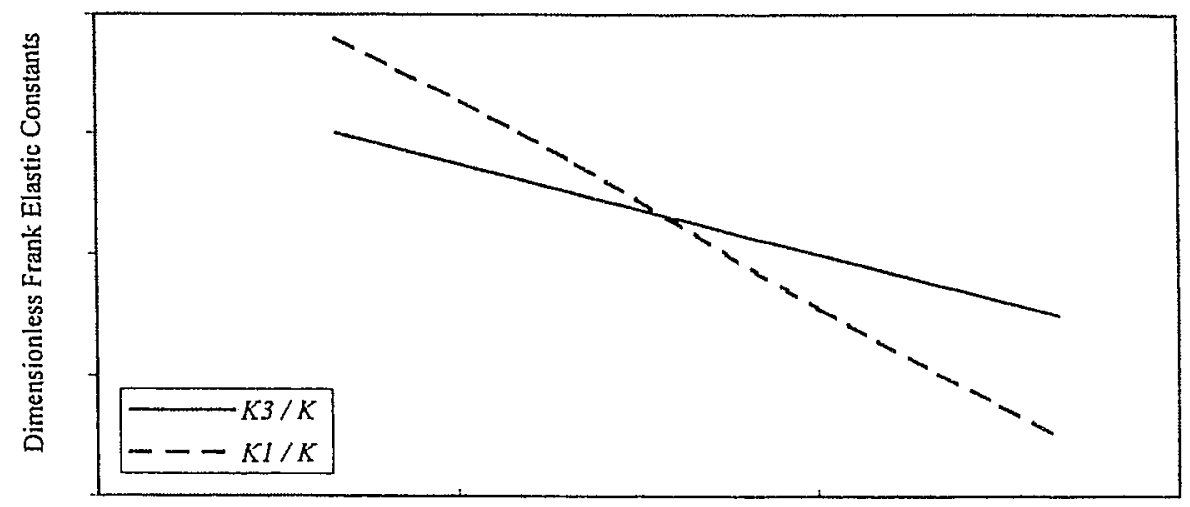

Dimensioniess Temperature $T / T_{\mathrm{NI}}$

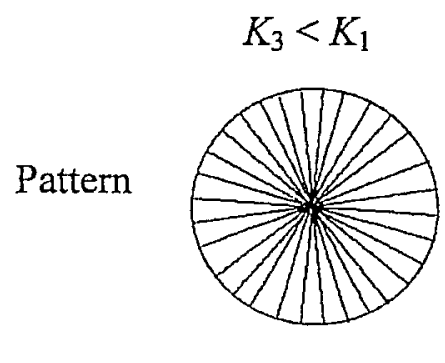

Radial

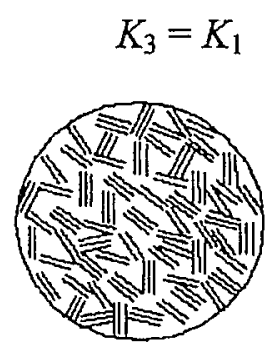

Random

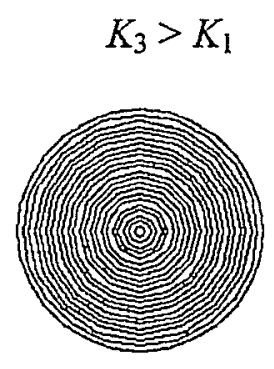

Onion

Increasing temperature

Figure 3.4 Temperature variation in elastic constants and transverse textures predicted by Wang and Rey. The temperature is scaled by the transition temperature $T_{\mathrm{NI}}$ at which $K=K_{3}=K_{1}$, and the elastic constants are scaled by $K$. The model predicted that at low temperature the bend elastic constants $K_{3}<K_{1}$, leading to a bend mode deformation, causing the pitch molecules to orient radially in fibers cross section. At high temperature, $K_{3}>K_{1}$, yielding a concentric, onion arrangement of the molecules in fibers cross-section. At an intermediate temperature range, $K=K_{3}$ $=K_{1}$, a random texture is created [50]. 


\subsubsection{McHugh and Edie's Model}

McHugh and Edie [9] modeled mesophase pitch melt flow through circular and rectangular channels to study the spinneret geometry effect on the structure formation for mesophase pitch based carbon fibers. The consistency of the model analytical results and the textures observed from experiments proved that liquid crystal theories such as the Frank elastic and Leslie-Ericksen continuum theories, developed initially for rod-like liquid crystals are applicable to describe discotic mesophase pitch molecular dynamics.

By applying mass, linear momentum and angular momentum balances using equations (2.1), (2.5), and (2.8), McHugh and Edie [9] formulated a set of equations to govern the molecular orientation behaviors in capillary and channel flows. The analytical solution of their model is, as displayed in Figure 3.5: (1) round fibers spun through a circular spinneret tend to exhibit the radial texture, and (2) ribbon-shaped fibers extruded through a rectangular spinneret tend to form line-origin textures. Later studies investigated the non-circular fiber structure and indicated that carbon fibers with this kind of texture improved the degree of liquid crystalline molecular orientation and thus have much better thermal and electrical conductivities.

The model results show that melt spinning through non-circular spinnerets offers the possibility to improve degree of orientation of mesophase pitch based carbon fibers. Studies $[16,39]$ have shown that the high degree of ordered texture is the desired structure for materials requiring excellent thermal conductivity. 

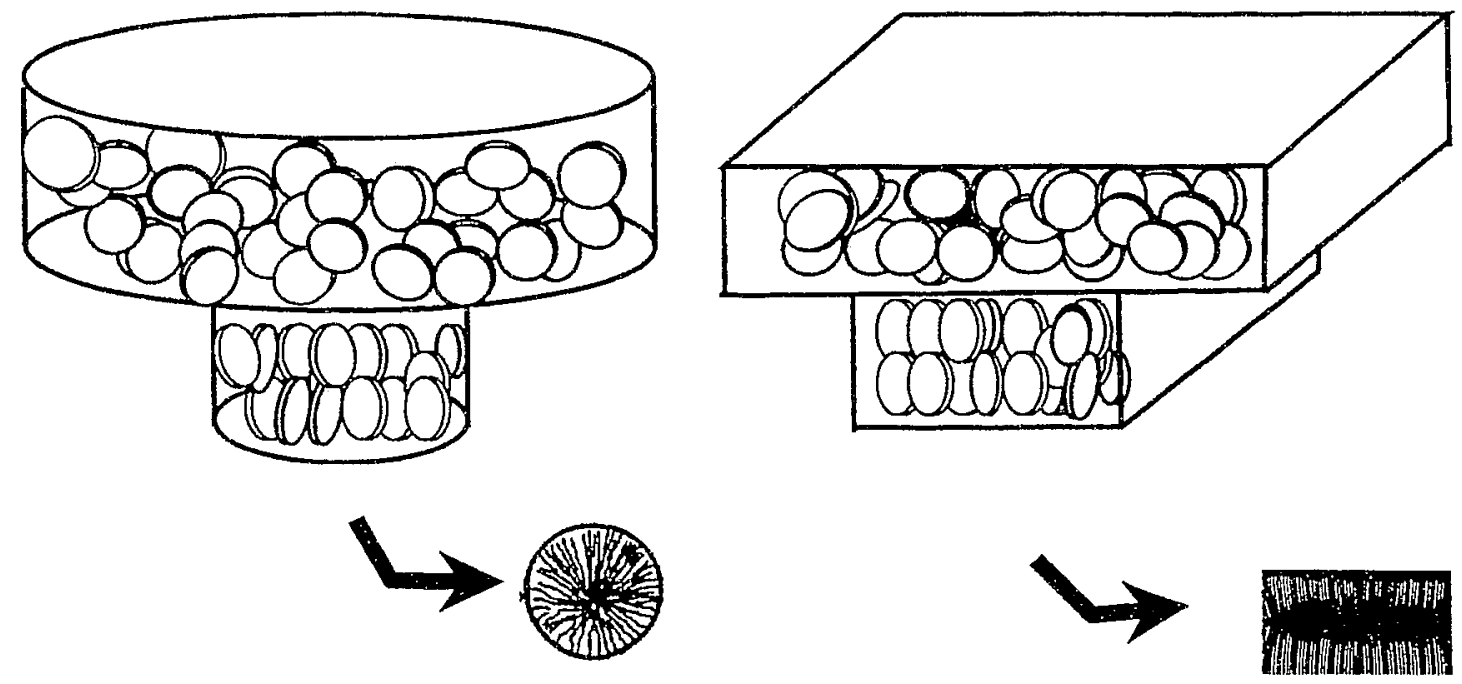

Figure 3.5 McHugh and Edie's model results. It shows that the round shape spinnerets generate a radial texture fiber; the non-circular cross sectional shape spinnerets will create a fiber with linear texture, which would have higher degree of orientation $[16,39]$.

These experimental and mathematical studies show that the textures of mesophase pitch based carbon fibers will vary with the composition of the mesophase pitch, the spinning temperature, the geometry of the orifice, etc.. In order to obtain certain product fiber properties, it is necessary to modify the pitch precursor, spinneret structure and spinning conditions such as spinning temperatures $[3,4]$. However, the degree of molecular orientation development during the melt spinning process remains less investigated, but an important issue. Since the material properties of carbon fibers depend on the molecular structure, it is crucial to investigate the structure development during the melt spinning process in order to capture the fibers degree of orientation evolution. Gallego and Edie [15] indicated current commercialized fibers possess poorly optimized molecular orientation, 
which prevents mesophase pitch-based carbon fibers usage in high performance applications. To improve the degree of the molecular orientation, the present production method is to apply high temperature graphitization, which accounts for most of the processing cost of mesophase pitch based carbon fibers. If the molecular orientation $c$ optimized during melt spinning, a lower temperature may be used to graphite the ciau fiber and eventually reduce the production cost. 


\section{Chapter 4}

\section{One-Dimensional Model Development, Results and Discussion}

\subsection{Introduction to One-Dimensional Model}

This chapter studies the relaxation phenomena and the dynamic of mesophase pitch molecules during cooling process after melt extrusion, using the more general Ericksen and Landau-de Gennes nematic continuum theories. This thermal relaxation involves heat transfer from the melt pitch to the cooling medium, which creates a radial temperature gradient across the fiber. Moreover, it is also during this thermal relaxation that the flow-induced structure starts to relax from a stressed high-energy to a low-energy state. Since the material properties of carbon fibers depend on the molecular structure, it is crucial to uncerstand the time evolution of the director and the order parameter during the thermal relaxation period.

The aim of this chapter is to investigate numerically the structure development and texture formation during the thermal relaxation after cessation of uniaxial flow for a carbonaceous mesophase pitch. Furthermore, this chapter examines specifically the effects that the melt spinning process parameters and material properties have on the time for structure development and the final texture formation. This information is important for producing a specified fiber structure with desired mechanical and transportation properties. 
The rest of the chapter consists of the development of the partial differential equations that govern the relaxation phenomena of nonhomogeneously ordered mesophase melt after extrusion flow. It also contains physical and mathematical interpretations of the simulation results, discussions on the solution of these equations under different conditions, and a section of summary and concluding remarks.

The following assumptions are used in this one-dimensional model:

(1) The mesophase pitch is thermotropic, which means that the phase transition of the melt is only affected by temperature changes.

(2) Mesophase is incompressible, i.e., the mesophase pitch density $\rho$ are constant.

(3) There are no external body forces, such as the gravitational field. Hence, $\mathbf{F}=0$.

(4) The director remains within the $x-y$ plane and there is cylindrical symmetry about $z$-axis (i.e., the fiber axis). Mesophase pitch thermal conductivity $k_{\text {th }}$ is a constant within the $x-y$ plane

(5) Backflows are negligible and neglected, and

(6) The inertia of the director is negligible and neglected.

Figure 4.1 shows a schematic representation of the disc-like molecules within a carbonaceous mesophase fiber of radius $R$, and defines the director variation angle $\theta$ within $x-y$ plane. The director field is defined as:

$$
\mathbf{n}=(\cos \theta, \sin \theta, 0)
$$

where the unit length constraint, $\mathbf{n} \cdot \mathbf{n}=1$ is automatically satisfied.

Within the planar one-dimensional approximation, the three unknowns are as follows: 


$$
T=T(x, t)
$$

$$
\theta=\theta(x, t)
$$

$$
S=S(x, t)
$$

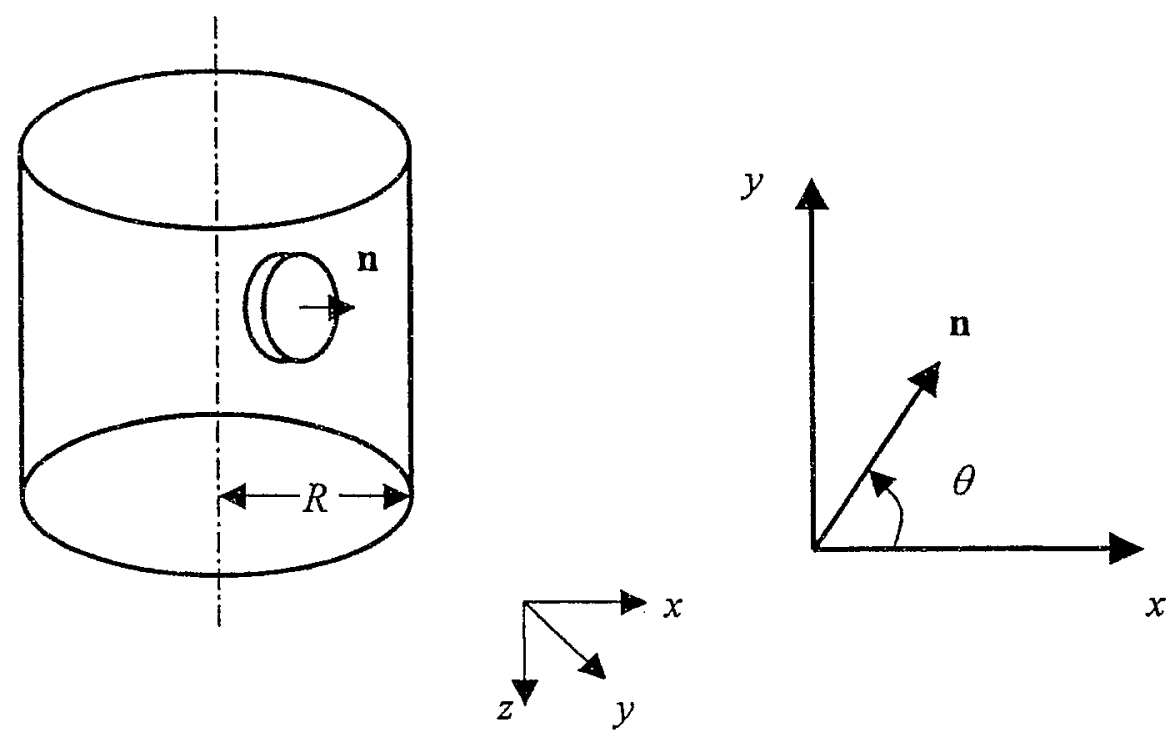

Figure 4.1 Schematic representation of the disc-like molecules within a section of a carbonaceous mesophase fiber, and the definition of the Cartesian coordinate system. $\theta$ is the planar director orientation angle measured in radians, and $R$ is the radius of the fiber. 
Since there are three unknowns, three equations are needed. The absolute temperature of the fiber $T$ can be determined by using the following unsteady heat conduction from the energy balance equation expressed as [38]:

$\rho C_{\mathrm{p}} \frac{\partial T}{\partial t}=k_{\mathrm{th}} \nabla^{2} T$

where $C_{\mathrm{p}}$ is the heat capacity of the nematic at constant pressure per unit mass, and $k_{\text {th }}$ is the thermal conductivity of the nematic phase, and $\rho$ is the nematic phase density.

Another two balance equations can be derived from the modified Ericksen theories, i.e., the $z$-component internal angular momentum balance equation, and the scalar order parameter balance equation.

\subsection{Balance Equation Formulation}

This section consists of the development of the one-dimensional partial differential equations that govern the time evolution of the director, temperature, and the scalar order parameter within the carbonaceous mesophase fiber during the cooling process after it is pulled out of the spinneret. The set of the partial differential equations then is nondimensionalized. Lastly, the auxiliary equations, which include the initial and boundary conditions, are presented.

\subsubsection{Landau-de Gennes Free Energy}

The molecular orientation is governed by the $z$-component of the internal momentum balance equation as defined by Ericksen continuum theory (see equation (2.17a)). The static energy term is defined by Landau-de Gennes energy expressed as equation (2.15). 
The coefficients $A, B$, and $C$ in the Landau-de Gennes free energy density (equation (2.15)) are not known for a nematic discotic liquid crystal. It is then convenient to replace the terms introduced by these coefficients in the free energy density by the expression given by Doi and Edwards (equation $(2,19)$ ). Then the Landau-de Gennes Free energy density can be expressed as:

$$
\begin{aligned}
f_{\mathrm{L}} & =k_{B} \cup T\left[\frac{1}{2}\left(1-\frac{1}{3} U\right) S^{2}-\frac{1}{9} U S^{3}+\frac{1}{6} U S^{4}\right] \\
& +\frac{3}{4}\left(L_{\mathrm{I}}+\frac{1}{6} L_{2}\right) \nabla S^{2}+\frac{3}{8} L_{2}(\mathbf{n} \cdot \nabla S)^{2} \\
& +\frac{9}{4} S^{2}\left[\left(L_{1}+\frac{1}{2} L_{2}\right)(\nabla \cdot \mathbf{n})^{2}+L_{1}(\mathbf{n} \cdot \nabla \times \mathbf{n})^{2}+\left(L_{1}+\frac{1}{2} L_{2}\right)(\mathbf{n} \times \nabla \times \mathbf{n})^{2}\right] \\
& +\frac{3}{2} L_{2} S(\nabla \cdot \mathbf{n}) \times(\mathbf{n} \cdot \nabla S)+\frac{3}{4} L_{2} S(\mathbf{n} \times \nabla \times \mathbf{n}) \cdot \nabla S
\end{aligned}
$$

\subsubsection{Angular Momentum Balance Equation and Scalar Order Parameter Balance Equation}

To derive the $z$-component of the internal angular momentum balance equation, assumptions given above, and equations (2.7), (2.12), (2.15), (2.17), (A.1i), and (A.1j) are used. The result of this derivation is:

$-\gamma_{1}^{S} \frac{\partial \theta}{\partial t}=-9 S\left(L_{1}+\frac{1}{2} L_{2}\right) \frac{\partial \theta}{\partial x} \frac{\partial S}{\partial x}-\frac{9}{2} S^{2}\left(L_{1}+\frac{1}{2} L_{2}\right) \frac{\partial^{2} \theta}{\partial x^{2}}+\frac{3}{8} L_{2} S \sin (2 \theta) \frac{\partial^{2} S}{\partial x^{2}}$

To derive the scalar order parameter balance equation, assumptions given above, and equations (2.12), (2.15), (2.17b), (A.1g), and (A.1h) are used. The result of this derivation is: 


$$
\begin{aligned}
-\beta_{2}^{S} \frac{\partial S}{\partial t}= & k_{B} v T\left[\left(1-\frac{T_{\mathrm{NI}}}{T}\right) S-\frac{T_{\mathrm{NI}}}{T} S^{2}+2 \frac{T_{\mathrm{Nl}}}{T} S^{3}\right] \\
& +\frac{9}{2} S\left(L_{1}+\frac{1}{2} L_{2}\right)\left(\frac{\partial \theta}{\partial x}\right)^{2}-\frac{3}{2}\left(L_{1}+\frac{1}{6} L_{2}\right) \frac{\partial^{2} S}{\partial x^{2}} \\
& +\frac{3}{4} L_{2} \sin (2 \theta) \frac{\partial \theta}{\partial x} \frac{\partial S}{\partial x}-\frac{3}{4} L_{2} \cos ^{2}(\theta) \frac{\partial^{2} S}{\partial x^{2}} \\
& +\frac{3}{4} L_{2} S \cos (2 \theta)\left(\frac{\partial \theta}{\partial x}\right)^{2}+\frac{3}{8} L_{2} S \sin (2 \theta) \frac{\partial^{2} \theta}{\partial x^{2}}
\end{aligned}
$$

Using relations derived by Beris and Edwards [26] (see equation (A.1)), the final results of these derivations are, respectively, as follows:

$$
\begin{aligned}
-\gamma_{1} \frac{\partial \theta}{\partial t}= & \kappa_{1} \frac{\partial S}{\partial x} \frac{\partial \theta}{\partial x}+\kappa_{2} \frac{\partial^{2} \theta}{\partial x^{2}}+\kappa_{3} \frac{\partial^{2} S}{\partial x^{2}} \\
-\gamma_{1} \frac{\partial S}{\partial t} & =\kappa_{\mathrm{B}} v T\left[\left(1-\frac{T_{\mathrm{NI}}}{T}\right) S-\frac{T_{\mathrm{NI}}}{T} S^{2}+2 \frac{T_{\mathrm{NI}}}{T} S^{3}\right]\left(3+6 S^{2}\right) \\
& +\kappa_{4}\left(\frac{\partial \theta}{\partial x}\right)^{2}+\kappa_{5} \frac{\partial^{2} S}{\partial x^{2}}+\kappa_{6} \frac{\partial \theta}{\partial x} \frac{\partial S}{\partial x} \\
& +\kappa_{7} \frac{\partial^{2} S}{\partial x^{2}}+\kappa_{8}\left(\frac{\partial \theta}{\partial x}\right)^{2}+\kappa_{9} \frac{\partial^{2} \theta}{\partial x^{2}}
\end{aligned}
$$

In equations (4.7) and (4.8) the elastic functions $\left\{\kappa_{i}\right\}, i=1, \ldots, 9$, are given in the Appendix. It should be noticed that the elastic functions are dependent on director orientation angle $\theta$ and the scalar order parameters $S$. In equation (4.8), $T_{\mathrm{NI}}$ is the nematic-isotropic transition temperature, and $T$ is the absolute temperature of the fiber, which can be determined by using the energy balance equation (4.3). 
The one-dimensional expression of the energy balance equation (4.3) is:

$$
\rho C_{\mathrm{p}} \frac{\partial T}{\partial t}=k_{\mathrm{th}} \frac{\partial^{2} T}{\partial x^{2}}
$$

\subsubsection{Dimensionless Equations}

The dimensionless equations are obtained by scaling the temperature with $T_{\mathrm{NI}}$, the elastic

terms with $K$, the viscosity terms with $\gamma_{1}$, the length with $R$, and the time with $\frac{\gamma_{1} R^{2}}{K}$.

By doing this, the elastic functions become $\kappa_{i}^{*}=\kappa_{i} / K$. The superscript asterisk denotes a dimensionless variable. The equations then become the following set of dimensionless non-linear partial differential equations:

$$
\begin{aligned}
\frac{\partial T^{*}}{\partial t^{*}} & =\alpha \frac{\partial^{2} T^{*}}{\partial x^{* 2}} \\
-\frac{\partial \theta}{\partial t^{*}} & =\kappa_{1}^{*} \frac{\partial S}{\partial x^{*}} \frac{\partial \theta}{\partial x^{*}}+\kappa_{2}^{*} \frac{\partial^{2} \theta}{\partial x^{* 2}}+\kappa_{3}^{*} \frac{\partial^{2} S}{\partial x^{* 2}} \\
-\frac{\partial S}{\partial t^{*}} & =\beta\left[\left(T^{*}-1\right) S-S^{2}+2 S^{3}\right]\left(3+6 S^{2}\right)+\kappa_{4}^{*}\left(\frac{\partial \theta}{\partial x^{*}}\right)^{2}+\kappa_{5}^{*} \frac{\partial^{2} S}{\partial x^{* 2}} \\
& +\kappa_{6}^{*} \frac{\partial \theta}{\partial x^{*}} \frac{\partial S}{\partial x^{*}}+\kappa_{7}^{*} \frac{\partial^{2} S}{\partial x^{* 2}}+\kappa_{8}^{*}\left(\frac{\partial \theta}{\partial x^{*}}\right)^{2}+\kappa_{9}^{*} \frac{\partial^{2} \theta}{\partial x^{* 2}}
\end{aligned}
$$

Equation (4.10) introduces a dimensionless thermal diffusivity $\alpha$ and a dimensionless characteristic molecular free energy $\beta$, which are expressed as follow: 
$\alpha=\frac{k_{\mathrm{th}}}{\rho C_{\mathrm{p}}} \frac{\gamma_{1}}{K}$

$\beta=\frac{k_{\mathrm{B}} \nu T_{\mathrm{N}} R^{2}}{K}$

The dimensionless thermal diffusivity $\alpha$ depends on both the melt transport properties and the liquid crystalline properties. In this study, we use the range $1 \leq \alpha \leq 10^{5} . \quad \beta$ is defined as the ratio of short-range order elasticity to long-range order elasticity [20], and in this study we use $\beta=10^{4}$ to be consistent with prior published theoretical work [20-23]. Furthermore, these values allow us to obtain the objectives of the current study.

The material physical properties and the melt spinning process parameters used in this model are tabulated in Tables 4.1 to 4.3 . The values for $K, K_{5}$, and $K_{6}$ are obtained by assuming a dimensionless ratio of $L_{2}^{*}=\frac{L_{2}}{L_{1}}$. The rotational viscosity of the mesophase pitch melt is assumed as $\gamma_{1}=1.0 \mathrm{~Pa} \cdot \mathrm{S}$ [48]. The values of $L_{2}^{*}$ and $\gamma_{1}$ are in the same range as published theoretical work $[20-23,48]$, since no experimentally determined values can be found for them. The three elastic constants are of the order of magnitude of $10^{-12} \mathrm{~N}$ [24]. Furthermore, the elastic constants fulfill the constitutive hypothesis set by the following conditions [34]:

$L_{1} \geq 0$ and $L_{1}+\frac{2}{3} L_{2} \geq 0$

Lastly, according to equation $(2.16 \mathrm{~b}, \mathrm{c}), L_{2}<0$ is required for discotic liquid crystals. 
Table 4.1 Elastic constants

\begin{tabular}{|l|l|}
\hline$K=9 L_{1}+\frac{9}{2} L_{2}$ & $30.0 \times 10^{-12} \mathrm{~N}$ \\
\hline$K_{5}=\frac{3}{2} L_{1}+\frac{1}{4} L_{2}$ & $6.0 \times 10^{-12} \mathrm{~N}$ \\
\hline$K_{6}=\frac{3}{4} L_{2}$ & $-1.5 \times 10^{-12} \mathrm{~N}$ \\
\hline$L_{2}^{*}=\frac{L_{2}}{L_{1}}$ & $-\frac{6}{13}$ \\
\hline
\end{tabular}

Table 4.2 Melt spinning parameters

\begin{tabular}{|l|l|}
\hline$T_{\mathrm{S}} \quad$ (fiber spinning temperature) & $600 \mathrm{~K}[1]$ \\
\hline$T_{\mathrm{C}} \quad$ (cooling air temperature) & $373 \mathrm{~K}[1]$ \\
\hline$T_{\mathrm{NI}} \quad$ (nematic-isotropic transition temperature) & $725 \mathrm{~K}[10]$ \\
\hline
\end{tabular}

Table 4.3 Physical properties for carbon fibers

\begin{tabular}{|l|l|}
\hline$R$ (fiber radius) & $5.5 \times 10^{-6} \mathrm{~m}[1]$ \\
\hline$\rho$ (mesophase pitch density) & $2000 \mathrm{~kg} / \mathrm{m}^{3}[1]$ \\
\hline$C_{\mathrm{p}}$ (heat capacity) & $1000 \mathrm{~J} /(\mathrm{kg} \cdot \mathrm{K})[3]$ \\
\hline$k_{\mathrm{th}} \quad$ (thermal conductivity) & $7.5 \mathrm{~W} /(\mathrm{m} \cdot \mathrm{K})[4]$ \\
\hline$\gamma_{1} \quad$ (rotational viscosity) & $1.0 \mathrm{~Pa} \cdot \mathrm{S}[48]$ \\
\hline
\end{tabular}




\subsubsection{Auxiliary Equations}

In this one-dimensional study, the initial conditions are as follows:

$T_{i}=T_{\mathrm{S}}$ at $t=0,0 \leq x \leq R$

$\theta_{1}=\theta_{0}+\eta \varepsilon$ at $t=0,0 \leq x \leq R$

$S_{i}=0.25+0.75 \sqrt{1-\frac{8 T_{\mathrm{S}}}{9 T_{\mathrm{N}}}}$ at $t=0,0 \leq x \leq R$

where $T_{\mathrm{S}}$ is the fiber spinning temperature, $T_{\mathrm{C}}$ is the cooling air temperature, and $T_{\mathrm{NI}}$ is the nematic-isotropic phase transition temperature. Values for these temperatures are listed in Table 4.2.

The initial director field is assumed to be random as given by equation (4.14b), even though during the uniaxial elongation flow the spinnerate is supposed to align the discotic molecules along the flow direction. In equation (4.14b), $\varepsilon$ is a random number determined using a standard random number generator and is within the range $0<\varepsilon<1$, and $\eta$ is a factor that controls the magnitude of the fluctuation. In this study, $\eta=1.0$ is used. The choice of the algebraic operation \pm is determined randomly by using a random number generator with a different seed than that used to generate $\varepsilon$. If the random number generated is less than 0.5 , the sign is negative; otherwise, it is positive.

The boundary conditions are as follows: 


$$
\begin{aligned}
& \frac{\partial T}{\partial x}=0 \text { at } t>0, x=0 \\
& T=T_{\mathrm{C}} \text { at } t>0, x=R \\
& \frac{\partial \theta}{\partial x}=0 \text { at } t>0, x=0 \\
& \theta=0 \text { at } t>0, x=R \\
& \frac{\partial S}{\partial x}=0 \text { at } t>0, x=0 \\
& S=0.25+0.75 \sqrt{1-\frac{8 T_{\mathrm{C}}}{9 T_{\mathrm{N}}}} \text { at } t>0, x=R
\end{aligned}
$$

$$
S_{i}=0.25+0.75 \sqrt{1-\frac{8 T_{\mathrm{S}}}{9 T_{\mathrm{NI}}}} \text { at } t^{*}=0,0 \leq x^{*} \leq 1
$$


$\frac{\partial T^{*}}{\partial x^{*}}=0$ at $t^{*}>0, x^{*}=0$

$T^{*}=T_{\mathrm{c}} / T_{\mathrm{N} 1}$ at $t^{*}>0, x^{*}=1$

$\frac{\partial \theta}{\partial x^{*}}=0$ at $t^{*}>0, x^{*}=0$

$\theta=0$ at $t^{*}>0, x^{*}=1$

$\frac{\partial S}{\partial x^{*}}=0$ at $t^{*}>0, x^{*}=0$

$S=0.25+0.75 \sqrt{1-\frac{8 T_{\mathrm{C}}}{9 T_{\mathrm{N} I}}}$ at $t^{*}>0, x^{*}=1$

\subsection{Method of Solutions}

In summary, in the one-dimension model the dependent variables are dimensionless temperature $T^{*}$, the scalar order parameter $S$ and director orientation $\theta$. The independent variables are the dimensionless length $x^{*}$ and dimensionless time $t^{*}$. Equations $(4.10 \mathrm{a}-\mathrm{c})$ are solved numerically with the dimensionless initial and boundary conditions given by equations $(4.15 \mathrm{a}-\mathrm{i})$. The Galerkin finite element method is used with 20 linear elements. The time integrator is the first order Euler predicator-corrector method, and the Newton-Raphson method is used for solving the system of non-linear algebraic equations. Convergence is assumed when the length of the vector is less than $10^{-6}$. 


\subsection{Results and Discussion}

This section presents and discusses representative numerical solutions to equations $(4.10 \mathrm{a}-\mathrm{c})$ and $(4.15 \mathrm{a}-\mathrm{i})$. It begins by showing the time evolution of the molecular field, and temperature and the scalar order parameter spatial profiles for $\alpha=10^{2}$ and $\beta=10^{4}$. This is followed by a discussion on the effect of the dimensionless thermal diffusivity $\alpha$ on the thermal relaxation phenomenon. This section concludes with a discussion on how the dimensionless thermal diffusivity $\alpha$ affects the order development and texture formation in carbon fibers.

Figure 4.2 shows the one-dimensional relaxation phenomena of the molecular field for the case when $\alpha=10^{2}$ and $\beta=10^{4}$ at the following dimensionless times $t^{*}$ : (a) 0.0 , (b) 0.001 , (c) 0.002 , (d) 0.006 , and (e) 0.05 . The short line segments represent the edges of the discotic molecules, and are obtained by noting that a director is normal to the disc-like molecule. The plot shows that the molecules go from a randomly aligned state obtained using a random number generator to a partially aligned state, and finally evolve into a perfect aligned structure with minimal distortion Frank elastic free energy $F_{\mathrm{d}}{ }^{*}$. This is shown in Figure 4.3. 
(a)

$\lambda \times \Lambda / 1 \times \times 1+k / x=1$

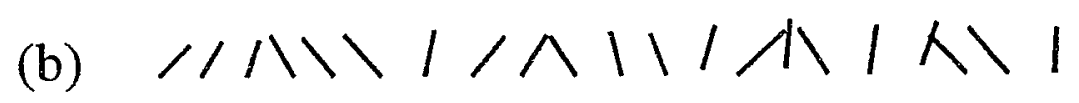

(c) $1 / 1 / 1 / 1 / 1 / 1 / 1 / 111111$

(d)

111111111111111111111

$\checkmark(\mathrm{e})$

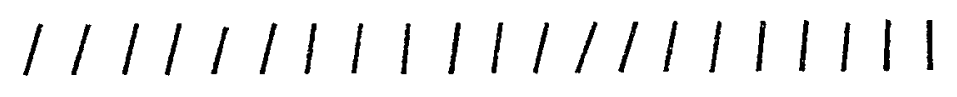

Figure 4.2 Typical relaxation phenomena for molecular orientation for the case $\alpha=10^{2}$ and $\beta=10^{4}$, in fiber radial direction at the following dimensionless times $t^{*}$ : (a) 0.0 , (b) 0.001 , (c) 0.002 , (d) 0.006 , and (e) 0.05 . 
Figure 4.3 is a plot of the stored Frank elastic free energy $F_{\mathrm{d}}{ }^{*}$ versus dimensionless time $t^{*}$. The one dimensional dimensionless form $F_{\mathrm{d}}{ }^{*}$ may be obtained using equation (2.2) and is expressed as:

$$
F_{\mathrm{d}}^{*}=\frac{1}{4} \int_{b}^{1} S^{2}\left(\frac{\partial \theta}{\partial x^{*}}\right)^{2} d x^{*}
$$

In order to characterize the time evolution of the molecular reorientation from the totally random oriented state to the aligned state, in this one-dimension model, the time $\tau_{\mathrm{d}}$ and $\tau_{0}$ is defined as the time required for the molecules to reorient such that the Frank elastic free energy $F_{d}{ }^{*}$ decreases by $86.5 \%$ and $98 \%$, respectively. For this case, $\tau_{d}=0.002$ and $\tau_{0}=0.006$. This figure shows that Frank elastic free energy $F_{\mathrm{d}}{ }^{*}$ evolution goes through three stages: fast, intermediate, and weak relaxation. The three relaxation stages are consistent with the texture development exhibited in Figure 4.2. In the fast relaxation stage, corresponding to $t^{*}<0.002$ (or $\tau_{\mathrm{d}}$ ) in Figure 4.2 , the molecules are randomly oriented leading to a strained texture. This initial texture stores the greatest-level distortion free energy, which is the driving force for the fast relaxation rate for $t^{*}<\tau_{\mathrm{d}}$. In stage 2, corresponding to 0.002 (or $\tau_{\mathrm{d}}$ ) $<t^{*}<0.006$ (or $\tau_{0}$ ) in Figure 4.2, the Frank free energy ${F_{\mathrm{d}}}^{*}$ decreases to $2 \%$ of its initial value, and the molecules in this period are almost perfectly aligned. In the final weak relaxation stage, corresponding to $t^{*}>0.006$ (or $\tau_{0}$ ) in Figure 4.2 , the residue of the Frank elastic free energy $F_{\mathrm{d}}{ }^{*}$ is the smaller driving force to reorient the molecules into a perfectly aligned structure.

In summary, the director relaxation phenomena can be well explained using the Frank elastic free energy theory. Discotic nematics are elastic materials, where energy is stored by orientation strains. The driving force for the texture developing from a randomly aligned texture to a perfectly aligned texture is the minimization of the stored 
Frank elastic free energy. The fastest route to minimize the stored elastic free energy is to reorient the molecules to a uniform direction.

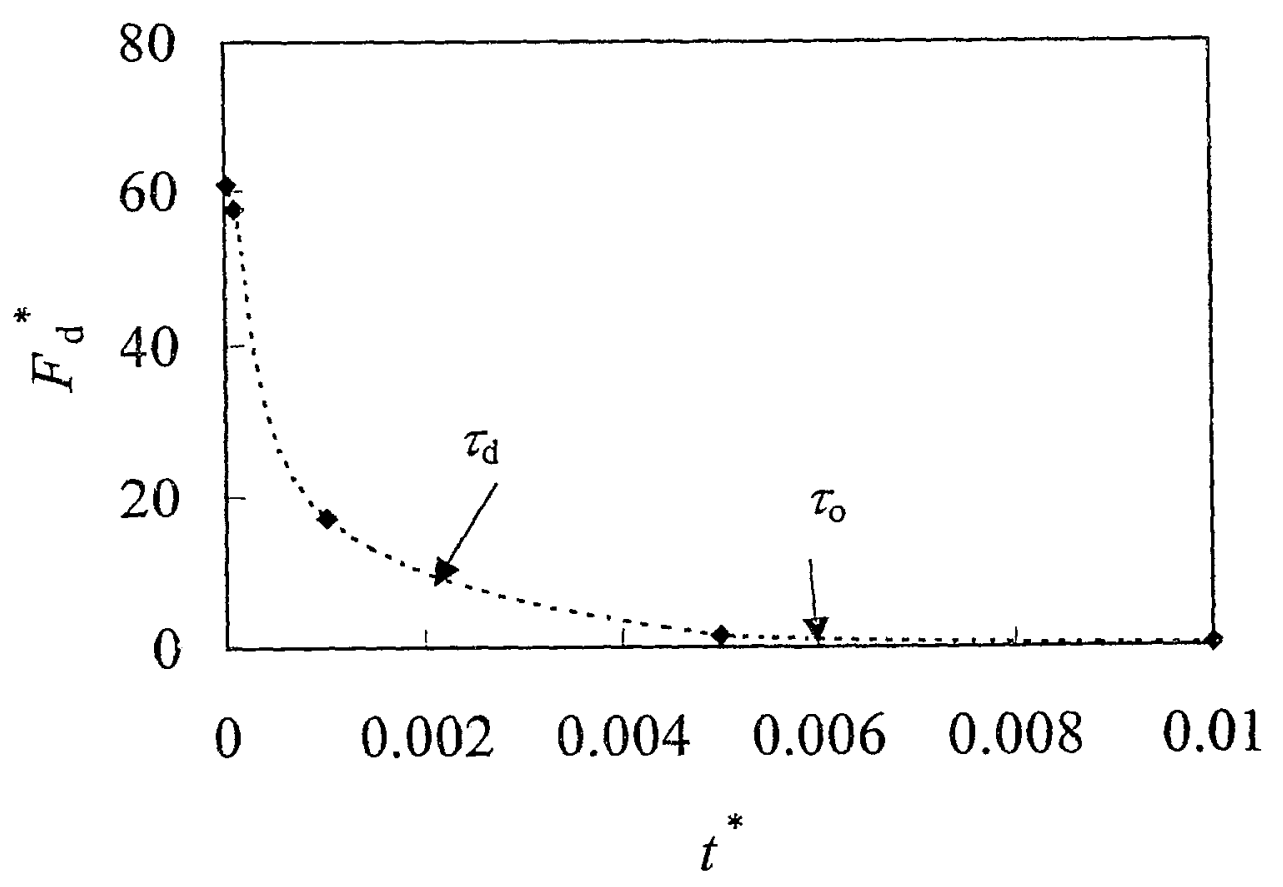

Figure 4.3 Time evolution of the dimensionless Frank elastic free energy $F_{\mathrm{d}}^{*}$ for the case $\alpha=10^{2}$ and $\beta=10^{4}, \tau_{\mathrm{d}}$ and $\tau_{\mathrm{o}}$ are defined as the time required for the molecules to reorient such that the Frank elastic free energy $F_{\mathrm{d}}{ }^{*}$ decreases by $86.5 \%$ and $98 \%$, respectively 
Figure 4.4 shows the one-dimensional relaxation phenomena of the temperature and the scalar order parameter for the case when $\alpha=10^{2}$ and $\beta=10^{4}$ at the following dimensionless times $t^{*}: 0.0001$ (diamond), 0.001 (square), 0.005 (triangle), and 0.05 (circle). Figure 4.4a shows typical thermal diffusion phenomena along the fiber radial direction. The fiber exists the spinneret at spinning temperature $T_{\mathrm{S}}=600 \mathrm{~K}$, and is suddenly exposed to cooling air temperature of $T_{\mathrm{C}}=373 \mathrm{~K}$. It is assumed that there is perfect thermal contact between the cooling air and the mesophase pitch. As expected, the temperature profile and the scalar order parameter evolution become uniform at long time (i.e., steady state).

Figure 4.4 b shows that the scalar order parameter $S$ evolution is consistent with that of the temperature profile. This may be explained using the following temperature dependency of the scalar order parameter at homogeneous equilibrium:

$S_{\mathrm{eq}}=0.25+0.75 \sqrt{1-\frac{8}{3 U}}$

where $U=3 T_{\mathrm{NI}} / T$. As mentioned in Chapter 2, for an isotropic phase $U<8 / 3$, while for a nematic phase $U>3$ [35]. For this model, nematic potential $U=3 T_{\mathrm{NI}} / T$ is inhomogeneous due to the temperature gradient across the fiber radius. The scalar order parameter $S$ is generally increasing reciprocally with temperature. 


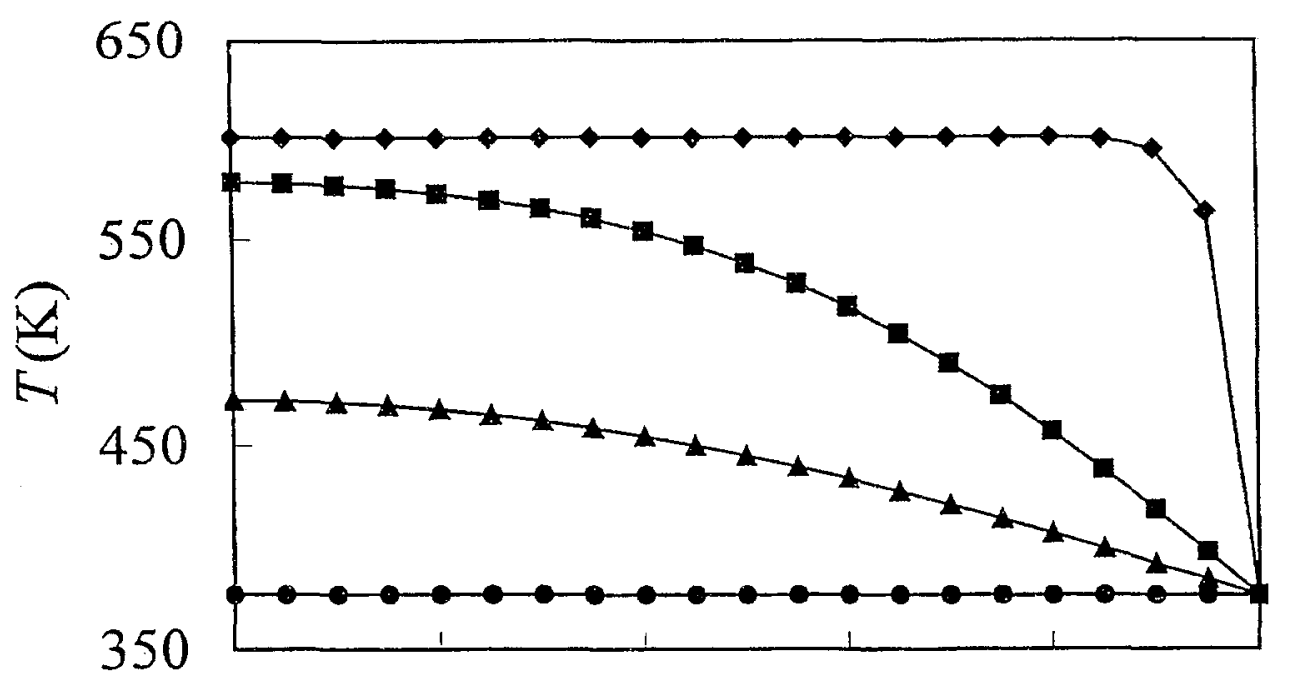

(a)

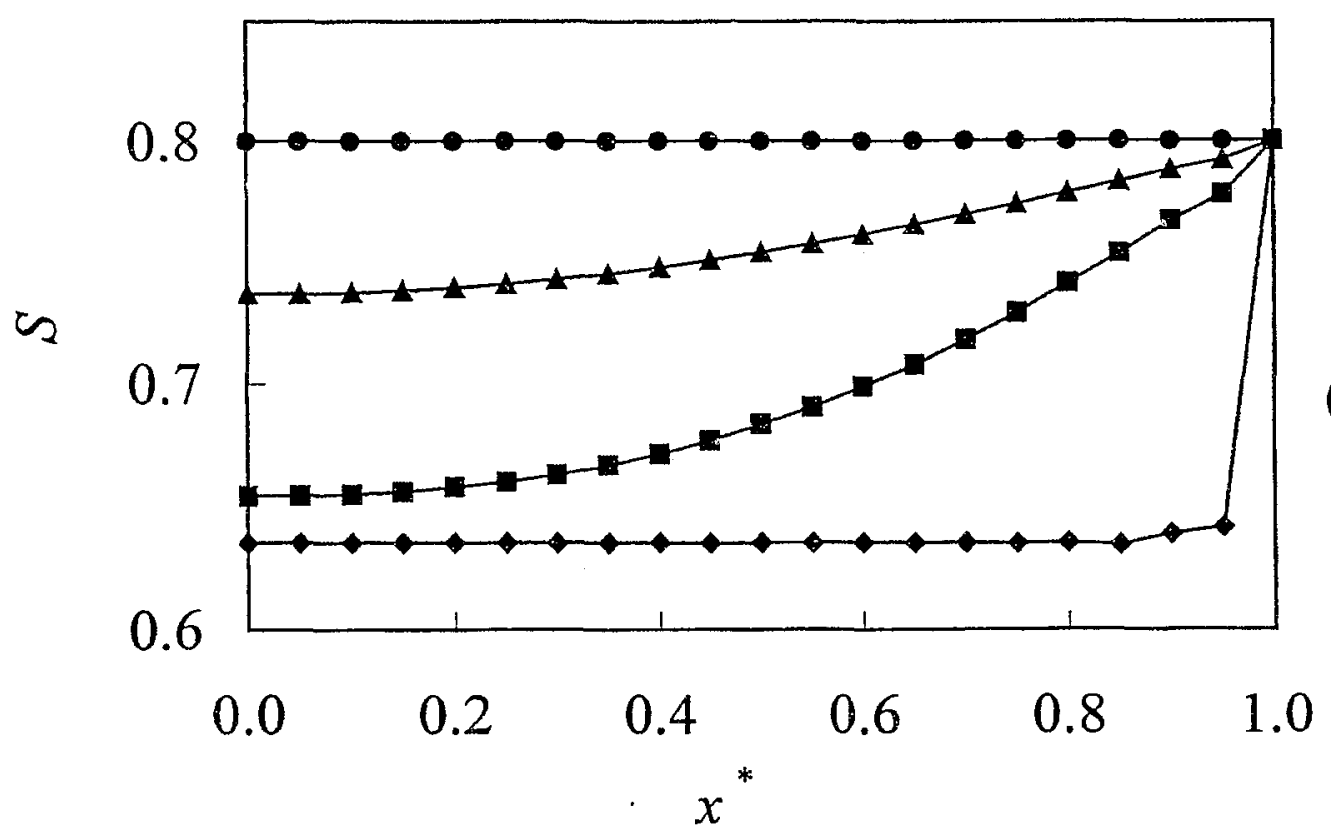

(b)

Figure 4.4 Typical relaxation phenomena for (a) the temperature profile and (b) the scalar order parameter profile in fiber radial direction for the case $\alpha=10^{2}$ and $\beta=10^{4}$ at the following dimensionless times $t^{*}: 0.0001$ (diamond), 0.001 (square), 0.005 (triangle), and 0.05 (circle). 
Figure 4.5 is a plot of the time evolution of the molecular free energy $F_{\mathrm{S}}{ }^{*}$ for the case when $\alpha=10^{2}$ and $\beta=10^{4}$, which was calculated by integrating equation (2.19) over the fiber radius. Figure 4.5 indicates that $F_{\mathrm{S}}{ }^{*}$ decreases with time, and reaches steady state at the same time as the spatial profiles of temperature and the scalar order parameter (see Figure 4.4). The time when $F_{\mathrm{S}}{ }^{*}$ decreases to the equilibrium value is denoted as the thermal relaxation process steady state time $\tau_{\alpha}$. For this case, the plot shows $\tau_{\alpha}=0.05$, and is consistent with the time required for temperature $T$ and the scalar order parameter $S$ reach steady state in figure 4.4 .

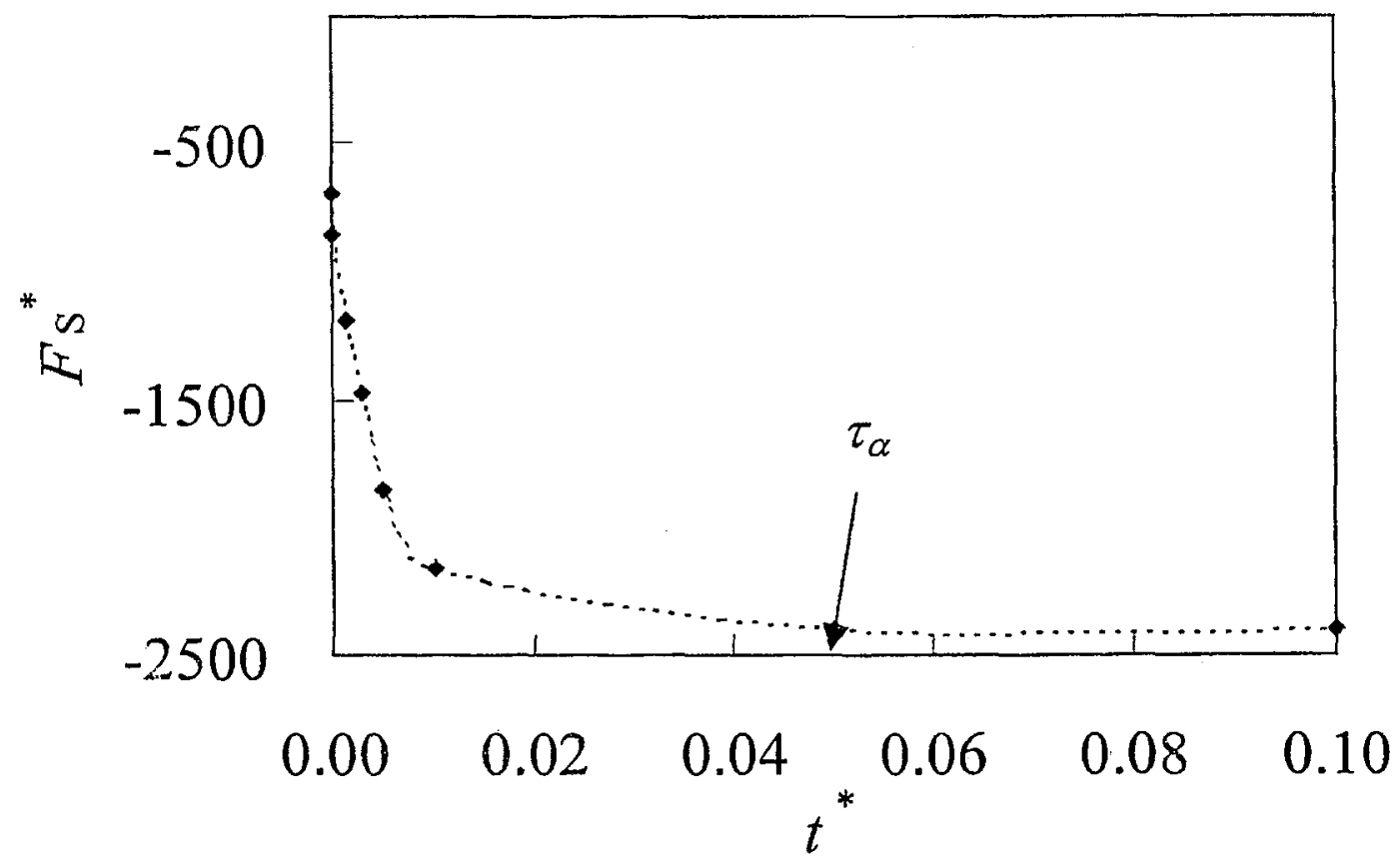

Figure 4.5 Time evolution of the dimensionless molecular free energy $F_{S}^{*}$ for the case $\alpha=10^{2}$ and $\beta=10^{4} . \quad \tau_{\alpha}$ is the time for the molecular free energy to reach steady state. 
Figure 4.6 is a plot of the dimensionless process time $\tau_{\mathrm{d}}, \tau_{\mathrm{o}}$ and $\tau_{\alpha}$ versus thermal diffusivity $\alpha$. This figure is used to discuss the effect of the dimensionless thermal diffusivity $\alpha$ on mesophasf; pitch based carbon fiber texture formation and ordered structure development. The slanted dashed line indicates that the thermal relaxation process steady state time $\tau_{\alpha}$ decreases monotonically with dimensionless thermal diffusivity $\alpha$. This means that the time for $S$ and $T$ to reach steady state decreases as $\alpha$ increases. The reason for this phenomenon is that the dimensionless thermal diffusivity is the factor that controls the rate of the heat transfer between the mesophase pitch and the cooling air (see equation (4.10a)), i.e., the rate of heat transfer increases with $\alpha$. It should be noticed that this dimensionless thermal diffusivity is also a criteria of the mesophase viscosity and elastic property. Consequently, the pitch viscoelastic property and the thermal transport property are both the important factor that controls the thermal relaxation time evolution phenomena. The two solid near-horizontal lines represent the dependencies of the dimensionless director reorientation times $\tau_{0}$ and $\tau_{\mathrm{d}}$ versus the dimensionless thermal diffusivity $\alpha$. The nearly flat lines show that the dimensionless thermal diffusivity $\alpha$ has little effect on the director field relaxation. The reason for this fact is that the relaxation rate of $F_{\mathrm{d}}{ }^{*}$ is a function of elastic constants and not a function of $\alpha$. The dynamic behaviour of the director reorientation is governed by equation ( $4.10 \mathrm{~b})$. The right side of equation ( $4.10 \mathrm{~b}$ ) has three terms, which are obtained, respectively, from $f_{\mathrm{e}}^{*}, f_{\mathrm{d}}^{*}$, and $f_{\mathrm{C}}^{*}$. The simulation results indicated that compared with Frank elastic free energy, $f_{\mathrm{e}}^{*}$ and $f_{\mathrm{C}}{ }^{*}$ induced by interaction between $S$ and $\mathbf{n}$ spatial gradients are negligible. Consequently, the time evolution of the director field in one-dimension can be expressed as:

$-\frac{\partial \theta}{\partial t^{*}}=\kappa_{2}^{*}\left(\frac{\partial^{2} \theta}{\partial x^{* 2}}\right)$ 
Equation (4.18) shows that the relaxation of director field is not affected by the thermal diffusivity because the dimensionless elastic function $\kappa_{2}^{*}$ is related only to Landau elastic constants (see equation (A.2b)). The other two terms of equation (4.10b) have little effect on the director time evolution. This leads to the director reorientation behaviour being nearly independent of the field of the scalar order parameter $S$ and temperature $T$.

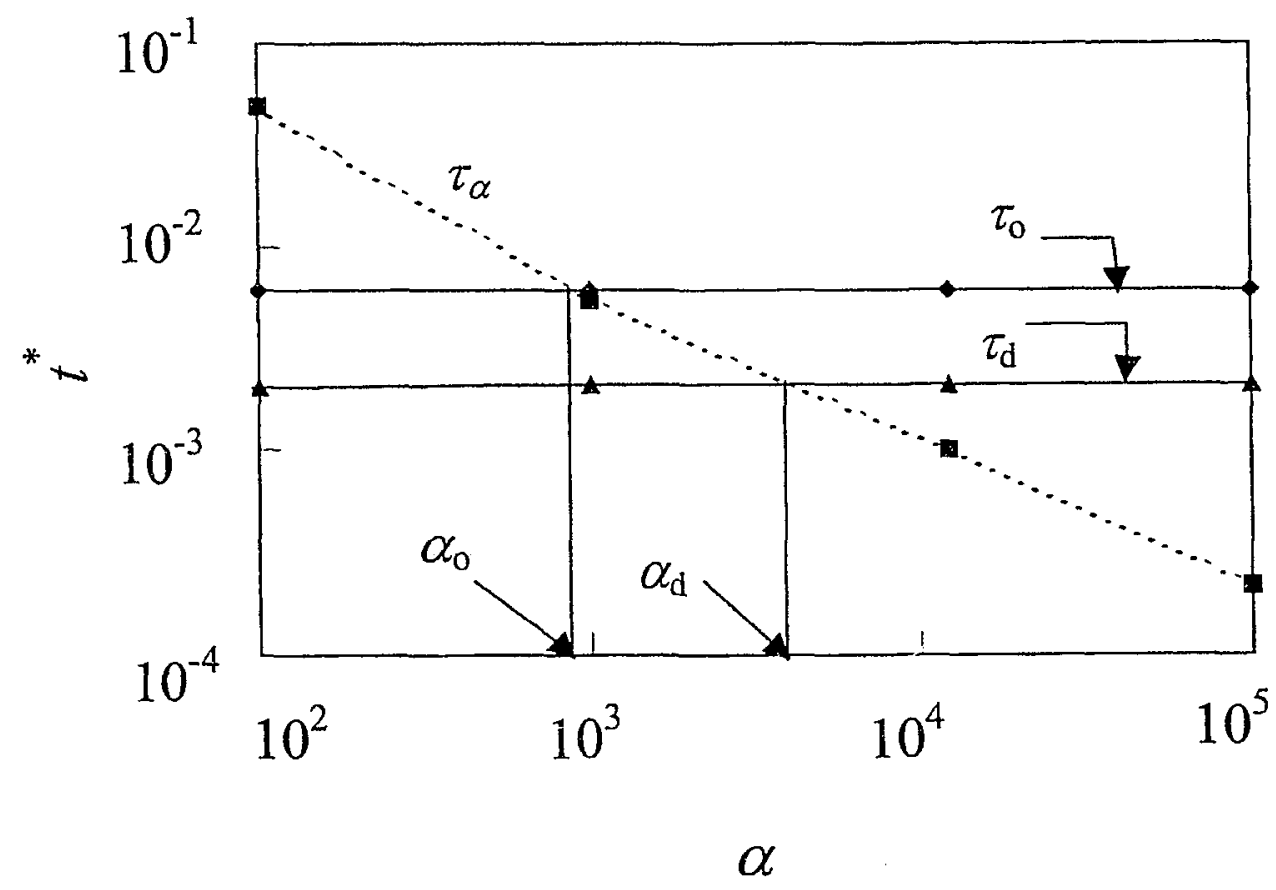

Figure 4.6 Thermal diffusivity $\alpha$ effect on the thermal relaxation. The dashed line represents the thermal relaxation time scale versus $\alpha$. The two solid lines represent the molecule reorientation time scales versus $\alpha . \alpha_{0}$ is defined as the cross point between $\tau_{\mathrm{o}}$ and $\tau_{\alpha}$, and $\alpha_{\mathrm{d}}$ is the cross point between $\tau_{\mathrm{d}}$ and $\tau_{\alpha}$. 
To characterize the thermal diffusivity effect on the structure development and texture formation, this model defines $\alpha_{0}$ as the value of $\alpha$ at the cross over point between $\tau_{\alpha}$ and $\tau_{0}$, and the value at the cross over point between $\tau_{\alpha}$ and $\tau_{\mathrm{d}}$ is denoted as $\alpha_{\mathrm{d}}$. As discussed above, $\tau_{\alpha}$ may be taken as the time for the temperature $T$ and the scalar order parameter profile to reach steady state. Furthermore, $\tau_{0}$ and $\tau_{d}$ may be taken as the time for the liquid crystal molecule spatial profile to reorient to within $13.5 \%$ and $2 \%$, respectively, of its steady state profile. High value of $\alpha$ indicates higher viscosity, lower elasticity and fast cooling rate; low value of $\alpha$ represents lower viscosity, higher elasticity and slow cooling process.

For $\alpha>\alpha_{\mathrm{d}}$, the plot shows process time $\tau_{\alpha}$ is less than reorientation time $\tau_{\mathrm{d}}$ and $\tau_{0}$. This means there is little time available for discotic molecules to rotate. This leads to a partially aligned texture depicted in Figure (4.2b). For $\alpha_{0}<\alpha<\alpha_{\mathrm{d}}$, Figure 4.6 indicates $\tau_{\mathrm{d}}<\tau_{\alpha}<\tau_{0}$. When $\alpha$ is between these values, the molecules will have some time to reorient into a highly aligned texture exhibited in Figure (4.2c). For $\alpha<\alpha_{0}$, the plot shows $\tau_{\alpha}>\tau_{\mathrm{d}}$ and $\tau_{\mathrm{o}}$. This means the reorientation time provided by the thermal relaxation process for molecules to rotate is longer than the molecules need to reorient to a perfect order structure. When the thermal diffusivity is smaller than $\alpha_{0}$, the molecules will reorient into a perfectly ordered structure as exhibited in Figure (4.2e).

\subsection{Summary}

The Ericksen and Landau-de Gennes continuum theories were used in this one-dimensional model to study numerically the structure development and texture formation in a discotic uniaxial nematic mesophase pitch based carbon fiber after cessation of extensional flow. The results indicate that the cooling step involves the relaxation of Frank elastic free energy $F_{d}{ }^{*}$ (taking into account director distortion) and the 
relaxation of molecular free energy $F_{\mathrm{S}}{ }^{*}$ expressed as a function of temperature and scalar order parameter. High thermal diffusivity caused by higher viscosity and fast cooling inhibits the liquid crystal molecular reorientation and limits the texture development. Lower viscosity and slow cooling provides more time for liquid crystal molecular reorientation and allows fiber cross sectional structure to develop into a well-defined texture.

The simulation results are consistent with White and Buechler's prediction [14]. That is the radial texture is formed at low viscosity and slow cooling, the random texture results from high viscosity and rapid quenching, and intermediate textures result from partial annihilation of declinations. These simulation results provide additional information on molecular orientation during post melt spinning process, which helps to improve the product properties and to reduce the post treatment process cost. 


\section{Chapter 5}

\section{Two-Dimensional Model Development, Results and}

\section{Discussion}

Chapter 4 presents the one-dimensional modeling and simulation of the texture formation for mesophase pitch based carbon fibers. It is capable of capturing the structure development in a nonisothermal system where the scalar order parameter and temperature is nonhomogeneous across the fiber radius. The transverse texture formation, however, is better to describe in a two-dimensional space. This chapter is dedicated to developing and simulation of texture formation for mesophase pitch based carbon fibers using the Erickesen and Landau-de Gennes continuum theories in a two-dimension space. In addition, this chapter also examines specifically the effects that the melt spinning process parameters and material properties, such as liquid crystal surface anchoring behavior and pitch melt textures, on the structure evolution and the final texture formation. This information is important for producing a specified fiber structure with desired mechanical and transportation properties.

The rest of the chapter consists of the development of the partial differential equations that govern the relaxation phenomena of nonhomogeneously ordered mesophase melt after extrusion flow in a two-dimensional space. It also contains physical and mathematical interpretations of the results, discussions on the solutions of these equations, comparison marks with one-dimensional results, and a section of summary.

The following assumptions are used in this two-dimensional study:

(1) The mesophase pitch is thermotropic. The phase transition is only effected by temperature changes. 
(2) Mesophase is incompressible, i.e., the mesophase pitch density $\rho$ is a constant.

(3) There are no external body forces, such as the gravitational field. Hence, $\mathbf{F}=0$.

(4) The director remains within the $x-y$ plane. Mesophase pitch thermal conductivity $k_{\text {th }}$ is a constant within the $x-y$ plane.

(5) Backflows are negligible.

(6) The inertia of the director is negligible and neglected.

\subsection{Introduction to Two-Dimensional Model}

Figure 5.1 shows a schematic representation of the disc-like molecules within a section of a carbonaceous mesophase fiber of radius $R$, and the definition of the coordinate systems. The polar coordinates $r-\varphi$ are expressed by dashed lines. The Cartesian coordinates $x-y$ are expressed by solid lines. $\theta$ is the planar director orientation angle measured in radians, which is the angle between director $\mathbf{n}$ and polar $r$ at point $(x, y)$.

In Cartesian system, $\theta$ is within $x-y$ plane. The director field is defined as:

$\mathbf{n}=(\cos \theta, \sin \theta, 0)$

where the unit length constraint, $\mathbf{n} \cdot \mathbf{n}=1$ is satisfied.

Within the planar two-dimensional approximation, the three unknowns are as follows:

$$
\begin{aligned}
& T=T(x, y, t) \\
& \theta=\theta(x, y, t) \\
& S=S(x, y, t)
\end{aligned}
$$



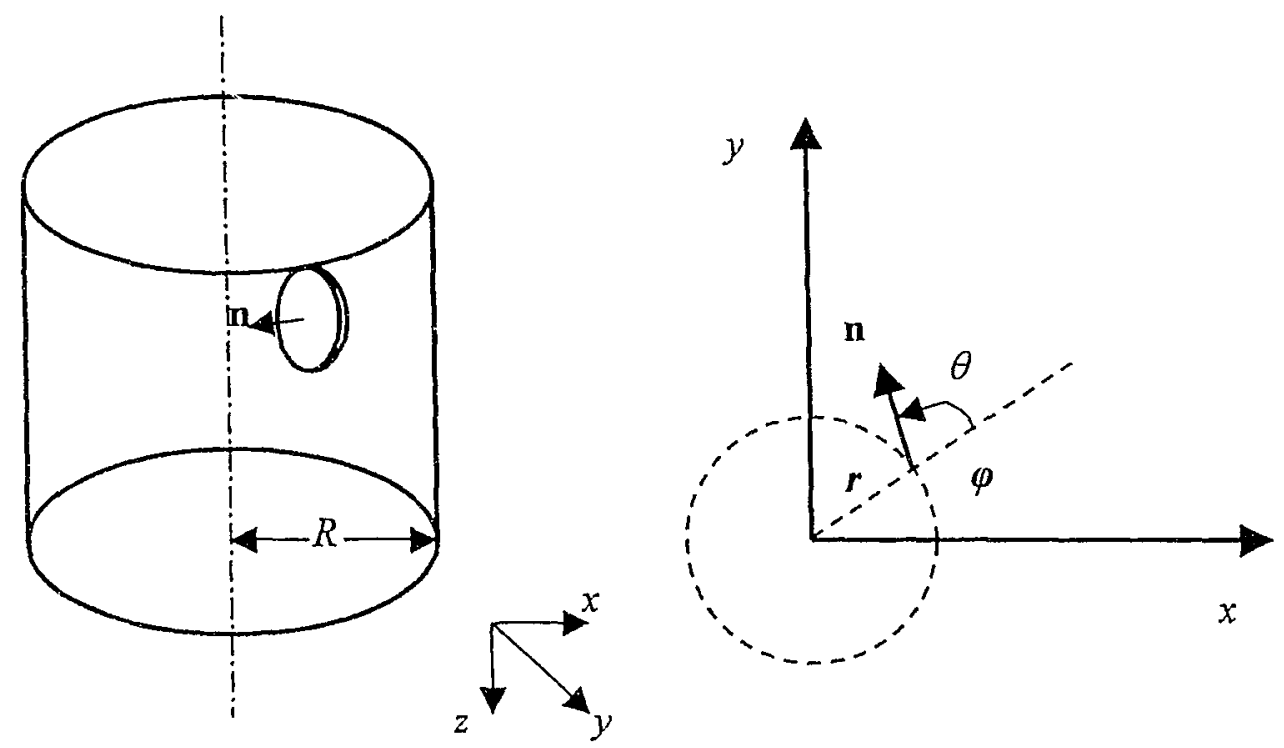

Figure 5.1 Schematic representation of the disc-like molecules within a section of a carbonaceous mesophase fiber of radius $R$, and the definition of the coordinate systems. The polar coordinates $r-\varphi$ are expressed by dashed lines. The Cartesian coordinates $x-y$ are expressed by solid lines. $\theta$ is the planar director orientation angle measured in radians, which is the angle between director $\mathbf{n}$ and polar $r$ at point $(x, y)$.

Since there are three unknowns, three balance equations are needed. The three equations that govern the behaviour of temperature $T$, director $\theta$, and the scalar order parameter $S$ are the energy balance equation of the system, the $z$-component internal angular momentum balance equation, and the scalar order parameter balance equation, respectively. 


\subsection{Balance Equation Formulation}

This section consists of the development of the two-dimensional partial differential equations that govern the time evolution of temperature, director, and the scalar order parameter within the carbonaceous mesophase fiber during the cooling process after it is pulled out of the spinneret. First, the Landau-de Gennes energy term in the Ericksen continuum theory is defined in the two-dimensional space. Then the balance equations for director and the scalar order parameter are derived according to the modified version of the Leslie-Ericksen continuum theory. Lastly, the set of partial differential equations is nondimensionalized.

\subsubsection{Landau-de Gennes Free Energy}

In the Ericksen continuum theory, the static energy term is defined by Landau-de Gennes energy density expressed by equation (2.15). The coefficients $A, B$, and $C$ in the Landaude Gennes free energy density (eqution (2.15)) are not known for a mesophase pitch. It is then convenient to replace the terms introduced by these coefficients in the free energy density by the Doi and Edwards [29] approximation expressed by equation (2.19). The Landau-de Gennes free energy density of the system then can be expressed as:

$$
\begin{aligned}
f_{\mathrm{L}} & =k_{B} \cup T\left[\frac{1}{2}\left(1-\frac{1}{3} U\right) S^{2}-\frac{1}{9} U S^{3}+\frac{1}{6} U S^{4}\right] \\
& +\frac{3}{4}\left(L_{1}+\frac{1}{6} L_{2}\right)\left[\left(\frac{\partial S}{\partial x}\right)^{2}+\left(\frac{\partial S}{\partial y}\right)^{2}\right] \\
& +\frac{3}{8} L_{2}\left[\cos ^{2} \theta\left(\frac{\partial S}{\partial x}\right)^{2}+\sin (2 \theta) \frac{\partial S}{\partial x} \frac{\partial S}{\partial y}+\sin ^{2} \theta\left(\frac{\partial S}{\partial y}\right)^{2}\right] \\
& +\frac{9}{4} S^{2}\left[\left(\frac{\partial \theta}{\partial x}\right)^{2}+\left(\frac{\partial \theta}{\partial y}\right)^{2}\right]-\frac{3}{8} L_{2} S \sin (2 \theta) \frac{\partial \theta}{\partial x} \frac{\partial S}{\partial x}+\frac{3}{8} L_{2} S \sin (2 \theta) \frac{\partial \theta}{\partial y} \frac{\partial S}{\partial y} \\
& +\frac{3}{4} L_{2} S\left(1+\cos ^{2} \theta\right) \frac{\partial \theta}{\partial y} \frac{\partial S}{\partial x}-\frac{3}{4} L_{2} S\left(1+\sin ^{2} \theta\right) \frac{\partial \theta}{\partial x} \frac{\partial S}{\partial y}
\end{aligned}
$$




\subsubsection{Energy Balance Equation}

The temperature variation is derived using the energy balance equation of the system (see equation (4.3)). The two-dimensional expression of this derivation is:

$$
\rho C_{\mathrm{p}} \frac{\partial T}{\partial t}=k_{\mathrm{th}}\left(\frac{\partial^{2} T}{\partial x^{2}}+\frac{\partial^{2} T}{\partial y^{2}}\right)
$$

\subsubsection{Angular Momentum and Scalar Order Parameter Balance Equations}

To derive the $z$-component of the internal angular momentum balance equation, the assumptions given above, and equations (2.7), (2.12), (2.15), (2.17a), (A.1i), and (A.1j) are used. The result of this derivation is:

$$
\begin{aligned}
-\gamma_{1}^{S} \frac{\partial \theta}{\partial t}= & -9 S\left(L_{1}+\frac{1}{2} L_{2}\right)\left(\frac{\partial \theta}{\partial x}+\frac{\partial \theta}{\partial y}\right)\left(\frac{\partial S}{\partial x}+\frac{\partial S}{\partial y}\right)-\frac{9}{2} S^{2}\left(L_{1}+\frac{1}{2} L_{2}\right)\left(\frac{\partial^{2} \theta}{\partial x^{2}}+\frac{\partial^{2} \theta}{\partial y^{2}}\right) \\
& -\frac{3}{4} L_{2}\left(1+\cos ^{2} \theta\right)\left(\frac{\partial S}{\partial x}\right)^{2}+\frac{3}{4} L_{2}\left(1+\sin ^{2} \theta\right)\left(\frac{\partial S}{\partial y}\right)^{2} \\
& +\frac{3}{4} L_{2} \cos (2 \theta) \frac{\partial S}{\partial x} \frac{\partial S}{\partial y}-\frac{3}{4} L_{2} S\left(1+\cos ^{2} \theta-\sin \theta \cos \theta\right) \frac{\partial^{2} S}{\partial x^{2}} \\
& +\frac{3}{4} L_{2} S\left(1+\sin ^{2} \theta-\sin \theta \cos \theta\right) \frac{\partial^{2} S}{\partial y^{2}}+\frac{3}{4} L_{2} S \sin (2 \theta)\left(\frac{\partial \theta}{\partial x}-\frac{\partial \theta}{\partial y}\right)\left(\frac{\partial S}{\partial x}-\frac{\partial S}{\partial y}\right)
\end{aligned}
$$

To derive the scalar order parameter balance equation, the assumptions given above, and equations $(2.12),(2.15),(2.17 \mathrm{~b}),(2.18),(\mathrm{A} .1 \mathrm{~g})$, and $(\mathrm{A} .1 \mathrm{~h})$ are used. The result of this derivation is: 


$$
\begin{aligned}
-\beta_{2}^{S} \frac{\partial S}{\partial t}= & k_{\mathrm{B}} v T\left[\left(1-\frac{T_{\mathrm{NI}}}{T}\right) S-\frac{T_{\mathrm{N}}}{T} S^{2}+2 \frac{T_{\mathrm{NI}}}{T} S^{3}\right] \\
& +\frac{9}{2} S\left(L_{1}+\frac{1}{2} L_{2}\right)\left[\left(\frac{\partial \theta}{\partial x}\right)^{2}+\left(\frac{\partial \theta}{\partial y}\right)^{2}\right]-\frac{3}{2}\left(L_{1}+\frac{1}{6} L_{2}\right)\left(\frac{\partial^{2} S}{\partial x^{2}}+\frac{\partial^{2} S}{\partial y^{2}}\right) \\
& +\frac{9}{4} L_{2}\left(\sin \theta \cos \theta+\sin ^{2} \theta\right) \frac{\partial \theta}{\partial x} \frac{\partial S}{\partial x}-\frac{9}{4} L_{2}\left(\sin \theta \cos \theta+\cos ^{2} \theta\right) \frac{\partial \theta}{\partial y} \frac{\partial S}{\partial y} \\
& -\frac{3}{4} L_{2}\left(\sin \theta \cos \theta+\cos ^{2} \theta\right) \frac{\partial^{2} S}{\partial x^{2}}-\frac{3}{4} L_{2}\left(\sin \theta \cos \theta+\sin ^{2} \theta\right) \frac{\partial^{2} S}{\partial y^{2}} \\
& +\frac{3}{4} L_{2} S\left(1+\sin \theta \cos \theta+\sin ^{2} \theta\right) \frac{\partial^{2} \theta}{\partial x^{2}}-\frac{3}{4} L_{2} S\left(1+\sin \theta \cos \theta+\cos ^{2} \theta\right) \frac{\partial^{2} \theta}{\partial y^{2}} \\
& +\frac{3}{4} L_{2} S[\sin (2 \theta)+\cos (2 \theta)]\left(\frac{\partial \theta}{\partial x}\right)^{2}+\frac{3}{4} L_{2} S[\sin (2 \theta)-\cos (2 \theta)]\left(\frac{\partial \theta}{\partial y}\right)^{2}
\end{aligned}
$$

Using relations derived by Beris and Edwards [26] (see equations (A.1a-n)), the final results of these derivations are, respectively, as follows:

$$
\begin{aligned}
-\gamma_{1} \frac{\partial \theta}{\partial t} & =\kappa_{10}\left(\frac{\partial \theta}{\partial x}+\frac{\partial \theta}{\partial y}\right)\left(\frac{\partial S}{\partial x}+\frac{\partial S}{\partial y}\right)+\kappa_{11}\left(\frac{\partial^{2} \theta}{\partial x^{2}}+\frac{\partial^{2} \theta}{\partial y^{2}}\right) \\
& +\kappa_{12} \frac{\partial S}{\partial x} \frac{\partial S}{\partial y}+\kappa_{13}\left(\frac{\partial S}{\partial x}\right)^{2}+\kappa_{14}\left(\frac{\partial S}{\partial y}\right)^{2} \\
& +\kappa_{15} \frac{\partial^{2} S}{\partial x^{2}}+\kappa_{16} \frac{\partial^{2} S}{\partial y^{2}} \\
& +\kappa_{17}\left(\frac{\partial \theta}{\partial x}-\frac{\partial \theta}{\partial y}\right)\left(\frac{\partial S}{\partial x}-\frac{\partial S}{\partial y}\right) \\
-\gamma_{1} \frac{\partial S}{\partial t} & =\kappa_{\mathrm{B}} v T\left[\left(1-\frac{T_{\mathrm{NI}}}{T}\right) S-\frac{T_{\mathrm{NI}}}{T} S^{2}+2 \frac{T_{\mathrm{NI}}}{T} S^{3}\right]\left(3+6 S^{2}\right) \\
& +\kappa_{18}\left[\left(\frac{\partial \theta}{\partial x}\right)^{2}+\left(\frac{\partial \theta}{\partial y}\right)^{2}\right]+\kappa_{19}\left(\frac{\partial^{2} S}{\partial x^{2}}+\frac{\partial^{2} S}{\partial y^{2}}\right) \\
& +\kappa_{20} \frac{\partial \theta}{\partial x} \frac{\partial S}{\partial x}+\kappa_{21} \frac{\partial \theta}{\partial y} \frac{\partial S}{\partial y}+\kappa_{22} \frac{\partial^{2} S}{\partial x^{2}}+\kappa_{23} \frac{\partial^{2} S}{\partial y^{2}} \\
& +\kappa_{24} \frac{\partial^{2} \theta}{\partial x^{2}}+\kappa_{25} \frac{\partial^{2} \theta}{\partial y^{2}}+\kappa_{26}\left(\frac{\partial \theta}{\partial x}\right)^{2}+\kappa_{27}\left(\frac{\partial \theta}{\partial y}\right)^{2}
\end{aligned}
$$


In equations (5.7) and (5.8) the elastic functions $\left\{\kappa_{i}\right\}, i=10, \ldots, 27$, are given in the Appendix. It is should be noticed that they are also dependency of director orientation angle $\theta$ and the scalar order parameters $S$.

\subsubsection{Dimensionless Form Equations}

The following scaling relations are used to nondimensionlize the resulting governing equations:

$T^{*}=\frac{T}{T_{\mathrm{NI}}} \quad$ (for dimensionless temperature)

$\kappa_{i}^{*}=\frac{\kappa_{i}}{K} \quad$ (for dimensionless elasticity)

$x^{*}=\frac{x}{R} \quad$ (for dimensionless space in $x$-direction)

$y^{*}=\frac{y}{R} \quad$ (for dimensionless space in $y$-direction)

$t^{*}=\frac{K}{\gamma_{1} R^{2}} t \quad$ (for dimensionless time)

By doing this, equations (5.4), (5.7) and (5.8) become the following dimensionless form:

$$
\frac{\partial T^{*}}{\partial t^{*}}=\alpha\left(\frac{\partial^{2} T^{*}}{\partial x^{* 2}}+\frac{\partial^{2} T^{*}}{\partial y^{* 2}}\right)
$$




$$
\begin{aligned}
-\frac{\partial \theta}{\partial t^{*}}= & \kappa_{10}^{*}\left(\frac{\partial \theta}{\partial x^{*}}+\frac{\partial \theta}{\partial y^{*}}\right)\left(\frac{\partial S}{\partial x^{*}}+\frac{\partial S}{\partial y^{*}}\right)+\kappa_{11}^{*}\left(\frac{\partial^{2} \theta}{\partial x^{* 2}}+\frac{\partial^{2} \theta}{\partial y^{* 2}}\right) \\
& +\kappa_{12}^{*} \frac{\partial S}{\partial x^{*}} \frac{\partial S}{\partial y^{*}}+\kappa_{13}^{*}\left(\frac{\partial S}{\partial x^{*}}\right)^{2}+\kappa_{14}^{*}\left(\frac{\partial S}{\partial y^{*}}\right)^{2} \\
& +\kappa_{15}^{*} \frac{\partial^{2} S}{\partial x^{* 2}}+\kappa_{16}^{*} \frac{\partial^{2} S}{\partial y^{* 2}}+\kappa_{17}^{*}\left(\frac{\partial \theta}{\partial x^{*}}-\frac{\partial \theta}{\partial y^{*}}\right)\left(\frac{\partial S}{\partial x^{*}}-\frac{\partial S}{\partial y^{*}}\right) \\
-\frac{\partial S}{\partial t^{*}} & =\beta\left[\left(T^{*}-1\right) S-S^{2}+2 S^{3}\right]\left(3+6 S^{2}\right) \\
& +\kappa_{18}^{*}\left[\left(\frac{\partial \theta}{\partial x^{*}}\right)^{2}+\left(\frac{\partial \theta}{\partial y^{*}}\right)^{2}\right]+\kappa_{19}^{*}\left(\frac{\partial^{2} S}{\partial x^{* 2}}+\frac{\partial^{2} S}{\partial y^{* 2}}\right) \\
& +\kappa_{20}^{*} \frac{\partial \theta}{\partial x^{*}} \frac{\partial S}{\partial x^{*}}+\kappa_{21}^{*} \frac{\partial \theta}{\partial y^{*}} \frac{\partial S}{\partial y^{*}}+\kappa_{22}^{*} \frac{\partial^{2} S}{\partial x^{* 2}}+\kappa_{23}^{*} \frac{\partial^{2} S}{\partial y^{* 2}} \\
& +\kappa_{24}^{*} \frac{\partial^{2} \theta}{\partial x^{* 2}}+\kappa_{25}^{*} \frac{\partial^{2} \theta}{\partial y^{* 2}}+\kappa_{26}^{*}\left(\frac{\partial \theta}{\partial x^{*}}\right)^{2}+\kappa_{27}^{*}\left(\frac{\partial \theta}{\partial y^{*}}\right)^{2}
\end{aligned}
$$

Equation (5.10) introduces a dimensionless thermal diffusivity $\alpha$ and a dimensionless characteristic molecular free energy $\beta$, which are expressed as follow:

$$
\begin{gathered}
\alpha=\frac{k_{\mathrm{th}}}{\rho C_{\mathrm{p}}} \frac{\gamma_{\mathrm{I}}}{K} \\
\beta=\frac{k_{\mathrm{B}} v T_{\mathrm{NI}} R^{2}}{K}
\end{gathered}
$$

As mentioned in Chapter 4 , the dimensionless thermal diffusivity $\alpha$ depends on both the melt transport properties and the liquid crystalline properties. $\beta$ is defined as the ratio of short-range order elasticity to long-range order elasticity [20], and in this study $\beta=10^{4}$ is used to be consistent with prior published theoretical work. To be consistent with the one-dimensional study, in this two-dimensional model, the same range $1 \leq \alpha \leq 10^{5}$ is used. 
The material physical properties and the melt spinning process parameters used in this chapter are also same as those that are used in Chapter 4 and are tabulated in Tables 4.1 to 4.3. The values for $K, K_{5}$, and $K_{6}$ are obtained by assuming the same ratio of $L_{2} / L_{1}$, as defined in Chapter 4, which is in the same range as published theoretical work [20-23]. The absolute values of the three elastic constants are of the order of $10^{-12} \mathrm{~N}$ [24]. Furthermore, the elastic constants satisfy the constitutive hypothesis set by the following conditions [34]:

$$
L_{1} \geq 0 \text { and } L_{1}+\frac{2}{3} L_{2} \geq 0
$$

Lastly, according to equation $(2.16 \mathrm{~b}, \mathrm{c}), L_{2}<0$ is required.

\subsection{Auxiliary Equations}

\subsubsection{Initial Conditions}

The initial molecular orientation are formed during melt spinning extrusion flow and aligned along the flow direction with some thermal fluctuation $[9,13,37]$. The fluctuation is assumed to be random, which generated by a build-in random number generator. Then the initial director field can be expressed as:

$\theta_{i}=\theta_{0}+\eta \varepsilon$

where $\theta_{0}$ represent director field initial average orientation angle, $\varepsilon$ is a random number determined using a standard random number generator and is within the range $0<\varepsilon<1$, and $\eta$ is a factor that controls the magnitude of the fluctuation. In this study, $\eta=0.5$ is used. The choice of the algebraic operation \pm is determined randomly by using a random number generator with a different seed than that used to generate $\varepsilon$. If the random number generated is less than 0.5 , the sign is negative; otherwise, it is positive. 
Initial temperatures take as the spinning temperature at everywhere of the fiber cross section, and the initial scalar order parameters are assumed as at the equilibrium value. According to above assumptions, the initial conditions can be expressed in the following equations:

$T_{i}=T_{\mathrm{S}}$ at $t=0,-R \leq x \leq R,-R \leq y \leq R$

$\theta_{i}=\theta_{\mathrm{o}}+\eta \varepsilon$ at $t=0,-R \leq x \leq R,-R \leq y \leq R$

$S_{i}=0.25+0.75 \sqrt{1-\frac{8 T_{\mathrm{S}}}{9 T_{\mathrm{M}}}}$ at $t=0,-R \leq x \leq R,-R \leq y \leq R$

\subsubsection{Boundary Conditions}

The realistic boundary conditions for molecular orientation used in this study represent two typical surface anchoring behaviours of mesophase pitch molecules during the fabrication of carbon fibers. One boundary condition represents the case where the discotic liquid crystalline molecules are oriented with the aromatic rings perpendicular to the spinneret. This is obtained by setting the directors, which are perpendicular to the discotic molecules at $\theta_{\mathrm{b}}=0$. Another possible boundary condition for the director field is where the discotic liquid crystalline molecules are aligned parallel (i.e., tangential) to the spinneret surface. This is represented in the model by setting the director orientation angles at the spinneret surface to be $90^{\circ}$, i.e., $\theta_{b}=\pi / 2$ radians.

Boundary conditions for temperature and the scalar order parameter fields are assumed as Dirichlet conditions. For temperature field, the surface of fiber takes the cooling air temperature; the scalar order parameters take the equilibrium value at the surface temperature.

The boundary conditions are expressed as follows: 
$T=T_{\mathrm{C}}$ at $t>0, x^{2}+y^{2}=R^{2}$

$\theta=\theta_{\mathrm{b}}$ at $t>0, x^{2}+y^{2}=R^{2}$

$S=0.25+0.75 \sqrt{1-\frac{8 T_{\mathrm{C}}}{9 T_{\mathrm{NI}}}}$ at $t>0, x^{2}+y^{2}=R^{2}$

Using the same scaling method defined in Section 5.2.4, the dimensionless initial and boundary conditions can be expressed as follows:

$T_{j}^{*}=T_{\mathrm{S}} / T_{\mathrm{NI}}$ at $t^{*}=0,-1 \leq x^{*} \leq 1,-1 \leq y^{*} \leq 1$

$\theta_{i}=\theta_{0}+\eta \varepsilon$ at $t^{*}=0,-1 \leq x^{*} \leq 1,-1 \leq y^{*} \leq 1$

$S_{i}=0.25+0.75 \sqrt{1-\frac{8 T S}{9 T_{N 1}}}$ at $t^{*}=0,-1 \leq x^{*} \leq 1,-1 \leq y^{*} \leq 1$

$T^{*}=T_{\mathrm{C}} / T_{\mathrm{NI}}$ at $t^{*}>0, x^{* 2}+y^{* 2}=1$

$\theta=\theta_{b}$ at $t^{*}>0, x^{* 2}+y^{* 2}=1$

$S=0.25+0.75 \sqrt{1-\frac{8 T_{\mathrm{C}}}{9 T_{\mathrm{N}}}}$ at $t^{*}>0, x^{* 2}+y^{* 2}=1$

In equations (5.15) and $(5.16), T_{\mathrm{S}}$ is the fiber spinning temperature, $T_{\mathrm{C}}$ is the cooling air temperature, and $T_{\mathrm{NI}}$ is the nematic-isotropic phase transition temperature. Values for these temperatures are listed in Table 4.2. 
The initial average angles and boundary director orientations $\theta_{0}$ and $\theta_{\mathrm{b}}$ used in the simulation for this model are listed in Table 5.1. These values enable us to achieve the objectives stated earlier.

Table 5.1 Auxiliary conditions

\begin{tabular}{|c|c|c|}
\hline & Case 1 & Case 2 \\
\hline $\begin{array}{c}\text { Initial director average orientations, } \\
\theta_{0}\end{array}$ & $\theta_{\mathrm{o}}=\frac{\pi}{2}$ (radians) & $\theta_{\mathrm{o}}=0$ (radians) \\
\hline $\begin{array}{c}\text { Boundary director orientations, } \\
\theta_{\mathrm{b}}\end{array}$ & $\theta_{\mathrm{b}}=\frac{\pi}{2}$ (radians) & $\theta_{\mathrm{b}}=0$ (radians) \\
\hline
\end{tabular}

\subsection{Method of Solutions}

The governing equations derived in this study are three coupled non-linear partial differential equations, and require numerical method for solution. The Galerkin finite element method (GFEM) and the first order Euler predictor-corrector time integrator are used in the study to solve the set of equations. Detailed description of the GFEM can be found elsewhere [53-55]. The GFEM are used to discrete the space to generate a set of non-linear ordinary differential equations. The integration was performed using the Gaussian Quadrature method. The set of equations are solved simultaneously with a Newton-Raphason iteration method. Convergence is assumed as the difference of the length of the solution vectors between two successive computed solution vectors is less than $10^{-6}$. A mesh of 300 quadrilateral elements with 321 nodes is used in this study. The source code is written using Fortran 77. The computational solutions are obtained by running the source code on a SunUltra 60 Workstation. The CPU time required for the two-dimensional model is about 6 days. 


\subsection{Results and Discussion}

The results and discussion are presented in the following parts. First it shows the representative results for the molecular orientations, which are plotted through simulations for the two cases using the initial and boundary conditions listed in Table 5.1. The followed presentation and discussion on the free energy, temperature and the scalar order parameter fields are typical and applicable to both cases in Table 5.1. In this chapter, the representative case chosen is when $\alpha=10^{3}$ and $\beta=10^{4}$. The section begins by showing the time evolution of the molecular field, and temperature and the scalar order parameter spatial profiles for $\alpha=10^{3}$ and $\beta=10^{4}$. This is followed by a discussion on the effect of the dimensionless thermal diffusivity $\alpha$ on the thermal relaxation phenomenon. This section concludes with a discussion on how the dimensionless thermal diffusivity $\alpha$ affects the order development and texture formation in carbon fibers.

\subsubsection{Mode Selection}

Figure 5.2 shows the two-dimensional relaxation phenomena of the molecular field when $\alpha=10^{3}$ and $\beta=10^{4}$ for case 1 (first column), and case 2 (second column) at the following dimensionless times $t^{*}$ : (a) 0.0 , (b) 0.01 , (c) 0.016 , (d) 0.05 . The short line segments represent the edges of the discotic molecules, and are obtained by noting that a director is normal to the disc-like molecule. The first columf shows that, under the initial and boundary conditions restricted by case 1 , the molecules go from a randomly aligned state to a quasi-onion state, and sisally evolving into a perfectly concentric (i.e., onion) texture. In contrast, the second column shows that, under the initial and boundary conditions restricted by case 2 , the molecules go from a randomly aligned state to a zigzagged state, and eventually evolving into a perfectly radial texture. This is consistent with the experimental observations reported in the literatures [12,13]. Matsumoto [12] reported that extrusion through a large diameter capillary could yield fibers with concentric textures. Hamada et al [13] formed onion fibers by stirring the pitch upstream. Wang 
and Ray [50] predicted that at low temperature the bend elastic constant $K_{3}<K_{1}$, leading to a bend mode deformation, causing the pitch molecules to orient radially in fibers cross section; at high temperature, $K_{3}>K_{1}$, yielding a concentric, onion arrangement of the molecules in fibers cross-section [50]. In this study, due to the assumption $K_{1}=K_{3}$, the model could not predict the elastic constant effect on the texture selection. Nevertheless, the results show that the texture selection is dependency of multivariable, such as the initial texture, surface-anchoring effect, and consistent with prior published theoretical work $[37,56]$.

\subsubsection{Structure Development}

Figure 5.2 also shows the fiber transverse structure development during the thermal relaxation period. It shows that the fiber textures evolve from stressed high-energy states to structures with minimal distortion Frank elastic free energy at steady state. The driving force for the structure development is the minimization of the stored Frank elastic free energy $F_{d}^{*}$, as shown in Figure 5.3.

In summary, the scientific visualizations of the fiber cross-sectional textures plotted from the computer simulations indicate that the mesophase pitch based carbon fiber texture formation is a combination of mode (radial or onion) selection and structure development. The mode selection between radial and onion textures depends on the mesophase pitch textures inherited from upstream and surface anchoring behaviours of mesophase pitch molecules. The structure development can be well explained using the Frank elastic free energy theory. Discotic nematics are elastic materials, where energy is stored by orientation strains. The driving force for the texture developing from a randomly aligned texture to a perfectly organized texture is the minimization of the stored Frank elastic free energy. 
(a)
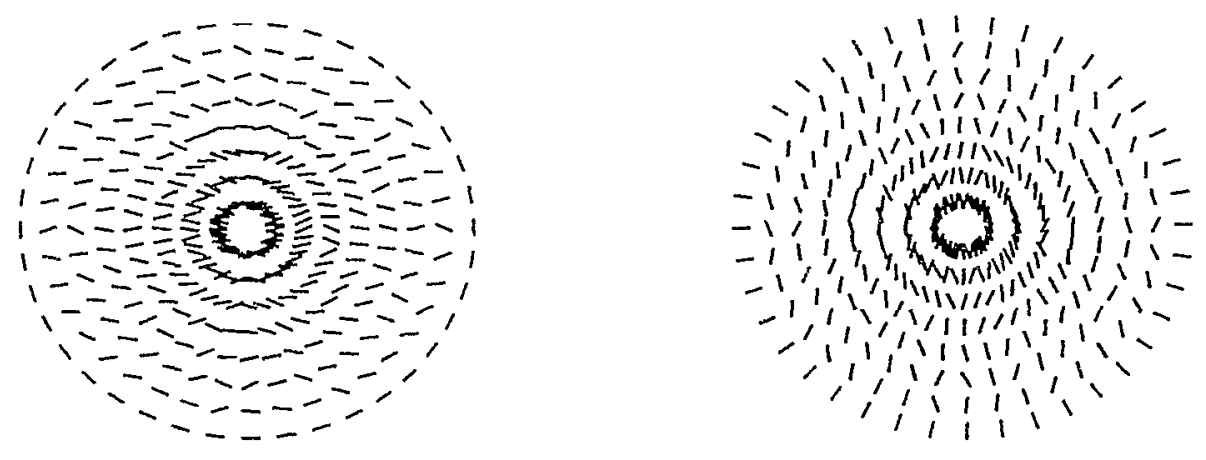

(b)
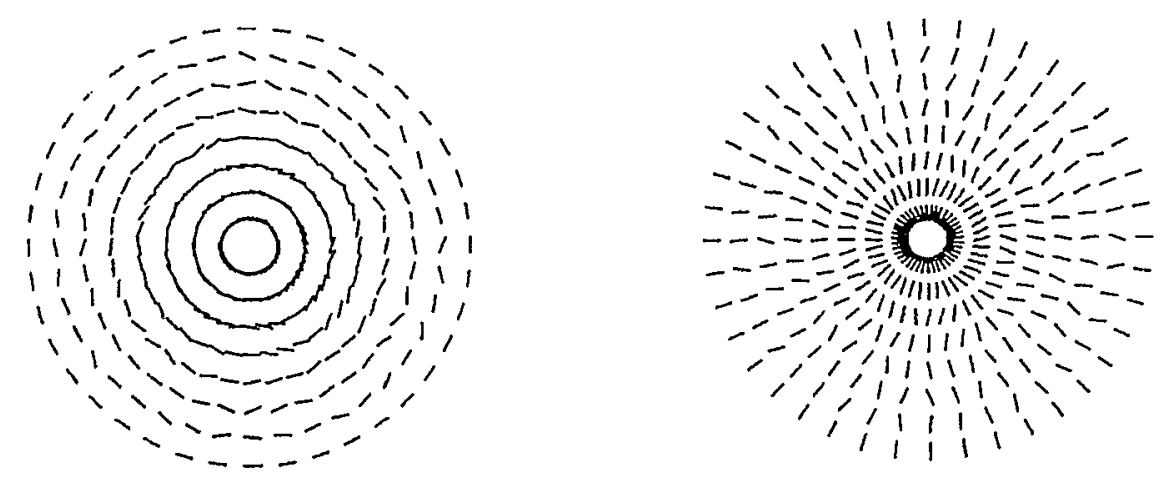

Figure 5.2 (continued on next page) Typical relaxation phenomena for molecular orientation for the case $\alpha=10^{3}$ and $\beta=10^{4}$, across fiber cross section at the following dimensionless times $t^{*}$ : (a) 0.0 , (b) 0.01 , (c) 0.016 , and (d) 0.05 . 
(c)
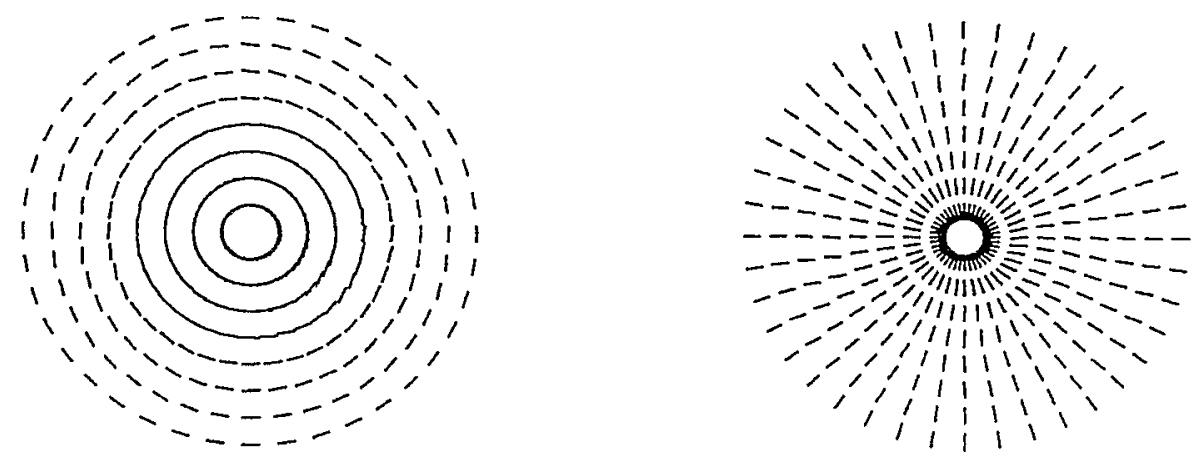

(d)
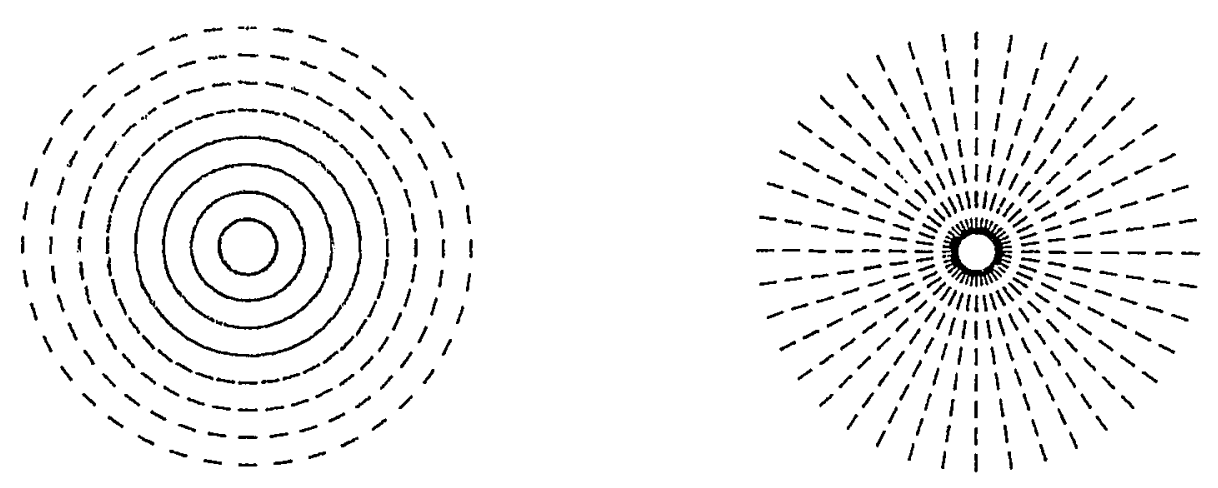

Figure 5.2 (continued) 


\subsubsection{Time Evolution of Frank Elastic Free Energy $F_{\mathrm{d}}{ }^{*}$}

Figure 5.3 is a plot of the stored Frank elastic free energy $F_{\mathrm{d}}{ }^{*}$ versus dimensionless time $t^{*}$. The two-dimensional dimensionless form $F_{\mathrm{d}}{ }^{*}$ may be obtained using equation (2.2) and is expressed as:

$$
F_{\mathrm{d}}^{*}=\frac{1}{4} \int_{-1}^{+1} \int_{-1}^{+1} S^{2}\left[\left(\frac{\partial \theta}{\partial x^{*}}\right)^{2}+\left(\frac{\partial \theta}{\partial y^{*}}\right)^{2}\right] d x^{*} d y^{*}
$$

In order to characterize the time evolution of the molecular reorientation from the totally random oriented state to the well-defined structure at steady state, $\tau_{\mathrm{d}}$ and $\tau_{\mathrm{o}}$ are defined (in the same way as in (hapter 4) as the time required for the molecules to reorient such that the Frank elastic free energy $F_{\mathrm{d}}{ }^{*}$ decreases by $86.5 \%$ and $98 \%$, respectively. For this case, $\tau_{\mathrm{d}}=0.016$ and $\tau_{\mathrm{o}}=0.05$. This figure shows that the Frank elastic free energy $F_{\mathrm{d}}{ }^{*}$ evolution goes through three stages: fast, intermediate, and weak relaxation. The three relaxation stages are consistent with the texture development exhibited in Figure 5.2. In the fast relaxation stage, corresponding to $t^{*}<0.016$ (or $\tau_{\mathrm{d}}$ ) in Figure 5.2, the molecules are initially randomly oriented which leads to a strained texture. This initial texture stores the greatest-level distortion free energy, which is the driving force for the fast relaxation rate for $t^{*}<\tau_{\mathrm{d}}$. In stage 2 , corresponding to 0.016 (or $\left.\tau_{\mathrm{d}}\right)<t^{*}<0.05$ (or $\tau_{0}$ ) in Figure 5.2, the Frank free energy $F_{\mathrm{d}}{ }^{*}$ decreases from $13.5 \%$ to $2 \%$ of its initial value; the molecules form well-defined structures at the end of this time period. In the final weak relaxation stage, corresponding to $t^{*}>0.05$ (or $\tau_{0}$ ) in Figure 5.2, the residue of the Frank elastic free energy $F_{d}{ }^{*}$ is the small driving force to reorient the molecules into the onion or radial textures. This is consistent with the time evolution of the Frank elastic free energy $F_{\mathrm{d}}{ }^{*}$ obtained from the one-dimensional model in Chapter 4. However, the total amount of the Frank elastic free energy $F_{d}{ }^{*}$ in this model is less than that of the onedimensional model calculated in Chapter 4, because this model used a smaller random number and generated a less randomly oriented initial structure. 


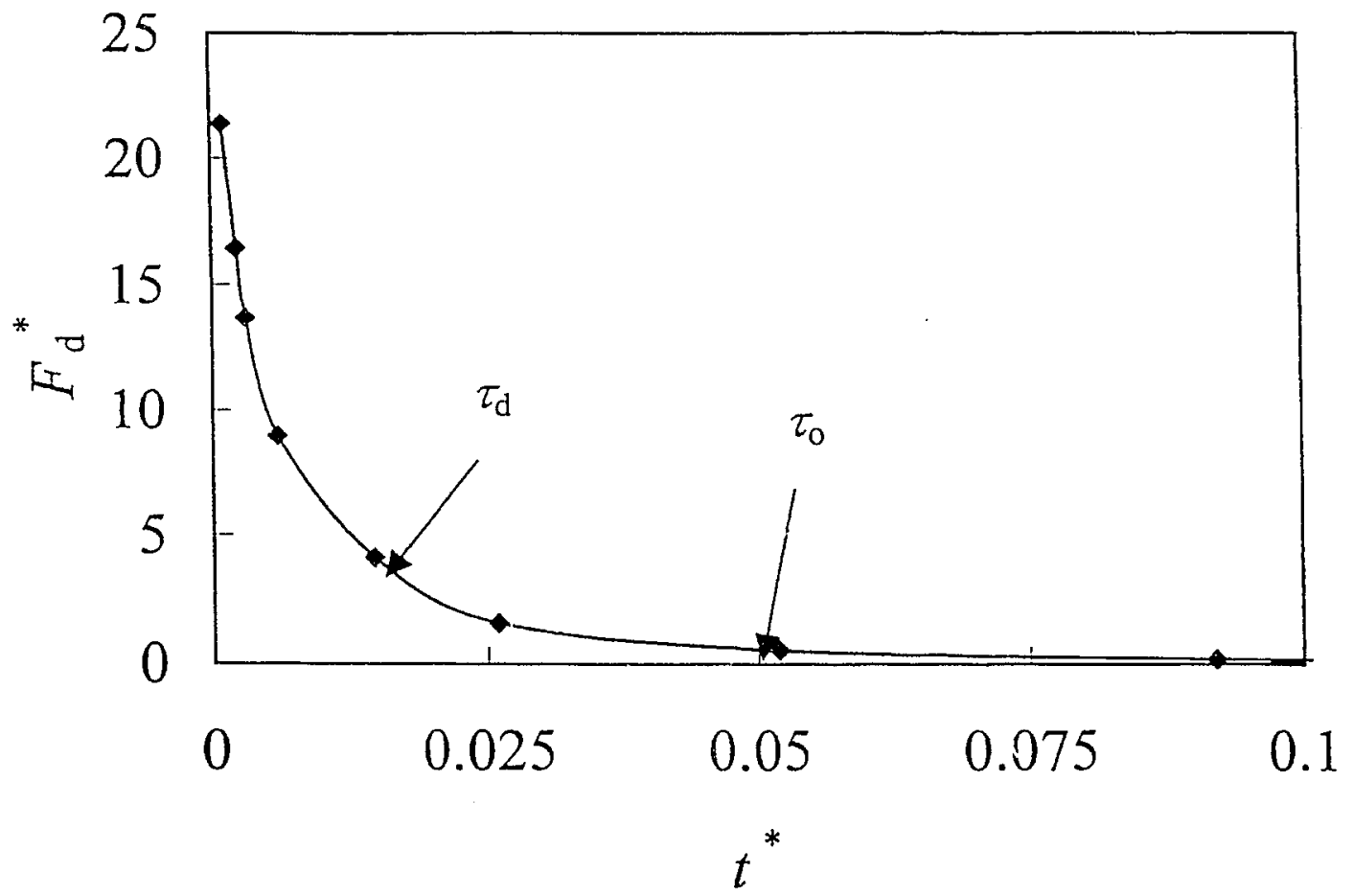

Figure 5.3 Tirne evolution of the dimensionless Frank elastic free energy $F_{\mathrm{d}}^{*}$ for the case $\alpha=10^{3}$ and $\beta=10^{4} . \tau_{\mathrm{d}}$ and $\tau_{\mathrm{o}}$ are defined as the time required for the molecules to reorient such that the Frank elastic free energy $F_{\mathrm{d}}{ }^{*}$ decreases by $86.5 \%$ and $98 \%$, respectively. 


\subsubsection{Temperature and The Scalar Order Parameter Profiles}

Figure 5.4 shows the two-dimensional relaxation phenomena of the temperature profile for the case when $\alpha=10^{3}$ and $\beta=10^{4}$ at the following dimensionless times $t^{*}$ : (a) 0.02 , (b) 0.03 , (c) 0.04 , and (d) 0.32 . Figure 5.4 shows the typical thermal diffusion phenomena across the fiber transverse section. The fiber exits the spinneret at spinning temperature $T_{\mathrm{S}}=600 \mathrm{~K}$, and is suddenly exposed to cooling air temperature of $T_{\mathrm{C}}=373$ $\mathrm{K}$. It is assumed that there is perfect thermal contact between the cooling air and the mesophase pitch. Therefore, when the two-dimensional temperature profile becomes uniform at steady state, the temperature of the entire fiber cross-section decreases to the cooling air temperature $T_{\mathrm{C}}$.

Figure 5.5 shows the two-dimensional relaxation phenomena of the scalar order parameter profiles for the case when $\alpha=10^{3}$ and $\beta=10^{4}$ at the following dimensionless times $t^{*}$ : (a) 0.02 , (b) 0.03 , (c) 0.04 , and (d) 0.32 . Figure 5.5 shows that the scalar order parameter $S$ evolution is consistent with that of the temperature profile. This is consistent with Doi and Edwards's approximation for the scalar order parameter at homogeneous equilibrium [35]:

$$
S_{\text {eq }}=0.25+0.75 \sqrt{1-\frac{8}{3 U}}
$$

where $U=3 T_{\mathrm{NI}} / T$. For an isotropic phase $U<8 / 3$, for a nematic phase $U>3$ [35]. For this model, nematic potential $U=3 T_{\mathrm{NI}} / T$ is inhomogeneous due to the temperature gradient across the fiber radius. The scalar order parameter $S$ is generally increasing reciprocally with temperature. The simulation results indicate that Doi and Edwards expression is a reasonable approximation of the scalar order parameter for a lowmolecular weight nematic phase with small fluctuation. 
(a)

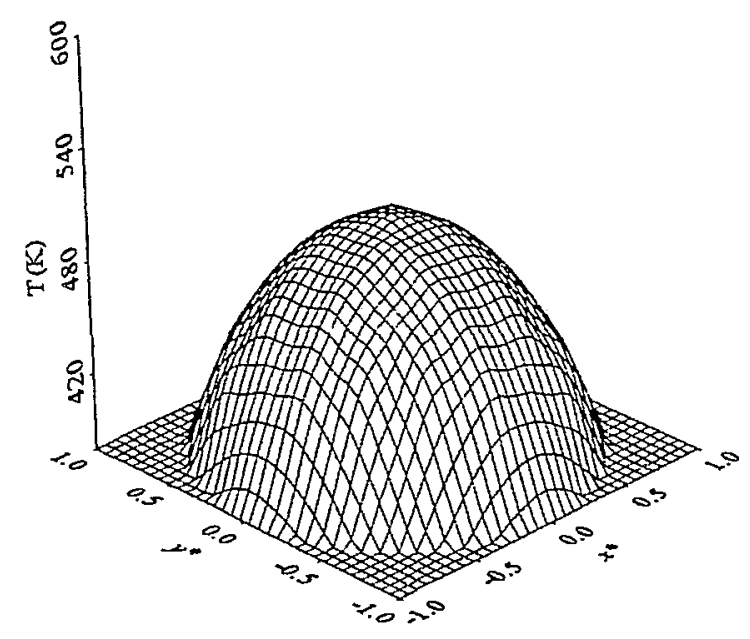

(b)

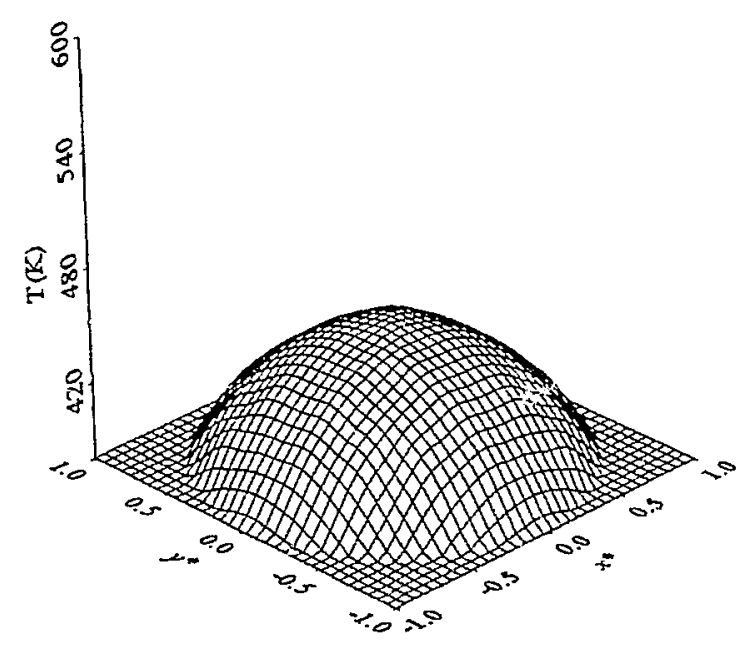

Figure 5.4 (continued on next page) Typical relaxation phenomena of the temperature profile for the case $\alpha=10^{3}$ and $\beta=10^{4}$ at the following dimensionless times $t^{*}$ : (a) 0.02 , (b) 0.03 , (c) 0.04 , and (d) 0.32 . 
(c)

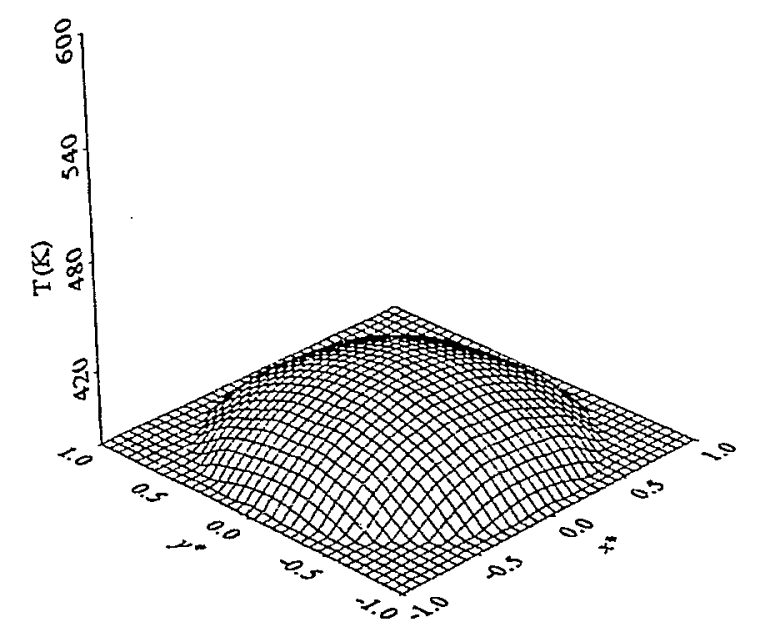

(d)

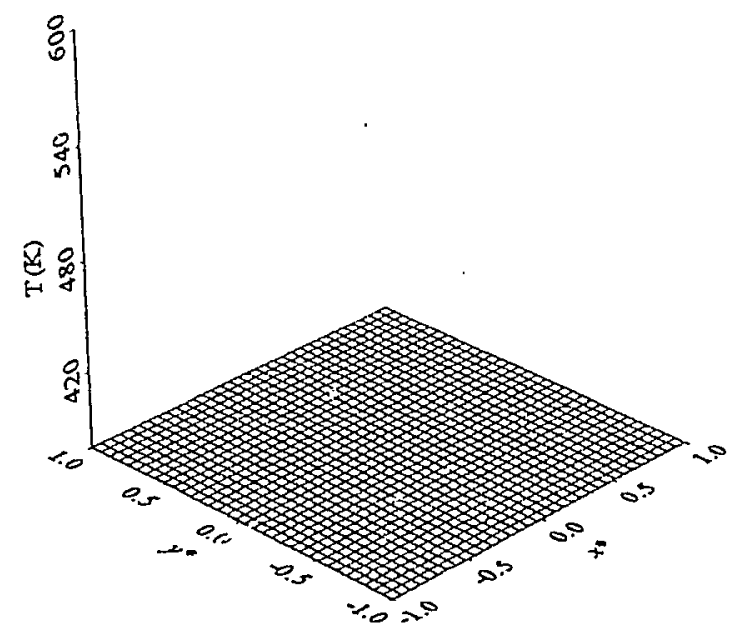

Figure 5.4 (continued) 
(a)

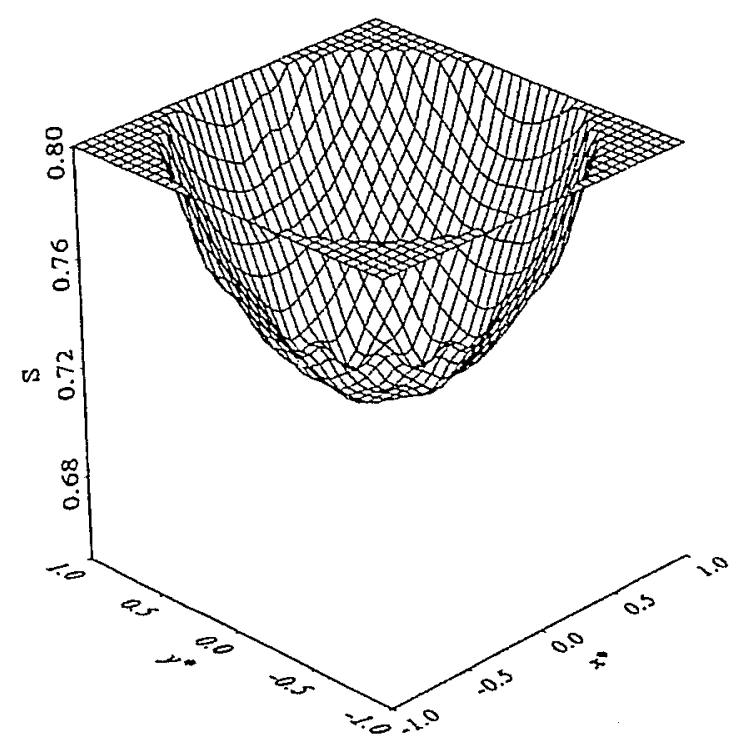

(b)

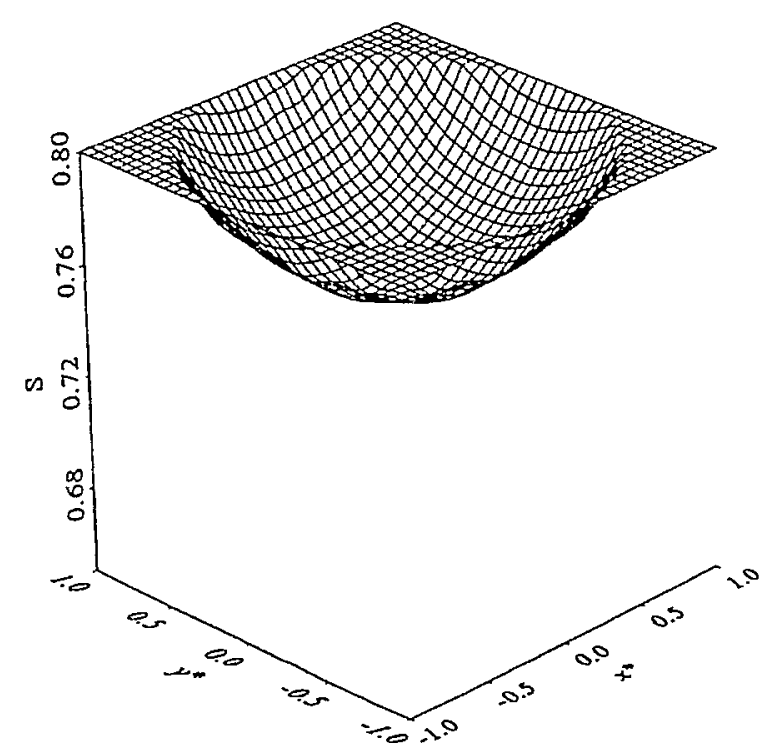

Figure 5.5 (continued on next page) Typical relaxatioti phenomena of the scalar order parameter profile for the case $\alpha=10^{3}$ and $\beta=10^{4}$ at the following dimensionless times $t^{*}$ : (a) 0.02 , (b) 0.03 , (c) 0.04 , and (d) 0.32 . 
(c)

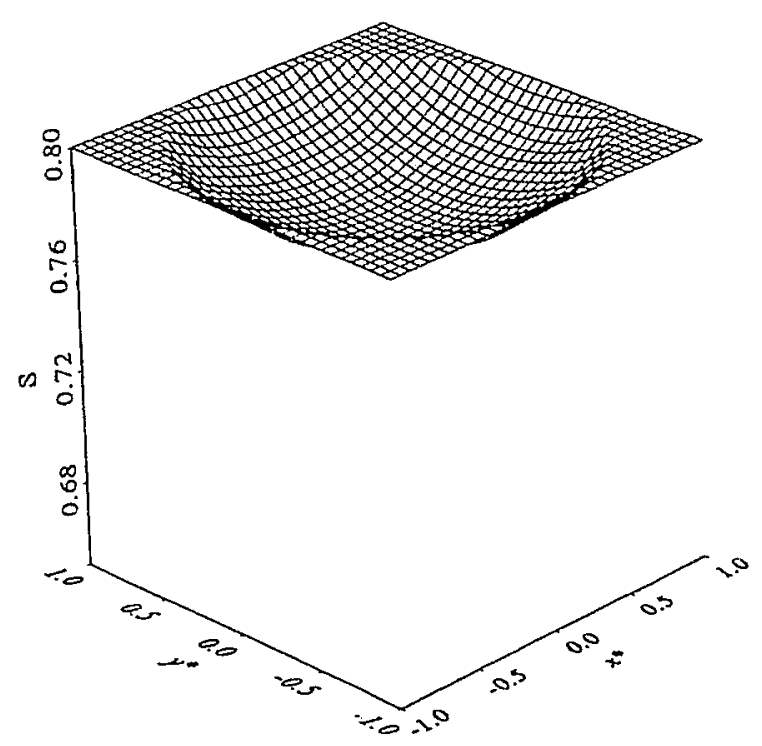

(d)

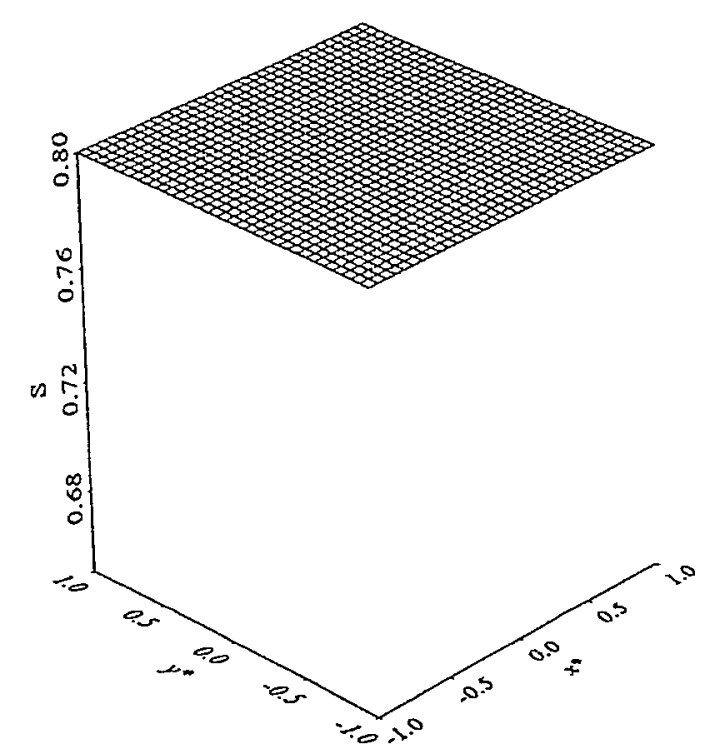

Figure 5.5 (continued)

Reproduced with permission of the copyright owner. Further reproduction prohibited without permission. 


\subsubsection{Time Evolution of Molecular Free Energy $F_{\mathrm{S}}{ }^{\star}$}

Figure 5.6 is a plot of the time evolution of the molecular free energy $F_{\mathrm{S}}{ }^{*}$ for the case when $\alpha=10^{3}$ and $\beta=10^{4}$, which was calculated by integrating equation (5.19) over the fiber cross-section area, and is expressed as:

$F_{S}^{*}=\beta \int_{-1}^{+1} \int_{-1}^{+1}\left[\frac{1}{2}\left(T^{*}-1\right) S^{2}-\frac{1}{3} U S^{3}+\frac{1}{2} U S^{4}\right] d x^{*} d y^{*}$

Figure 5.5 indicates that $F_{\mathrm{S}}{ }^{*}$ decreases with time, and reaches steady state at the same time as the spatial profiles of temperature and the scalar order parameter (see Figures 5.4 and 5.5). The time when $F_{\mathrm{S}}{ }^{*}$ decreases to the equilibrium value is denoted as the thermal relaxation process steady state time $\tau_{\alpha}$. For this case, the plot shows $\tau_{\alpha}=0.32$, and is consistent with the time required for temperature $T$ and the scalar order parameter $S$ reach steady state in Figures 5.4 and 5.5. The trend of the time evolution of the molecular free energy $F_{S}{ }^{*}$ for the two-dimensional model is found to be consistent with the simulation results of the one-dimensional model showed in Figure 4.5. However, for the same values of $\alpha$ and $\beta$, the specific time for $\tau_{\alpha}$ calculated from the two-dimensional model is much longer than that calculated from the one-dimensional model, and is closer to the thermal transport time reported in the literature [3]. 


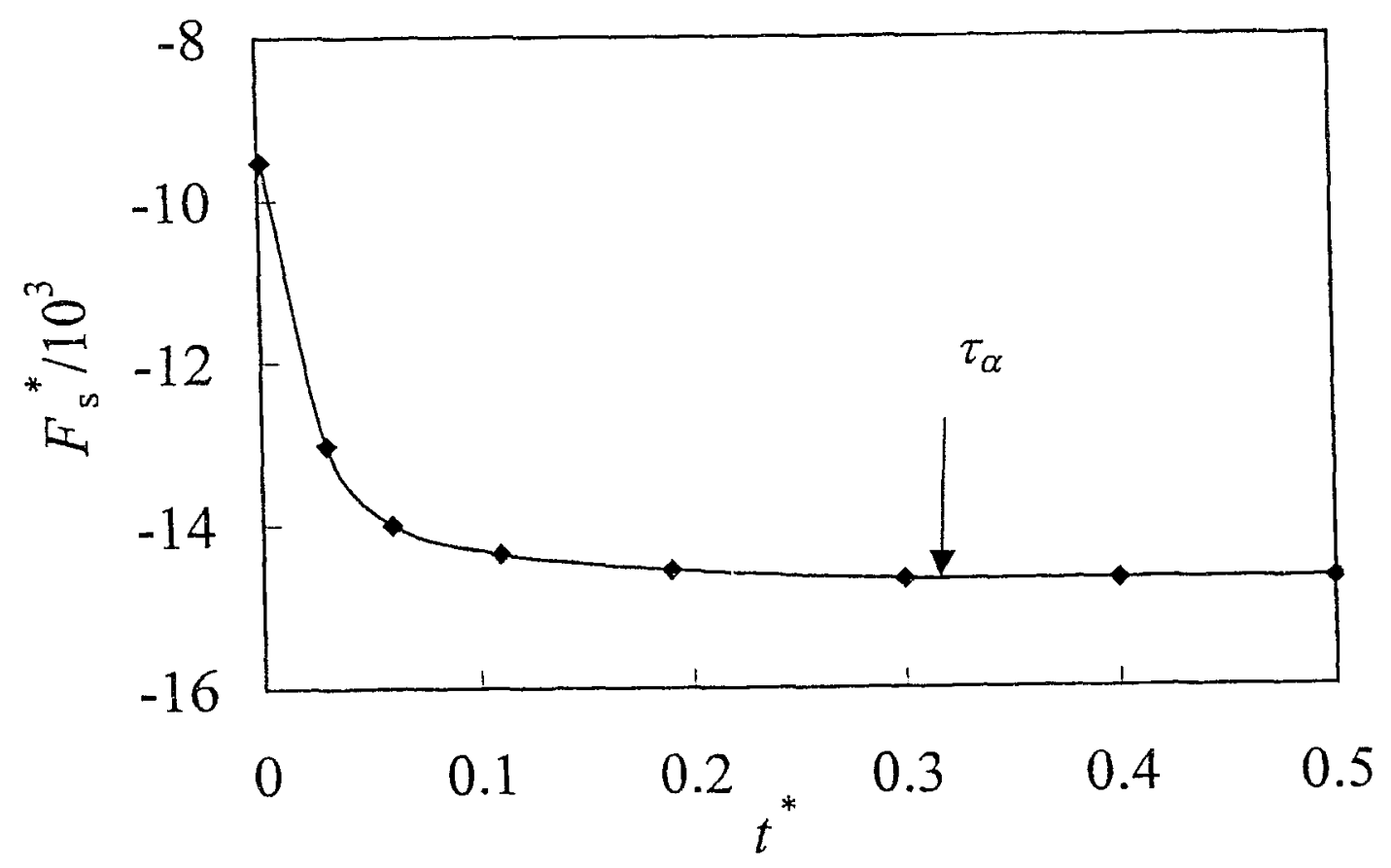

Figure 5.6 Time evolution of the dimensionless molecular free energy $F_{\mathrm{S}}^{*}$ for the case $\alpha=10^{3}$ and $\beta=10^{4} . \quad \tau_{\alpha}$ is the time for the molecular free energy to reach steady state. 


\subsubsection{Effect of $\alpha$ on The Thermal Relaxation}

This part study uses the same routine as that used in Chapter 4, the dimensionless process time scales $\tau_{\mathrm{d}}, \tau_{0}$ and $\tau_{\alpha}$ are simulated for different dimensionless thermal diffusivity $\alpha$ and plotted in Figure 5.7. This figure is used to discuss the effect of the dimensionless thermal diffusivity $\alpha$ on mesophase pitch based carbon fiber texture formation and ordered structure development. The slanted dashed line indicates that the thermal relaxation process steady state time $\tau_{\alpha}$ decreases monotonically with dimensionless thermal diffusivity $\alpha$. This means that the time for $S$ and $T$ to reach steady state decreases as $\alpha$ increases. The reason for this phenomenon is that the dimensionless thermal diffusivity is the factor that controls the rate of the heat transfer between the mesophase pitch and the cooling air (see equation (5.10a)), i.e., the rate of heat transfer increases with $\alpha$. The two solid near-horizontal lines represent the dependencies of the dimensionless director reorientation times $\tau_{\mathrm{o}}$ and $\tau_{\mathrm{d}}$ versus the dimensionless thermal diffusivity $\alpha$. The nearly flat lines show that the dimensionless thermal diffusivity $\alpha$ has little effect on the director field relaxation. These results are consistent with the onedimensional simulation conclusion, and also can be explained by the time evolution of molecular orientation equation. The dynamic behaviour of the director reorientation is governed by equation (5.10b). The right side of equation (5.10b) has eight terms. The first term, the second term and the last six terms are obtained, respectively, from $f_{\mathrm{e}}^{*}, f_{\mathrm{d}}^{*}$, and $f_{\mathrm{C}}{ }^{*}$. The simulation results indicated that, compared with Frank elastic free energy, $f_{\mathrm{e}}{ }^{*}$ and $f_{\mathrm{C}}{ }^{*}$ induced by interaction between $S$ and $\mathbf{n}$ spatial gradients are negligible. Therefore, the effect of the first term and the last six terms on the director reorientation in equation (5.10b) can be neglected. Consequently, the time evolution of the director field in two-dimensional space can be expressed as:

$$
-\frac{\partial \theta}{\partial t^{*}}=\kappa_{11}^{*}\left[\left(\frac{\partial^{2} \theta}{\partial x^{* 2}}\right)+\left(\frac{\partial^{2} \theta}{\partial y^{* 2}}\right)\right]
$$

Equation (5.20) shows that the relaxation of director field is not affected by the thermal 
diffusivity because the dimensionless elastic function $\kappa_{11}^{*}$ is related only to Landau elastic constants (see equation (A.3b)). The other two terms of equation (5.10b) have little effect on the director time evolution. This leads to the director reorientation behaviour being nearly independent of the scalar order parameter $S$ and temperature $T$ fields.

Using the same way as Chapter 4 , to characterize the thermal diffusivity effect on the texture formation and the ordered structure development, $\alpha_{0}$ is defined as the value of $\alpha$ at the space cross over point of $\tau_{\alpha}$ and $\tau_{\mathrm{o}}$, and the value at the cross over point between $\tau_{\alpha}$ and $\tau_{\mathrm{d}}$ is denoted as $\alpha_{\mathrm{d}}$. As discussed above, $\tau_{\alpha}$ may be taken as the time for the temperature $T$ and the scalar order parameter profile's spatial profiles to reach steady state. Furthermore, $\tau_{0}$ and $\tau_{\mathrm{d}}$ may be taken as the time for the liquid crystal molecule spatial profile to reorient to within $86.5 \%$ and $98 \%$, respectively, of its steady state profile. It should be noticed that this dimensionless thermal diffusivity $\alpha$ also cuunts in the ratio of viscosity and elastic properties expressed as $\gamma_{1} / K$. Consequently, high value of $\alpha$ indicates higher viscosity, lower elasticity, and fast cooling rate, low value of $\alpha$ represents lower viscosity, higher elasticity, and slow cooling process.

As Figure 5.7 shows that for $\alpha>\alpha_{\mathrm{d}}$, the process time $\tau_{\alpha}$ is less than reorientation times $\tau_{\mathrm{d}}$ and $\tau_{0}$. This means there is little time available for discotic molecules to rotate, leading a partially aligned texture depicted in Figure (5.2b). For $\alpha_{0}<\alpha<\alpha_{d}$, Figure 5.7 shows $\tau_{d}<\tau_{\alpha}<\tau_{0}$. When $\alpha$ is between this values, the molecules will have more time to reorient into the well-defined structures (i.e., radial or onion textures) exhibited in Figure (5.2c). For $\alpha<\alpha_{0}$, the plot shows $\tau_{\alpha}>\tau_{\mathrm{d}}$ and $\tau_{0}$. This means the reorientation time provided by the thermal relaxation process for molecules to rotate is longer than the time that the molecules require to reorient to well-defined radial or onion structures as exhibited in Figure (5.2d). 


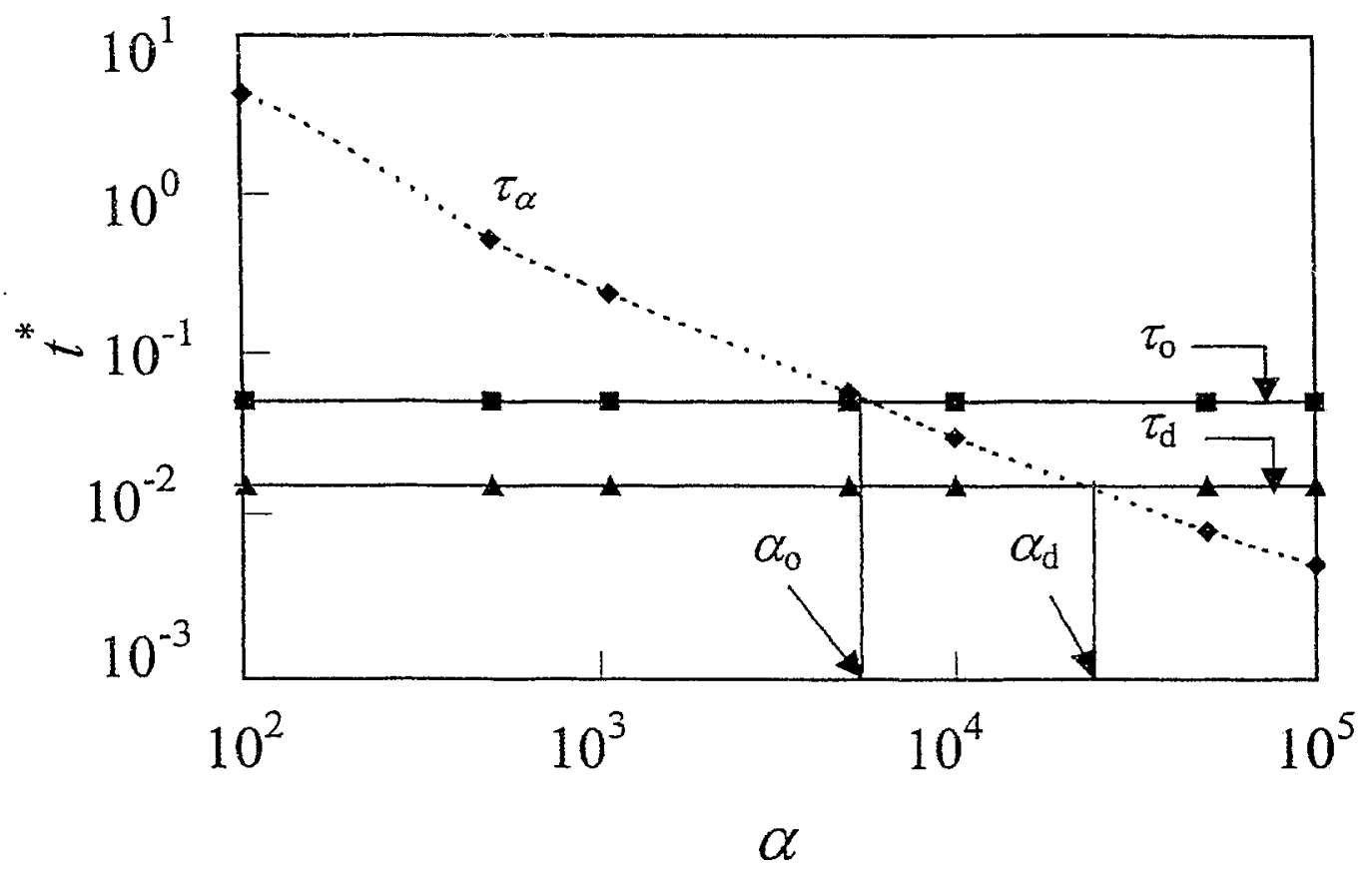

Figure 5.7 The effect of the thermal diffusivity $\alpha$ on the thermal relaxation. The dashed line represents the thermal relaxation time scale versus $\alpha$. The two solid lines represent the molecule reorientation time scales versus $\alpha . \alpha_{0}$ is defined as the cross point between $\tau_{\mathrm{o}}$ and $\tau_{\alpha}$, and $\alpha_{\mathrm{d}}$ is the cross point between $\tau_{\mathrm{d}}$ and $\tau_{\alpha}$. 


\subsection{Summary}

In this chapter, a two-dimensional model that describes the dynamic of mesophase pitch molecules in a nonisothermal system was developed and solved to study the structure development and texture formation in a discotic uniaxial nematic mesophase pitch based carbon fiber after cessation of extensional flow.

The simulation results are consistent with that obtained from the one-dimensional model. The structure development is affected by the cooling rate, pitch melt viscosity and elasticity. High thermal diffusivity caused by higher viscosity and fast cooling inhibits the liquid crystal molecular reorientation and limits the texture development. Lower viscosity and slow cooling provides more time for liquid crystal molecular reorientation and allows fiber cross sectional structures to develop into well-defined radial or onion textures.

Furthermore, the two-dimensional model is able of capturing five types of texture patterns, which match the commonly observed texture for mesophase pitch based carbon fibers. They are random, folded radial, radial, quasi-onion, and onion. These various textures represent the various combinations possible from the interplay between texture selection and structure development during the fiber spinning process. The mode selection between radial and onion textures are affected by the original texture and the surface anchoring behaviour of mesophase pitch molecules. The numerical results are consistent with production and experimental observations [12,13].

The two-dimensional model provides a better understanding of mesophase pitch based carbon fibers texture formation. The simulation results may provide additional information on a molecular orientation during melt spinning process and helps to decrease the post treatment temperature and to reduce the process cost. 


\section{Chapter 6}

\section{Conclusions and Recommendations for Future Work}

\subsection{Conclusions}

A mathematical model that describes the dynamic behavior of mesophase pitch molecules in a nonhomogenous temperature system has been developed, and solved in onedimensional and two-dimensional space, respectively. The simulation results from the one-dimensional and two-dimerisional models are consistent. The results from the present work proved that the mesophase pitch based carbon fiber texture formation is a combination of texture selection and structure development during cooling process. The textures obtained from the study are similar to that observed through experimental studies. They are: random, radial, zigzag radial, onion, and quasi-onion. Several important conclusions can be drawn from the understanding of this study:

(1) The results from the one-dimensional and two-dimensional models both indicate that the cooling step involves the relaxation of Frank elastic free energy $F_{\mathrm{d}}{ }^{*}$, taking into account director distortion. The transverse structure evolves from random to a welldeveloped texture (the radial or onion) to release the Frank elastic free energy $F_{\mathrm{d}}{ }^{*}$.

(2) High dimensionless thermal diffusivity caused by higher viscosity and fast cooling inhibits the liquid crystal molecular reorientation and limits the texture development. Lower viscosity and slow cooling provides more time for liquid crystal molecular reorientation and allows fiber cross sectional structure to develop into a well-ordered texture. 
(3) The results from the two-dimensional model shows that the mode selection between radial and onion textures are affected by the original texture and anchoring behaviors of mesophase pitch molecules. Wang and Rey's model indicated the stable texture depends on the relative size of the elastic constants $\left(K_{1}\right.$ and $\left.K_{3}\right)$ related with bend and splay deformation of the mesophase pitch. The Landau-de Gennes free energy used in this model assumes elastic constants $K_{1}$ and $K_{3}$ are equal, therefore the current model could not predict elastic constants effect on texture formation.

(4) Compared with one-dimensional model, the two-dimensional model gives better insight on the texture formation across mesophase pitch based carbon fibers, although the one-dimension model is capable of capturing the structure development for mesophase pitch based carbon fibers. Another advantage of the two-dimensional model is that it describes the transportation phenomena more accurately. The process time for heat transfer across the carbonaceous mesophase fiber cross section obtained from the twodimension model is much slower than that obtained from the one-dimensional model, and is closer to that of the actual thermal transportation in melt spinning process reported by the current literature [2]. In addition, the two-dimensional model has fewer assumptions and limitations, thus provided a better understanding on the structure development and texture formation ior mesophase pitch based carbon fibers.

\subsection{Contributions}

The viscoelastic model, compared to previous mathematical studies, contributes to the current texture formation of mesophase pitch based carbon fibers in the following aspects:

(1) The model takes into consideration of temperature gradients, which are present during the actual spinning fabrication process.

(2) The model demonstrates the structure development from random to well-defined textures. 
(3) The model is capable of predicting the process effect, such as the cooling rate, on texture formation.

(4) The elasticity and viscosity of mesophase pitch are both taken into account in the model.

(5) The effect of the liquid crystal molecular anchoring behaviour on the texture formation is also investigated in the model.

\subsection{Recommendations for Future Work}

Based on the findings presented in this work, the following recommendations are made for further study of the mesophase pitch carbon fiber texture formation:

(1) The simplifying assumptions that the material properties are constant in different directions should be removed.

(2) The effect of different shape geometry of the spinneret on the texture formation should be investigated.

(3) More realistic boundary conditions should be imposed. If the glass transition temperature $T_{\mathrm{g}}$ were incorporated in the boundary conditions, the results may be more realistic. 


\section{References}

1. Peebles, L.H., Carbon Fibers Formation, Structure, and Properties, London: CRC Press, 1995.

2. Edie, D.D., Carbon, 36, 345 (1997).

3. Donnet, J.B., Bansal, R., Carbon Fibers, New York: Marcel Dekker, 1990.

4. Edie, D.D., and Stoner, E.G., Carbon-Carbon Materials and Composites, edited by Buckley, J.D. and Edie, D.D., Noyes Publications, P41, 1992.

5. Lubin G., Handbook of Composites, New York: Van Nostrand Reinhold Company, 1981.

6. Fitz, J.D., Pennock, G.M., and Taylor, G.H., Carbon, 29, 1139 (1991).

7. Mochida, I., Yoon, S.H., and Takano, N., 1996, Carbon, 34, 941 (1996).

8. Mochida, I., Carbon, 38, 305 (2000).

9. McHugh, J.J., and Edie, D.D., Liquid Crystals, 18, 327 (1995).

10. Mochida, I., and Korai, Y., Fuel Soc. Japan, 64, 796 (1985).

11. Ogale, A.A., Lin, C., and Anderson, D.P., Carbon, 40,1309 (2002).

12. Matsumoto, T., and Pure Appl. Chem., 57,1553 (1985).

13. Hamada, T., Nishida, T.,Furuyama, M., and Tomika, T., Carbon, 26, 837 (1988).

14. White, J.L. and Buechler, M., Microstructure Formation in Mesophase Carbon Fibers and Other Graphitic Materials. In Petroleum-Derived Carbons, Edited by, Bacha, J.D., Newman, J.W., and White, J.L., ACS, Washington, pp.62-84, (1986).

15. Gallego, N.C., and Edie, D.D., Composites, Part A (32), 1031 (2001).

16. Edie, D.D., Robinson, K.E., Filerot, O., and Jones, S.P., Carbon, 32, 1045 (1994).

17. Sheng, P. and Prestley, E.B., and Wojtowicz, P.J., Introduction to Liquid Crystals, edited by Prestley, E.P., Wojtowicz, P.J., and Sheng, P., New York: Plenum Press, 1974. 
18. I-C. Khoo, and Simoni, F., Physics of Liquid Crystalline Materials, edited by I-C. Khoo, and Simoni, F., New York: Science Publishers, 1991.

19. de Gennes, P.G., The Physics of Liquid Crystals, Oxford: Clarendon Press, 1974.

20. Chandrasekhar, S., Liquid Crystals, $2^{\text {nd }}$ edition, Cambridge: Cambridge University Press, 1992.

21. de Jeu, W.H., Thermotropic Liquid Crystals, Fundamentals, New York: Springer-Verlag, 1988.

22. de Jeu, W.H., Physical Properties of Liquid Crystalline Materials, New York: Science publishers, 1980.

23. Oseen, C.W., Trans. Faraday Soc.,29, 833 (1933).

24. Zocher, H., Discuss. Faraday Soc.,29, 945 (1933).

25. Frank, R.C., Arch. Rat.Mech. Anal., 23, 266 (1966).

26. Beris, A.N., and Edwards, B.J., Thermodynamics of Flowing Systems, Oxford: Claredon, 1994.

27. Chan, P.K., and Rey, A.D., Liquid Crystals, 13, 775 (1993).

28. Ericksen, J.L., IMA Preprint Series No. 559 (1989)

29. Doi, M., Edwards, B.J., The theory of Polymer Dynamics, Oxford: Clarendon, 1986.

30. Brooks, J.D., and Taylor, G.H., Carbon, 3,185 (1965).

31. Mochida, I., Yoon, S-Ho., and Korai, Y., The Chemical Record, 2, 81 (2002).

32. Nishizawa, T., and Sakata, M., Fuel, 70,124 (1991).

33. Hoover, D., Davis, A., and Perotta, A.J., in Extended Abstracts Biennial conference on Carbon, 393 (1979).

34. Nazem, F.F., Fuel, 59, 851 (1980).

35. Singer, L.S., Carbon, 16, 408 (1978).

36. Edie, D.D. and Dunham, M.G., Carbon, 27, 647 (1989).

37. McHugh, J.J., and Edie, D.D., Carbon, 34, 1315 (1996). 
38. Hong, S.H., Korai,Y., and Mochida,I., Carbon 37, 917 (1999)

39. Fortin, F., and Yoon, S-Ho., Carbon, 32, 1119 (1994).

40. Robinson, K.E., and Edie, D.D., Carbon, 34, 13 (1996).

41. Bright, A.A., and Singer, L.S., Carbon, 17, 599 (1979).

42. Singh, A.P., and Rey, A.D. Liquid Crystals, 26, 825 (1999).

43. Singh, A.P., and Rey, A.D. Rheol. Acta, 37, 30 (1998).

44. Singh, A.P., and Rey, A.D. Rheol. Acta, 37, 374 (1998).

45. Singh, A.P., and Rey, A.D. Liquid Crystals, 18, 219 (1995).

46. Singh, A.P., and Rey, A.D., J. Non-Newtonian Fluid Mech., 94, 87 (2000).

47. Yan, J., and Rey, A.D., Carbon, 40, 2647 (2002).

48. de Andrada L.R.P., and Rey, A.D., J. Non-Newtonian Fluid Mech., 119, 71 (2004).

49. Sharma, D., and Rey, A.D., Liquid Crystals, 30, 377 (2003).

50. Wang, L., and Rey, A.D., Modeling Simulation in Material Science Engineering, 5, 67 (1997).

51. Bird, R.B., Stewart, W.E., and Lightfoot, E.N., Transport Phenomena, New York: Wiley, 1960.

52. Becker, E.B., Carey, G.F., Oden, J.T., Finite Elements: An Introduction, New Jersey: Prentice-Hall Inc., Englewood Cliffs, 1981.

53. Carey, G.F., and Oden, J.T., Finite Elements: A Second Course, New Jersey: Prentice-Hall Inc., Englewood Cliffs, 1983.

54. Reddy, J.N., An Introduction to the Finite Element Method, $2^{\text {nd }}$, New York: McGraw-Hill, 1993.

55. Akin, J.E., Application and Implementation of Finite Element Methods, New York: Academic Fress; London, 1984.

56. Wang, L., and Rey, A.D., Liquid Crystals, 23, 93 (1997). 


\section{Appendix}

The relations for the dependency of the Leslie viscosities on the scalar order parameter $S$ are as follows [26]:

$$
\begin{aligned}
& \alpha_{1}^{S}=\beta_{1}^{m} S^{2}-\frac{\left(\gamma_{2}^{S}\right)^{2}}{\gamma_{1}^{S}}+\frac{\left(\beta_{1}^{S}\right)^{2}}{\beta_{2}^{S}} \\
& \alpha_{2}^{S}=\frac{1}{2}\left(\gamma_{2}^{S}-\gamma_{1}^{S}\right) \\
& \alpha_{3}^{S}=\frac{1}{2}\left(\gamma_{2}^{S}+\gamma_{1}^{S}\right) \\
& \alpha_{4}^{S}=\beta_{4}^{m}+\frac{2}{3} \beta_{2}^{S}(1-S)+\frac{2}{9} \beta_{3}^{m}(1-S)^{2}+\frac{1}{9} \beta_{1}^{m}(1-S)^{2} \\
& \alpha_{5}^{S}=\frac{1}{3} \beta_{1}^{m} S(1-S)+\beta_{2}^{m} S+\alpha_{2}^{S} \frac{\gamma_{2}^{S}}{\gamma_{1}^{S}} \\
& \alpha_{6}^{S}=\gamma_{2}^{S}+\alpha_{5}^{S} \\
& \beta_{1}^{S}=\beta_{2}^{S} \frac{\alpha_{3}+\alpha_{2}}{\alpha_{3}-\alpha_{2}}(2 S+1)(1-S) \\
& \beta_{2}^{S}=\frac{\alpha_{3}-\alpha_{2}}{3+6 S^{2}} \\
& \gamma_{1}^{S}=S^{2}\left(\alpha_{3}-\alpha_{2}\right) \\
& \left(\alpha_{3}-\alpha_{2}\right) \frac{\left(\alpha_{3}+\alpha_{2}\right)}{3 S}+S \\
& \frac{1}{3}{ }^{2}
\end{aligned}
$$


where

$$
\begin{aligned}
& \beta_{1}^{m}=\alpha_{1}+\alpha_{2}-\alpha_{3}+4 \frac{\alpha_{2} \alpha_{3}}{\alpha_{2}-\alpha_{3}} \\
& \beta_{2}^{m}=\frac{1}{2} \alpha_{2} \\
& \beta_{3}^{m}=\frac{1}{2}\left(\alpha_{2}+\alpha_{5}\right)+2 \frac{\alpha_{2} \alpha_{3}}{\alpha_{2}-\alpha_{3}} \\
& \beta_{4}^{m}=\alpha_{4}
\end{aligned}
$$

The elastic functions $\left\{\kappa_{i}\right\}, i=1, \ldots, 9$, in equations (4.10b,c) are defined as follows:

$$
\begin{aligned}
& \kappa_{1}=-\frac{K}{S} \\
& \kappa_{2}=-\frac{1}{2} K \\
& \kappa_{3}=\frac{1}{2 S} K_{6} \sin (2 \theta) \\
& \kappa_{4}=\frac{1}{2} K S\left(3+6 S^{2}\right) \\
& \kappa_{5}=-K_{5}\left(3+6 S^{2}\right) \\
& \kappa_{6}=K_{6}\left(3+6 S^{2}\right) \sin (2 \theta) \\
& \kappa_{7}=-K_{6}\left(3+6 S^{2}\right) \cos ^{2} \theta
\end{aligned}
$$




$$
\begin{aligned}
& \kappa_{8}=K_{6} S\left(3+6 S^{2}\right) \cos (2 \theta) \\
& \kappa_{9}=\frac{1}{2} K_{6} S\left(3+6 S^{2}\right) \sin (2 \theta)
\end{aligned}
$$

The elastic functions $\left\{\kappa_{\mathrm{i}}\right\}, \mathrm{i}=10, \ldots, 27$ in equations $(5.10 \mathrm{~b}, \mathrm{c})$ are defined as follows:

$$
\begin{aligned}
& \kappa_{10}=-\frac{K}{S} \\
& \kappa_{11}=-\frac{1}{2} K \\
& \kappa_{12}=-\frac{1}{S^{2}} K_{6}\left(1+\cos ^{2} \theta\right) \\
& \kappa_{13}=\frac{1}{S^{2}} K_{6}\left(1+\sin ^{2} \theta\right) \\
& \kappa_{14}=\frac{1}{S^{2}} K_{6} \cos (2 \theta) \\
& \kappa_{15}=-\frac{1}{S} K_{6}\left(1+\cos ^{2} \theta-\sin \theta \cos \theta\right) \\
& \kappa_{16}=\frac{1}{S} K_{6}\left(1+\sin ^{2} \theta-\sin \theta \cos \theta\right) \\
& \kappa_{17}=\frac{1}{S} K_{6} \sin (2 \theta) \\
& \kappa_{18}=\frac{1}{2} K S\left(3+6 S^{2}\right)
\end{aligned}
$$




$$
\begin{aligned}
& \kappa_{19}=-K_{5}\left(3+6 S^{2}\right) \\
& \kappa_{20}=3 K_{6}\left(3+6 S^{2}\right)\left(\sin \theta \cos \theta+\sin ^{2} \theta\right) \\
& \kappa_{21}=-3 K_{6}\left(3+6 S^{2}\right)\left(\sin \theta \cos \theta+\cos ^{2} \theta\right) \\
& \kappa_{22}=-K_{6}\left(3+6 S^{2}\right)\left(\sin \theta \cos \theta+\cos ^{2} \theta\right) \\
& \kappa_{23}=-K_{6}\left(3+6 S^{2}\right)\left(\sin \theta \cos \theta+\sin ^{2} \theta\right) \\
& \kappa_{24}=K_{6} S\left(3+6 S^{2}\right)\left(1+\sin \theta \cos \theta+\sin ^{2} \theta\right) \\
& \kappa_{25}=-K_{6} S\left(3+6 S^{2}\right)\left(1+\sin \theta \cos \theta+\cos ^{2} \theta\right) \\
& \kappa_{26}=K_{6} S\left(3+6 S^{2}\right)[\sin (2 \theta)+\cos (2 \theta)] \\
& \kappa_{27}=K_{6} S\left(3+6 S^{2}\right)[\sin (2 \theta)-\cos (2 \theta)]
\end{aligned}
$$

In equations (A.2) and (A.3) the relations:

$$
\begin{aligned}
& K=9 L_{1}+\frac{9}{2} L_{2} \\
& K_{5}=\frac{3}{2} L_{1}+\frac{1}{4} L_{2}
\end{aligned}
$$




$$
K_{6}=\frac{3}{4} L_{2}
$$

have been used. 